Florida International University

FIU Digital Commons

\title{
The Experiences of Underrepresented Faculty and Administrators: A Study of Cultural Taxation
}

Katrina N. Amie

kamie001@fiu.edu

Follow this and additional works at: https://digitalcommons.fiu.edu/etd

Part of the Higher Education Commons

\section{Recommended Citation}

Amie, Katrina N., "The Experiences of Underrepresented Faculty and Administrators: A Study of Cultural Taxation" (2020). FIU Electronic Theses and Dissertations. 4407.

https://digitalcommons.fiu.edu/etd/4407

This work is brought to you for free and open access by the University Graduate School at FIU Digital Commons. It has been accepted for inclusion in FIU Electronic Theses and Dissertations by an authorized administrator of FIU Digital Commons. For more information, please contact dcc@fiu.edu. 


\section{FLORIDA INTERNATIONAL UNIVERSITY \\ Miami, Florida}

A dissertation submitted in partial fulfillment of the requirements for the degree of DOCTOR OF PHILOSOPHY in HIGHER EDUCATION

by

Katrina Ngozi Amie 
To: Dean Michael R. Heithaus

College of Arts, Sciences and Education

This dissertation, written by Katrina Ngozi Amie, and entitled The Experiences of Underrepresented Faculty and Administrators: A Study of Cultural Taxation, having been approved in respect to style and intellectual content, is referred to you for judgment.

We have read this dissertation and recommend that it be approved.

$\begin{array}{r}\hline \text { James Burns } \\ \hline \text { Maria Lovett } \\ \hline \text { Denjamin Baez, Major Professor }\end{array}$

Date of Defense: March 24, 2020

The dissertation of Katrina Ngozi Amie is approved.

Dean Michael R. Heithaus

College of Arts, Sciences and Education

Andrés G. Gil

Vice President for Research and Economic Development and Dean of the University Graduate School

Florida International University, 2020 


\section{DEDICATION}

I dedicate this dissertation to the two people who have dedicated their all to me: My parents. As a first-generation college graduate, this degree is for my parents and I, thus I personally hereby acknowledge them as honorary Dr. Shirley Amie and honorary Dr. Chukwuyem Amie for all of their hard work, support, motivation, and unconditional love from birth to this very moment in my life. It is their labor that created the foundation for my success. Thank you.

I also like to dedicate this dissertation to the little girl from the heart of Brownsville that believed she could and persisted despite the odds against her — that little girl is me. 


\section{ACKNOWLEDGMENTS}

I want to start by expressing my gratitude to my amazing committee, Dr.

Benjamin Baez, Dr. James Burns, Dr. Maria Lovett, and Dr. Daniel Saunders. Thank you all for your support, constructive feedback, time, and energy throughout this process.

Thank you for challenging me and molding me into the academic I am today. Each of you provided a unique contribution to my study and development as a researcher. I could not have asked for a better committee.

I want to give a special heartfelt thanks to Dr. Benjamin Baez, the absolute best major professor in the world, for guiding me throughout this journey- I never felt confused, astray, or discouraged. During my time in the higher education doctoral program, I often found myself humorously reflecting on how thankful I am to have met Dr. Baez so late in my academic career because, quite frankly, he sets a standard that is tough to follow in the academic world; Meeting him any earlier would have possibly negatively influenced my perception of my academic journey with others (Although I would have been fortunate to have met him sooner). He is one of the most authentic, caring, and supportive professors I have ever encountered. He is truly a gift to the Higher Education program and Florida International University. I came into this program anxious and unsure of myself at times - I'll attribute that to occasionally falling victim to imposter syndrome; however, Dr. Baez, with your support, honest critiquing, and pedagogical style, I grew more informed, more confident, and less sensitive than I

was before matriculating into the program. I cannot imagine going through this process or program without you. I am and will be forever grateful to have crossed paths with you. Thank you. Thank you. Thank you. 
To my boss, Maria Santacruz: Thank you for your unwavering support throughout my academic career. Your understanding and flexibility were extremely pivotal. I am thankful to have had you as my supervisor over the years.

To my Soror, Dr. Juanita Morrow: Thank you for your support, energy and constructive criticism. I also want to thank you for being a wonderful role model. Oo-oop!

To my friend since middle school, Dr. Michelle Martin-Romero: Thank you for gifting me your time and support, from across the country, during some of the busiest times of your professional life. Thank you for finding the time to squeeze me in when I needed your feedback.

To Dr. Fabian Tata: You gave me my very first job in higher education at Florida State University when I was just a freshman- I appreciate you for introducing me to the world of higher education and for always speaking life into me.

To Judith Thompson, James E. Wilkening, and again Dr. Fabian Tata: The three of you graciously wrote letters of recommendation in support of my journey to obtain this terminal degree. I thank you for believing in me and for putting your name on the line to get me to this point in my life. Thank you very much.

To all of my friends: Thank you for your understanding; thank you for not giving up on me in the many moments I passed up on opportunities to hang out and bond because I had class, an assignment, research, or was just overall mentally drained from school. Thank you for continuing to have my back and continuing to be there for me despite my absence. 
To each and every one of my participants: Thank you for entrusting me with your experiences. Thank you for your time, honesty, and transparency; I appreciate your willingness to meet with me. I would have been unable to conduct this research without you all. Thank you.

Finally, I am indebted to Florida International University: Thank you for funding this degree by way of the FIU Employee Tuition Waiver. The tuition waiver made this all possible and is one of the greatest benefits I have encountered throughout my professional career. Thank you. Go Panthers! 


\begin{abstract}
OF THE DISSERTATION
THE EXPERIENCES OF UNDERREPRESENTED FACULTY AND

ADMINISTRATORS: A STUDY OF CULTURAL TAXATION
\end{abstract}

by

Katrina Ngozi Amie

Florida International University, 2020

Miami, Florida

Professor Benjamin Baez, Major Professor

Cultural taxation is a term used by some to describe the additional work required of faculty of color as representatives of their racial or ethnic groups. Padilla (1994) defines cultural taxation as "situations that are imposed [on faculty]... by the administration.” Many prior studies about cultural taxation focus on the experiences of faculty of color at predominately White institutions and understanding of this phenomenon is heavily aligned with those spaces and circumstances. While much of the literature presents the additional workload for underrepresented faculty as damaging (Hirshfield \& Joseph, 2011; Pololi et al., 2010; Hassouneh et al., 2014; Mahoney et al., 2008; Kolade, 2016; Trower, 2003; Blackburn \& Lawrence, 1995), some research suggests that this work exemplifies a form of critical agency or is otherwise fulfilling (Baez, 2000; Blake, 2018; Chang et al., 2016). This dissertation offers insight into such understandings, but it expands the notion of cultural taxation by accounting for the experiences of administrators. Administrators were largely absent from literature about this phenomenon, although they, too, play significant roles within institutions of higher education. Thus, in the study, cultural taxation is defined as the additional physical or 
mental demands imposed on underrepresented members of the academy irrespective of intentionality or origination.

The setting for the dissertation research was Florida International University, which offered a sample that allowed for the inclusion of otherwise often excluded populations. Fifteen underrepresented faculty and administrators participated in this qualitative interview study by sharing their experiences at FIU as they relate to cultural taxation in the course of one semi-structured, open-ended interview session.

This dissertation found three forces that can induce cultural taxation: external, internal, and social. The results from the study require a recognition of cultural taxation as more than a phenomenon that underrepresented members of the academy experience a result of situations imposed by the institution. In addition, understanding the phenomenon beyond the physical demands by also acknowledging the mental demands experienced by underrepresented populations also provides a space for dialogue about some of the less visible components of cultural taxation and further provides a foundation for systematic changes in the academy. 


\section{TABLE OF CONTENTS}

Subjectivity Statement.................................................

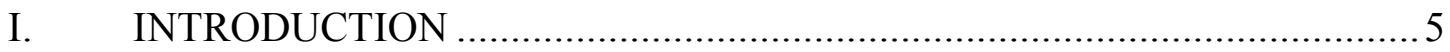

Cultural Taxation, Continuation of its Understanding ............................... 6

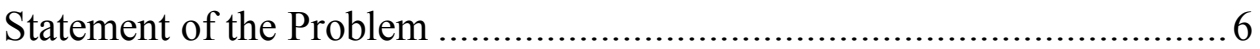

Purpose of the Study ………………….......................................... 9

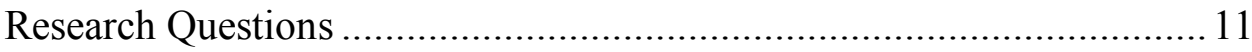

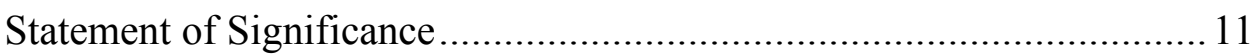

Limitations, Delimitations, and Assumptions of the Study....................... 12

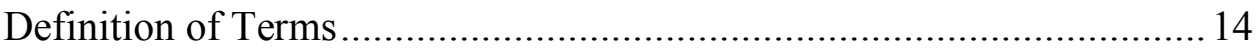

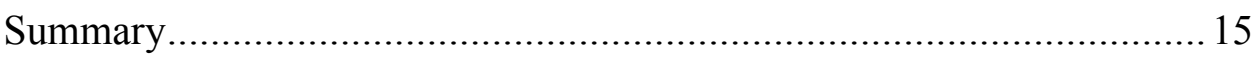

Organization of Study ..................................................................... 16

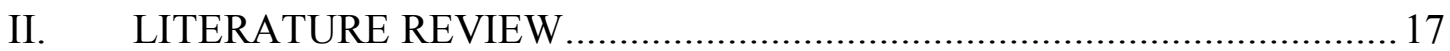

History of the Diversification of Higher Education................................. 19

Background on the term "Cultural Taxation." .........................................20

Components of Cultural Taxation ..............................................22

Consequences of Cultural Taxation............................................... 30

Cultural Taxation and Critical Agency .......................................... 32

Brief Overview of Hispanic-Serving Institutions ....................................... 33

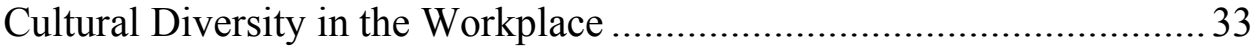

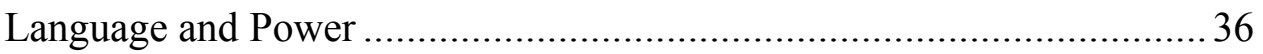

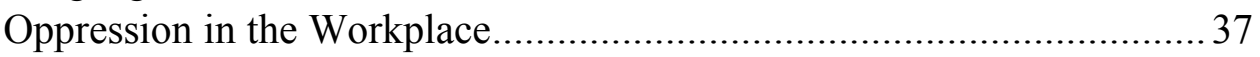

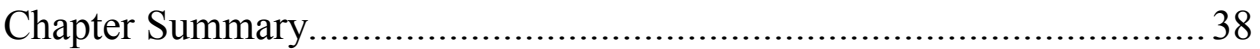

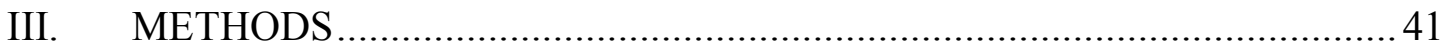

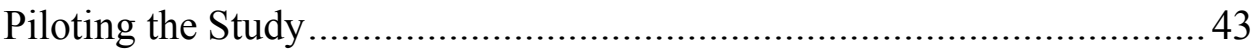

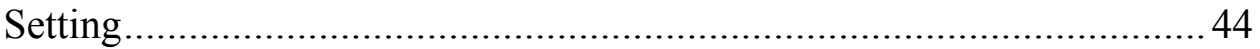

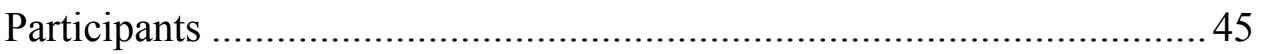

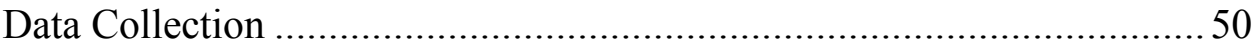

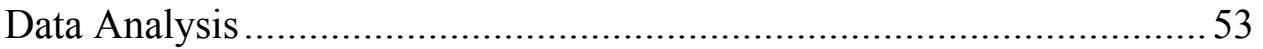

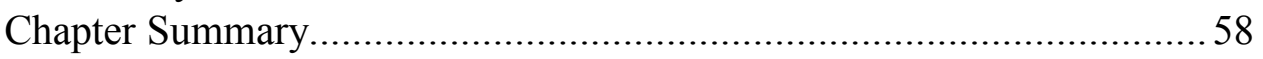

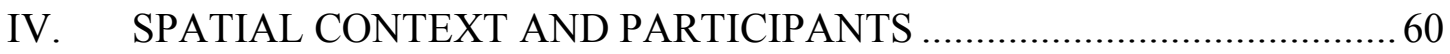

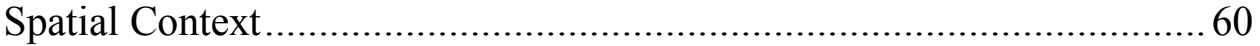

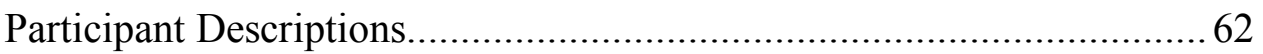

FIU as a Microcosm of the United States (Parallels) .............................. 84

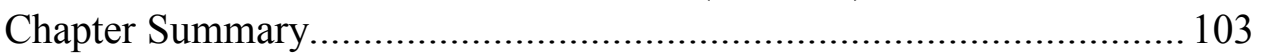

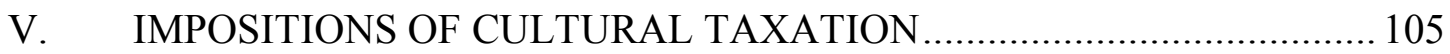

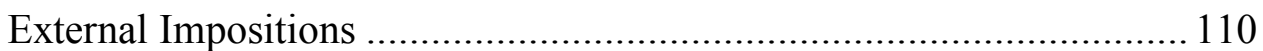

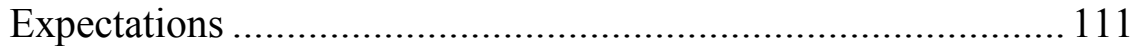

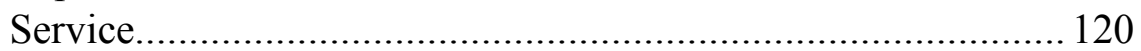




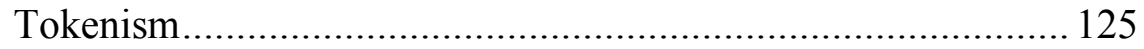

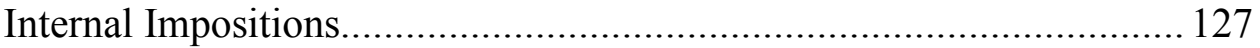

Obligation.................................................................. 127

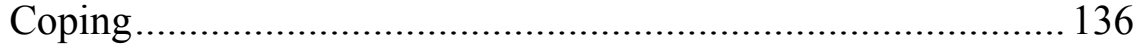

Critical Agency............................................................ 149

Social Impositions .................................................................. 153

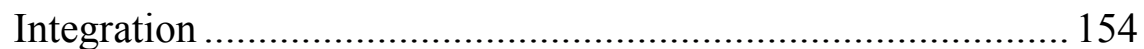

Additional Potential Impact of Cultural Taxation............................... 164

Chapter Summary........................................................................ 171

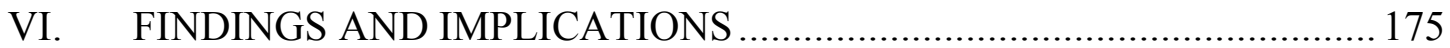

Summary of the Study ........................................................ 175

Extent Faculty and Administrators Experience Cultural Taxation ......... 178

Underrepresented Minority Faculty, Administrator and Cultural

Taxation at FIU............................................................. 179

The Economics of the Cultural Tax .................................................. 182

The Allocation of the Cultural Tax: For the Culture and For the

Institution .................................................................... 183

Cultural Taxation as Workplace Oppression ............................ 185

Categories/Types of Cultural Tax ..................................................... 186

Implications and Recommendations for Practice............................... 189

Recommendations for Future Research ........................................... 192

Final Thoughts and Concluding Remarks ....................................... 195

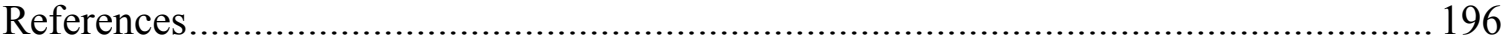

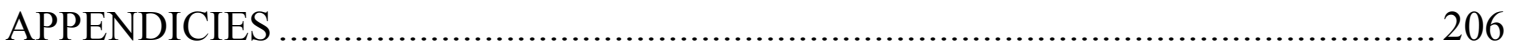

VITA 


\section{Subjectivity Statement}

I want to open the study by sharing a bit of my personal story. I believe that transparency is important because of the nature of the topics addressed in my study, and the fact that I share similar experiences as some of the participants I had the pleasure of interviewing. I am a first-generation, Nigerian-American woman. These intersectional classifications have influenced my journey as both a student and a working professional. As a student, particularly first-generation and Black, I have found myself craving for guidance, representation, and/or merely a connection to anyone I could relate to ethnically or culturally. Thus far, I have attended Florida State University (PWI), the University of Central Florida (PWI) and currently, Florida International University (HSI). All three institutions have one notable commonality to me: their tendency to confirm and magnify my minority status. I felt this magnification in classrooms and general campus life. As a professional who has also worked in spaces where I also felt this magnification, I can say that I have found myself experiencing the same demands and expectations I did while pursuing my Bachelor of Science in Health Services Administration and my Master of Art in Educational Leadership.

One of the most important aspects about myself that brought me to this current moment in my life is my thought process. I am always in a constant state of selfevaluation; I jokingly refer to myself as a walking autoethnography. For this reason, I have come to have a solid understanding of myself and my experiences in life. These experiences and my understanding of them have the potential to limit me in my study, but I used my awareness of the potential limitation to my advantage and focused my energy 
towards avoiding allowing my experiences to impede my understanding of my participants' experiences.

I recognize that as I evolve into a more advanced professional, these "cultural taxations" are something that I owe to my field, the students progressing behind me. I am aware of the burdens - and I use that term loosely - this commitment entails, but I am a firm believer in the statement "To whom much is given, much is expected." Despite any socially-induced struggles I have encountered during my academic and professional career, I attribute my growth and cultural consciousness to them. I intend to use them as tools in my new and ever-evolving journey as an agent for social transformation, which I refer to as a moral obligation.

I chose to investigate "cultural taxation," specifically, instead of the overall experience of the participants, because the phenomenon is traditionally used to refer to minorities in predominately White settings, which allowed for the expansion of the phenomenon's understanding and application. I wanted to explore whether and how the phenomenon is experienced in an interminority setting. Florida International University is a Hispanic-Serving Institution with a majority Hispanic/LatinX workforce. I wanted to investigate how this form of taxation might be experienced among minorities when there is a dominant minority group.

Throughout the study, I found many of the interviews to be therapeutic for me; they reduced a degree of isolation I felt at times. I felt less alone within the institution, as I discovered that others were experiencing similar situations similar to mine. The interview process did, to a certain degree, remove my mental blinders. My reference to "mental blinders" is not to say that I was oblivious to certain situations, but certain 
situations and/or experiences did not weigh as heavily on my mind as they might have following the interviews. For instance: When I started my job at FIU I was certain that I was hired because of my capabilities, my resume, and what I could bring to the department. I would like to preface that I am the only Black person in my department. After sitting with my participants, who in many cases are also the only person from their ethnic or racial background in their respective workspaces, and having them recall instances in which people have told them to their faces that "We hired you because we needed diversity," or "We hired you because of your race" really kind of put some things into perspective for me and my experiences. During the study, I found myself feeling a combination of empowerment, depression, anger, annoyance, drain, and confusion following the sessions with my participants.

Coming from a household in which I was always praised and taught that I am just as good as anyone else, and of really being a cognizant of my abilities growing up from childhood to adulthood, it was almost impossible for me even to consider that someone would hire me simply because of my race. But, then again, I do come from a household in which one of my parents come from a homogenous country. There were stark differences between my participants that were from homogenous countries and those from the United States as it related to their understanding of their experiences with cultural taxation. So, I think my cultural connection to a homogenous country, too, may have influenced my prior understanding of myself and certain experiences.

I would also like to start by recognizing that Florida International University has been recognized by The Chronical of Higher Education as one of the "Great Colleges to Work For" and Forbes "Best Employers for Diversity." With that, the experiences 
presented in the study do not serve to discredit any of the institutions' efforts or accolades. I have been an employee at the institution for just over four years, and I am very grateful for the knowledge, experience, and skills that I have gained during my time at the institution. 


\section{CHAPTER I}

\section{INTRODUCTION}

The demographic makeup of full-time faculty at four-year colleges highlight disproportionate over- and under-representation with regard to race and ethnicity (Meyers, 2016). According to the National Center for Education Statistics, of the male and female faculty, $76 \%$ of all faculty are White, while $10 \%$ are Asian/Pacific Islander, $6 \%$ are Black, $5 \%$ are Hispanic, $2 \%$ are American Indian/Alaskan Native, and $1 \%$ are of mixed races or ethnicities (2016). Scholars have argued that "probably few policy areas of higher education have received more recent attention than the issue of race on campus" (Hurtado et al., 1998, p. 279). Although there have been efforts to diversify the faculty across the country, faculty of color are nevertheless still underrepresented in academic work spaces, and their accomplishments often go unnoticed (Turner \& Gonzalez, 2008). The disproportion in racial makeups has the potential to create an environment that could lead to cultural taxation.

Amado Padilla coined the term cultural taxation and defines it as an "obligation to show good citizenship towards the institution by serving its needs for ethnic representation on committees, or to demonstrate knowledge and commitment to a cultural group" (Padilla, 1994, p. 26). These obligations occur because of one's racial or ethnic differences. Cultural taxation is a phenomenon experienced by minorities predominately, but not exclusively. As long as one has a commitment to diversity-related matters, White faculty, too, can fall "victim" to cultural taxation. "Victim" is in quotation marks because while much of the literature presents the additional workload on underrepresented 
minority faculty as damaging, there is literature that presents some acts related to the phenomenon as forms of critical agency or otherwise fulfilling (Baez, 2000; Blake, 2018; Chang et al., 2016).

\section{Cultural Taxation, Continuation of its Understanding}

Following my accounts with the participants, I found that it was necessary to adjust how I was defining cultural taxation to encompass the totality of their experiences. The fundamental definition, as presented by Amado Padilla (1994), defines cultural taxation as "the obligation to show good citizenship towards the institution by serving its needs for ethnic representation... or to demonstrate knowledge and commitment to a cultural group." The initial/ evolved definition I had been utilizing for the study was "Anything done or expected of one outside of their job duties because of their cultural, racial, or ethnic differences." The final definition I developed and come to use to define the phenomenon and its multidimensionality is "The additional physical and/ or mental demands imposed on underrepresented members of the academy irrespective of intentionality or origination."

\section{Statement of the Problem}

Some members of academe intentionally participate in activities, which Padilla would identify as forms of cultural taxation, as a way of resisting hegemony (Baez, 2000). These kinds of calculated actions can be valuable when working to disrupt unfavorable situations. Recognizing the intentionality of these acts against hegemony lends itself to a greater understanding of Minority-Serving Institutions (MSIs). MinorityServing Institutions are rooted in a desire to serve minority students with a particular interest on a "completion agenda" (Harmon 2012). To aid in the completion agenda, 
many MSIs commit to hiring minority faculty and staff, creating opportunities to foster mentorships with students, which has a connection to student success (Harmon, 2012). Minority-Serving Institutions often afford the best fit for underrepresented students, which in turn improves their chances of graduating.

Research on cultural taxation has been focused on predominately White institutions. As a result, the understanding of this phenomenon is heavily aligned with those environments. The need to study cultural taxation across institutional-type, including MSIs, is imperative-This need is independent of other institutional classifications (i.e., Doctoral Universities, Master's Colleges and Universities, Baccalaureate Colleges, Associates Colleges, and Special Focus Institutions), as these classifications present their own additional set of characteristics that could influence how cultural taxation takes place. The need to study cultural taxation across institution-type is also in relation to the racial and ethnic demographics of institutions. Minority-Serving Institutions serve a significant number of low-income students (Li, 2007), which compels faculty to offer help and become role models. Moreover, underrepresented minority students are more likely to be less prepared for college due to the likelihood of having had attended poorly funded K-12 schools (Harmon, 2012), similarly requiring more work on the part of the faculty helping these students. These characteristics have the potential to create additional challenges for both faculty and administrators at MSIs.

The ways in which underrepresented members of the academy perceive and handle cultural taxation is essential because of their underrepresentation. Underrepresentation can be the case in non-predominately White settings; it takes place 
whenever there is the presence of a dominant cultural, racial, or ethnic group, and so it can exist in MSIs, depending on the institutions' demographic makeup.

As it stands, studies have extended the components of cultural taxation to include experiencing irregular expectations, obligations for mentoring, coping with colleagues' problematic behaviors, legitimating ones' academic credentials, and acculturating, all of which could lead to over-commitment, feelings of isolation, and impediments in career progress (Hirshfield \& Joseph, 2011; Pololi et al., 2010; Hassouneh, Lutz, Beckett, Junkins, \& Horton, 2014; Mahoney, et al., 2008; Kolade, 2016; Trower, 2003; Blackburn \& Lawrence, 1995). The aforementioned components entail extensions of "cultural taxation's" original application. Most studies provide instances of cultural taxation at predominately White institutions.

There are three notable gaps in the literature regarding cultural taxation. The first is the lack of studies on administrators. Administrators, however, are critical constituents in higher education, and those from ethnic and racial minority groups could be subjected to cultural taxation. The second gap is a lack of studies on faculty (and/or administrators) who may not classify as a "minority" under state or federal classifications but who also experience cultural taxation as an underrepresented minority in their respective environments. White faculty and administrators in higher education can be underrepresented in certain spaces and may face cultural taxation as well. The third gap in the literature is the lack of studies on minority-serving institutions. I did not come across research on the existence of inter-minority relations or how cultural taxation affects underrepresented minorities in minority-serving institutions. 
Understanding the effects of cultural taxation for both faculty and administrators, regardless of racial and ethnic background in minority-serving institutions, therefore, is essential. With the current faculty demographics, there is a clear need for instituting additional diversity at all levels within institutions and for understanding what diversity may entail for underrepresented incumbents.

The gaps in the literature accompanied by the complexity of cultural taxation warranted the use of qualitative inquiry. Qualitative inquiry creates an opportunity to gather "thick description" (Geertz, 1973) about a phenomenon which allowed me to grasp multiple understandings of cultural taxation and how underrepresented faculty and administrators experience it. In addition, qualitative inquiry also allowed me to capture unique feedback from my participants about the phenomenon which was important especially given the unique demographic of the institution (FIU) — unique in that the demographic of the setting differs from the demographic of institutions explored in prior literature. Furthermore, the qualitative approach amplified the voices for individuals whose experiences often go unheard.

\section{Purpose of the Study}

The purpose of the study was to extend the application of the notion of cultural taxation outside of its traditional use with regard of faculty of color in predominately White settings. For the study, because of the evolution and broad application of the term, cultural taxation is being acknowledged as anything done, or expected of one outside of their job duties because of their cultural, racial, or ethnic differences. Cultural taxation is typically bestowed upon ethnic minorities; however, not in exclusivity. An ethnic minority as defined by the Cambridge dictionary is "a group of people of a particular race 
or nationality living in a country or area where most people are from a different race or nationality," or "a subordinate group whose members have significantly less control or power over their own lives than do the members of a dominant or majority group" (Schaefer, 2012, p.5). These two, geographic and sociological, definitions will serve as the point of reference for the study.

My dissertation sought to explore a more extensive application of cultural taxation to include faculty and university administrators at Florida International University (FIU), a MSI with a workforce demographic composition that predominately consists of members that would classify as minorities. The institutions faculty and staff demographic makeup for minorities as classified by the US Census (Asian, Black, Hispanic/LatinX, Not reported, Pacific Islander, and Two or more races) accounts for $77 \%$ of the settings' total count.

The qualitative study utilized interviews to probe into the experiences and perceptions of cultural taxation among underrepresented faculty and administrators at Florida International University, Modesto Maidique campus. Studying cultural taxation at Florida International University allowed me to explore the phenomenon amongst a demographic that is largly absebt from literature. The use of interviews was important because it allowed me to gather thick description, capture unique feedback, and share the voices of my participants. Additionally, the use of interviews allowed me to control the direction of the study. I was able to use my interview protocol as an outline for the topics I wanted to cover, and using the participants responses, I was able to further direct the interview to obtain additional detail about their experiences which allowed me to identify new ways of understanding the phenomenon. 


\section{Research Questions}

1. To what extent do faculty and administrators experience cultural taxation at Florida International University, Modesto Maidique campus?

2. How do the experiences of faculty and administrators compare with regard to cultural taxation?

\section{Statement of Significance}

The findings of the study contribute to a more extensive understanding of cultural taxation outside of its traditional uses by exploring the experiences of faculty and administrators at a Hispanic-Serving Institution (HSI). The study also provides insight on where cultural taxation, or the pressure to participate in certain activities, develop prior to becoming part of one's experience. The continued diversification of higher education creates a greater demand from underrepresented faculty and administrators (often neglected in studies about cultural taxation). Prior studies have explained the impact cultural taxation has on faculty of color as it relates to career progress, job satisfaction, and burnout. Understanding the potential impact on administrators as well can help members of academia understand the experiences of these individuals who, too, play important roles within the university.

The primary targeted audience for this dissertation are members at all levels in higher education who encompass authority figures, decision makers, students, administrators, faculty members, and senior leadership councils. My study hopefully contributes to a degree of their understanding of what underrepresented members of academia may experience in their daily work lives. The information can be beneficial when it comes to potentially uninformed demands and/or expectations placed on 
underrepresented members of an institution. The expanded application of the term could also promotes policy recommendations that can enhance the experiences of underrepresented faculty and administrators in higher education across institutional type as it relates to tenure, retention, career growth, and mental health which would entail current policies undergoing review to explore how they might perpetuate inequities among underrepresented faculty and administrators.

The secondary audience, because of the more flexible application of the term cultural taxation, and the data provided by the participants in the study, would be anyone that identifies as a member of a dominant group in their respective spaces to aid in understanding how their actions and/or behaviors, regardless of intentionality, influence the experiences of their underrepresented counterparts. The tertiary audience is for professionals in various fields who work with underrepresented minorities, as the findings in the study aspire to have a degree of transferability across industries.

\section{Limitations, Delimitations, and Assumptions of the Study}

Limitations are "potential weaknesses or problems with the study identified by the researcher" (Creswell, 2005, p. 198), while delimitations limit the scope and define the boundaries of a study (Simon, 2011). Assumptions, on the other hand, are somewhat out of the researchers' control but could impact the relevance of a study if they were not present (Simon, 2011).

Limitations

A limitation of the study related to the method being utilized; some of the participants appeared to lack a degree of comfortability in expressing their experiences during interviews. This could be attributed to the nature of the inquiry, the fact that they 
are, and in some cases were, employed by the institution, and a lack of trust, potentially. I make an assumption about the lack of trust because one theme that arose among a number of participants was, despite any negative experiences they expressed, the tendency to include positive comments about their boss; these disclaimers were made across races, genders, and ethnicities. Cross-racial interactions did not seem to create a significant challenge as it related to comfortability and openness. In an effort to minimize this challenge, what I understood to be a potential limitation, I often relied on the fact I am also an underrepresented minority to establish some degree of trust and openness during the interview processes. I also made sure I managed my responses, reactions, and facial ques to avoid making the participants feel judged. One thing I did notice during my cross-racial interactions was a tendency among the non-Black participants to inform me that they understood racial dynamics before answering some interview questions. I cannot argue that it did or did not impact the truthfulness of their responses, but some of the participants did seem to find it necessary to make me aware of their racial awareness. Delimitations

The study focused on underrepresented faculty and university administrators at Florida International University (according to the institution's demographic makeup), and more specifically the Modesto Maidique Campus. Additionally, the sample only consisted of current faculty and university administrators within the institution. This gave me the perspective of current employees but excluded that of those who may have experienced cultural taxation who are no longer with the institution. 
Assumptions

A qualitative interview study assumes that the participant selection criteria are appropriate; hence the participants selected for the study did perceive and identify as underrepresented within their respective spaces, have experienced the phenomenon, and were able to speak on it. It is also assumed that participants were be able to express meaning and understanding through interviews. Also I assumed that the participants were open and honest about their experiences, which are ultimately shaped by how the participants make meaning of them.

\section{Definition of Terms}

Cultural taxation: Anything done or expected of one outside of their job duties because of their cultural, racial, or ethnic differences. It will also be used in this dissertation to represent the additional physical and/ or mental demands imposed on underrepresented members of the academy irrespective of intentionality or origination.

Interminority: The coexistence of different minority groups as classified by the United States Census Bureau. It will also refer to situations in which minorities work among minorities.

Interminority Setting: The institution (HSI) (FIU MMC) will serve as the interminority setting as the demographics of the institutions represent a majority-minority makeup composed of different minority groups

Underrepresented Faculty and Administrators: Any member that is not classified as a member of the dominant group within the institution. Because of the unique demographic makeup of FIU, comprising of minorities who are actually in the majority, the term will be utilized to represent the participants in the study. 
University Administrator: A member of the university administration. This classification refers to an individual employed by the university who is responsible for running, maintaining, or supervising the institution and includes multiple levels of staff.

Underrepresented Minority (URM): A member of any racial or ethnic group that is a part of "a subordinate group whose members have significantly less control or power over their own lives than do the members of a dominant or majority group" (Schaefer, 2012, p.5) outside of the traditional parameters that typically account for AfricanAmerican/Black, Asian, Hispanic/LatinX, Native American/Alaskan Native, and Native Hawaiian/Other Pacific Islander.

\section{Summary}

The disproportion in racial and ethnic makeups in higher education can lead to the cultural taxation of underrepresented members in academe. Previous studies have presented components, consequences, and benefits of the phenomenon among faculty of color at predominately White institutions. The study is important because it created an opportunity to investigate the phenomenon at a non-PWI among an alternative demographic which allows for the inclusion of otherwise excluded participants. Additionally, the study provided a space to give administrators a voice about their experiences regarding cultural taxation, which is important because of the significance of their roles in higher education. In addition, the way that I apply the term cultural taxation allows for the exploration of otherwise overlooked elements of the phenomenon that have not been recognized in prior literature. Having a multidimensional understanding of cultural taxation is important because as institutions work to mediate inequities in academe, knowing how it plays out in alternate ways and environments could aid in 
insuring equity across institutional-type. Furthermore, knowing how cultural taxation might be experienced across position-types (faculty and administrators) provides an opportunity for equity at multiple levels within higher education. The expansion of the application of the notion of cultural taxation outside of its traditional norms allows for new understandings and practices in the academy.

\section{Organization of Study}

Chapter 2 will present a review of literature significant to the study. It will focus on available literature about the experiences of minority faculty in higher education as they relate to cultural taxation, as well as literature selected to aid in understanding some of the data provided by the participants. Chapter 3 will discuss the methodology of this qualitative interview study, as well as its research design. Chapters 4 and 5 will explore the results of the study, which include the extent to which the experiences of faculty and administrators within the institution relate to cultural taxation and the multiple ways it can be experienced and the socially-induced forms of the phenomenon that were largely absent from the prior literature. Finally, chapter 6 will present a comparative view of the experiences of underrepresented faculty and administrators in the study, significant findings, implications, and recommendations for practice and future research. 


\section{CHAPTER II}

\section{LITERATURE REVIEW}

This review of literature will open with a brief history of the evolution of diversity in higher education. Following this, the text will provide background on the phenomenon of cultural taxation and then explore relevant literature related to, or as a result of, the phenomenon organized by common topics to include: Irregular expectations, mentoring, coping with colleagues' problematic behaviors, differential legitimacy, and acculturation. Tokenism is also presented in this review of literature as it was explicitly articulated by some of the participants as a part of their experience, and I argue, later in the text, that it can be understood as a form of cultural taxation. Afterward, the chapter will address common results of the phenomenon to include over-commitment, feelings of isolation, and impediments in career progress. The review of literature will continue with the explanation of cultural taxation and how it can relate to critical agency, an outlook that differs from much of the published literature. The review of literature then presents a brief overview of the history of Hispanic-Serving Institutions and Florida International University. Throughout many of the interviews, the topic of language arose, as a result, chapter II will also include a section on language and power to ground the participants' experiences. The chapter concludes with a section addressing workplace oppression, which is included to set a foundation for an understanding of how cultural taxation can also be understood in relation to oppression in the workplace.

Because all of the themes or forms of cultural taxation presented in the literature are not recognized in the original context of the phenomenon, I provide a rationale as to why I have chosen to include them at the end of each subsection. The articles I selected that do 
not directly identify cultural taxation as their focus were selected because they correlate with the original "identifiable forms," or the overall essence of cultural taxation as cultural taxation can be understood as an umbrella term for a series of actions and behaviors. The forms of cultural taxation conveyed in this review of literature are not being argued as stringent entities that define the phenomenon. It is essential to recognize that cultural taxation can take many forms. One aspect of these "components" that unify them is their ties to pressure and time; Pressure from within, pressure from others, and pressures induced by the environment, which require time to oblige them.

\section{Approach to the literature}

In searching for literature, I utilized education, medical, and sociology databases to include: JSTOR, ERIC, SAGE, NCBI, AJS, and TandF. There was a limited amount of scholarly literature that addressed cultural taxation so I used following keywords to encapsulate the phenomenon of cultural taxation as many researchers have not utilized the term itself, but have explored the essence of the phenomena be it in fragments or holistically: cultural taxation, minority tax, minorities in higher education, underrepresented minorities, cultural obligation, invisible labor, race-related service, faculty exploitation, critical agency in higher education, minority faculty experience, racial fatigue, and Black tax. To support the findings in the study, I used the following keywords: diversity, workplace diversity, oppression, workplace oppression, language and power, minority-serving institutions, Hispanic-serving institutions, tokenism, minority on minority discrimination, and critical agency. From preliminary searches, I was able to link myself to other sources listed in the reference lists in the literature that appeared to be valuable to my research needs. 
Also, because of the nature of the literature and scope of research on cultural taxation, the terms faculty "of color," "minority," and "underrepresented" minority (URM) will appear interchangeably throughout the text, as presented in different research findings. Although these terms differ, their essence is essentially the same in this particular area of research.

\section{History of the Diversification of Higher Education}

According to Brubachar and Rudy (1997) and Lucas (2006), the diversity of students in higher education has increased in the United States since its beginnings at Harvard College in 1636 when access to education was limited to White males. As time progressed, institutions of higher education have increased their continuum of diversity by way of class, gender, and race. The equity of opportunity is a component of the democratization of higher education. Historically, one of the landmark beginnings of the diversification period took place between 1850 and 1890; this can be attributed to the Morrill Land Grant of 1862, which created land grant colleges and public education, and the Morrill Act of 1890.

In 1825 , the University of Virginia became the first public university in the United States; this contributed to breaking down class barriers in higher education. The Morrill Act of 1890 (also known as the second Land Grant Act) increased federal aid to land grant institutions. It penalized those that discriminated against African-Americans with regards to admissions, thereby breaking down racial barriers in higher education.

The diversification of higher education can also be attributed to the emergence of institutions explicitly created for African-Americans, i.e., Historically Black Colleges and Universities (HBCU), women's colleges, and tribal colleges. Initially, on the basis of the 
outcome of the Plessy v Ferguson case in 1896, racial segregation was constitutional given facilities were "separate but equal." The Separate but Equal doctrine was later undone as a result of the Brown v. Board of Education case of 1954, which ruled such segregation unconstitutional.

With regard to race, the number of students of color diversifying institutions of higher education have bypassed the diversification of faculty of color in institutions that are not designed to serve their respective minority group (i.e., Historically Black colleges and universities, Hispanic-serving institutions, Tribal colleges or universities, Alaska Native or Native Hawaiian serving-serving institutions, predominately Black institutions, Asian American and Native American Pacific Islander-serving institutions, and Native American-serving nontribal institutions). Turner and Gonzalez (2008) explain that "campuses across the country are engaged in efforts to diversify the racial and ethnic makeup of their faculties," however [the] efforts are perhaps the least successful of campus diversity initiatives as faculty of color remain underrepresented and their achievements in the academy almost invisible" (p. 139).

\section{Background on the term "Cultural Taxation."}

The diversification of higher education, the underrepresentation of faculty of color (FOC), as well as the FOC to the student of color ratio, has created an environment in academe that places multiple responsibilities on FOC in addition to what is prescribed in their position description. These additional responsibilities and expectations are referred to as cultural taxation. As presented earlier in the dissertation, Amado Padilla originated the term to describe a phenomenon in higher education for "ethnic scholars." 
While cultural taxation is traditionally a term applied to ethnic minorities, it can apply to any members of the academy who have a moral commitment to diversity.

Amado Padilla addresses the impositions placed upon "ethnic scholars" as a result of their race, ethnicity, or presumed knowledge of cultural differences. Padilla outlines six "identifiable forms" of cultural taxation to include: being called upon to serve as the "expert" on diversity matters, being called upon to educate members of a majority group about diversity, serving on affirmative action committees without any observable changes, serving as the liaison between the institution and the ethnic community even though one may not agree with the institutions' policies, taking responsibility for settling problems regarding sociocultural differences among the members of the institution, or being called upon to serve as a translator or interpreter when the institution is presented with non-English speakers (Padilla, 1994, p. 26).

Upon identifying the six forms of cultural taxation, Padilla refers to one's responsiveness to them as a deep sense of "cultural obligation." The concern expressed in literature surrounds the effect these cultural obligations, or burdens, have on faculty. These structural inequalities often go unspoken and have been noted to impact the tenure and promotion of faculty members of color. These forms of cultural taxation can be a means of survival (Canton, Cintrón, \& Jacobs, 2002), can take place as a result of one's dedication to, or interests in, diversity issues, or happen directly and inevitably as a result of one's racial, ethnic, or cultural identity. Cultural taxation is described as a form of “invisible work” (Lechuga, 2012).

It is important to note that this phenomenon is experienced predominately by minorities. Additionally, since the acknowledgement of the phenomenon (cultural 
taxation), researchers have expanded the forms of cultural taxation to include: Irregular expectations, mentoring, coping with colleagues' problematic behavior, differential legitimacy, and acculturation. These circumstances have been known to result in overcommitment, feelings of isolation, and impediments in career progress. These particular aspects, coupled with Amado Padilla's original understanding, guided the study.

The components are reflective of studies involving underrepresented faculty in predominately White settings. The studies are not representative of diverse institution types. Cultural taxation is a phenomenon experienced by minorities predominately, but not exclusively. As long as one commits oneself to diversity-related matters, they too can fall "victim" to cultural taxation. The study to explored cultural taxation in a nontraditional environment, i.e., at FIU, MMC.

\section{Components of Cultural Taxation}

Faculty of color can face unequal and irregular expectations with regards to research preferences, participation in diversity committees, teaching race-related courses, being an expert on diversity matters, and serving as advocates for students of color. The expectations can lead to over-commitment from the "burden" of taking on too many responsibilities. Traditional forms of cultural taxation are often perceived as being a disadvantage to faculty of color because they are held to the same research standards as their non-minority counterparts but are also expected to complete additional tasks with regards to their respective minority groups (Hirshfield \& Joseph, 2011).

Preconceived racial expectations (typically lower) contribute to minority faculty warranting the need to prove their "value, worth and ability" (Campbell et al., 2015, p. 2), unlike their White counterparts. URM faculty feel the need to work harder to gain respect 
and "be perceived as accomplished" (Pololi et al., 2010, p. 1635). Minority faculty also express being stereotyped (Pololi et al., 2010, p. 1365). Snyder (1982) discusses the impact of stereotypes and how they impact lives and social interactions in the classroom and workplace. He also expels how they become normalized and refers to the "selffulfilling nature of stereotypes" (p. 543). Faculty of color are not typically afforded the privilege of assumed individuality. Their individualism is secondary to their respective racial or ethnic group membership (Hassouneh, Lutz, Beckett, Junkins, \& Horton, 2014, p. 4). Additionally, minority faculty express that their colleagues and supervisors house reduced expectations concerning their capabilities.

Expectations also exist with regard to participating in diversity-related efforts and/or service. Minority faculty members express being subjected to institutional pressure to be active participants in matters related to diversity, although there are recognized "gap[s] between intention and implementation" (Mahoney, Wilson, Odom, Flowers \& Adler, 2008, 784). These expectations have been noted to be unrealistic.

Expectations also extend into research interests. Minority faulty report stereotypical expectations to participate in race-related research (Hirshfield \& Joseph, 2011). Along with the expectation to produce minority-related research are the perceptions that accompany it. These perceptions differ between minority and nonminority faculty (Johnson-Bailey \& Cervero, 2002). Specifically, "Black faculty members are often seen as having an agenda when they pursue this line of research, whereas White scholars are seen as progressive" (Johnson-Bailey \& Cervero, 2002, p. 23). Institutions can also have hidden service agendas (Brayboy \& McKinley, 2003); some service agendas revolve around tokenism. 
Tokenism has been described as "A mechanism by which inequalities are unjustly maintained" (Grant, 2017, p. 2). The Merriam Webster Dictionary defines tokenism as "the policy or practice of only making a symbolic effort." It can give the illusion of diversity despite disproportionate representation. Smith (1989) explains that "As long as some groups are underrepresented, the experience of tokenism- including isolation and heightened visibility- will emerge." The heightened visibility can lead to individuals being "marked." McKinley (2003) argues that bodies of color, or as it relates to the study, underrepresented bodies are "marked bodies whose presence implements diversity" while their "White (and in the case of the study, White and/or predominate) faculty and administrators have already done their part by hiring faculty of color; their bodies remain unmarked in the process" (p. 74). Being "marked" means that these bodies of color carry or are expected to carry additional responsibilities related to, or in promotion of, diversity efforts, thereby subjecting themselves to cultural taxation. Tokenism, if understood as a symbolic attempt to serve the institutions' need for ethnic representation, could be understood as a component of cultural taxation.

I argue that expectations fall into a form of cultural taxation as it relates to the energy required to either meet or combat them. The time and effort it takes to battle with presupposed expectations alongside effectively completing one's job duties can serve as an additional responsibility. There is an interesting dichotomy of expecting more from faculty of color while subconsciously expecting less of them.

Serving as a mentor or being mentored by another professional appears to be a common theme and projector of one's career path. Two subsets of mentoring present themselves in the literature: Faculty-Student mentorship and Faculty-Faculty mentorship. 
Mentorship is one of the ways minority faculty provide support to minority students, and often echoes a feeling of obligation toward one's community (Mahoney et al., 2008, p. 781). Sometimes underrepresented minority (URM) faculty are taxed to serve as mentors to other URM students and staff without having adequate mentorship themselves. The relationship between minority faculty and minority students "help to dispel myths and stereotypes about people of color," and as a result, enhances their experiences as minorities (Hassouneh et al., 2014, p. 2).

Adequate mentorship for minority faculty as a disparity surfaced throughout the literature. Having a mentor can impact one's success; it enhances the feeling of belonging and support as the process of navigation and promotion are traditionally complex in higher education. "[Mentorship] is especially significant as many African-American and Hispanic faculty come from backgrounds where they had little exposure to academia or the systems of higher education" (Pololi et al., 2010, p. 1365). The literature relays that "The absence of mentors may contribute to URMM underrepresentation in academic medicine" (Rodríguez, Campbell, \& Pololi, 2015, p. 2), which can be applied across academic areas. Minority faculty indicate that mentoring is key to one's professional success, and also link it to career satisfaction. It provides a sense of integration as well as cultural and emotional support. The demand and criticality of having minority mentorship are often met with shortages (Mahoney et al., 2008, 784). Although faculty of color expressed their lack of role models, they still feel obligated to serve in leadership roles and provide mentorship for other URM faculty (Pololi et al., 2010).

Mentoring can be a form of cultural taxation because of the disproportionate amount of minority faculty to minority faculty and/or minority faculty to minority 
students. What this has the potential to do is place a more significant burden on those few faculty members of color that may already lack their own form of mentorship to mentor those behind them, be they students or other faculty. However, in an effort "to show good citizenship," "[serve the] needs for ethnic representation" and/or "demonstrate knowledge and commitment to a cultural group" (Padilla, 1994, p. 26), they extend themselves to serve as mentors and role models to others.

Faculty of color can also experience colleagues' problematic behaviors. These behaviors include colleagues ignoring race issues in department relations, being used by colleagues as a marketing tools to obtain institutional recognition or funding for "diversity," being met with racist comments or implications that faculty of color do not belong and dealing with colleagues who deny racism or discrimination (Hirshfield \& Joseph, 2011). As a result, minority faculty have had to develop coping skills ranging from confrontation to avoidance (Mahoney et al., 2008, 784). Expectations, as cited prior, too, can fall under these problematic behaviors.

Coping can be a form of cultural taxation as a means to maintain one's sanity or as an alternative to quitting. Coping entails staying in an environment that can be toxic in order to "[serve an institution's] needs for ethnic representation" and to "demonstrate commitment to a cultural group" (Padilla, 1994, p. 26). Overcoming toxic environments is pivotal to the evolution of diversity. If members of these minority groups do not exude fortitude in their daily work encounters, they risk minimizing the already minimal level of diversity, thereby allowing the perpetuation of the cultural taxing of their minority counterparts. 
Differential Legitimacy refers to the added burden of legitimating ones' academic credentials, and is broken down into four subcategories: feeling a lack of belonging in ones' department, being questioned about one's qualifications, not having one's research valued by way of negative or passive responses about their work, and feeling the need to prove oneself by working harder than their White colleges to earn their peers' respect. Literature indicates that professors of color face instances in which other scholars hold a degree of skepticism about their aptitude (Johnson \& Sadao). These beliefs are created by the perceptions that affirmative action policies give faculty of color favor over their White counterparts within institutions (Hirshfield \& Joseph, 2011). Pololi et al. (2010) address the stigma of having had or being assumed to have had participated in affirmative action programs (p. 1366). Similar to expectations, I argue that differential legitimacy is a form of cultural taxation in relation to the energy exerted to prove oneself beyond what is expected or presupposed, unlike members of the majority group.

In an effort to blend in, gain acceptance or progress in their academic fields, minority faculty find themselves attempting to mesh socially, psychologically and/or culturally to that of the majority group (Kolade, 2016, p. 112-113). The traditional academic model and culture of academe creates a "need" to acculturate; it portrays one way of doing things, including how to " ffit' into the department and be a good colleague" (Trower, 2003, p.6). Minority faculty express feeling pressured to participate in respectability politics (Mahoney et al., 2008).

LaFromboise's model of bicultural competence is rooted in the concept of second-culture acquisition and is geared towards creating an understanding of the stress that accompanies acculturation. The model has been used to explore the experiences of 
minority faculty members (Kolade, 2016). Data indicate a shortage of minority-race faculty members, which in turn creates a domino effect for prospective minority nursing faculty; the chain of events can be applied across academic fields. Additionally, the shortage warrants the likelihood of minority groups experiencing pressure to acculturate. However, "Neither separation nor integration can eradicate the meaning of difference as long as the majority locates difference in a minority group that does not fit the world designed for the majority" (Minow, 1990, p. 25).

Acculturation can be a problematic form of cultural taxation as it negates diversity efforts. While particular minority-group members are physically present in the institutions, they are not contributing to cultural diversity. Adopting that of another's recuses the presence of one's own. These actions are counterproductive and inhibit the cultural diversity of the academy.

Acculturation, in some instances, can also be viewed as a management control mechanism (Hewege, 2011). Understanding acculturation as a means of control and control as a form of discipline lends itself to Foucault's (1977) analysis of discipline as a number of practices by which the body's actions can be controlled. Practices, whether intentional or unintentional, of exclusion, isolation, and marginalization can be argued as tools to control, ultimately acculturating the bodies of the underrepresented faculty and staff, by shaping their overall experience in several university spaces. I argue that acculturation is a problematic form of cultural taxation as it negates diversity efforts. While those particular minority members are physically present in their institutions, they are not contributing to cultural diversity. Adopting another culture recuses the presence 
of one's own. These actions are counterproductive and inhibit the cultural diversity of the academy.

Participating in diversity-related matters while attempting to complete tasks related to promotion and tenure effectively has the potential to lead to over-commitment and burnout (Mahoney et al., 2008, p. 785). Committing oneself to service and diversityrelated matters is not always something that faculty of color are forced to do. Shavers, Butler, and Moore (2014) indicate that faculty of color (but more specifically in their work, Black) often express a desire to engage in those opportunities. However, because of the underrepresentation of faculty of their engagement can quickly, yet subtly or drastically shift into over-commitment.

Feelings of isolation also surfaced in the literature as a reoccurring theme. Underrepresented minority faculty reported a sense of not belonging. This conception developed from feelings of exclusion, invisibility, isolation, being of poor fit, and the fact that there were, typically, not many other members that looked like them in their departments. These circumstances can be attributed to the impact of stereotypes, which like a domino effect, can impact URM faculty opportunities for collaboration and publications with non-URM faculty, which consequently impact promotion and tenure (Rodríguez et al., 2015). Trower (2003) attribute "lack of colleagueship, networks, and mentors" (p.6) to isolation.

Othering, which entails viewing one's ethnic group as superior and ultimately the standard to judge others by, contributes to isolation. Othering triggers "social isolation and exclusion from decision-making processes" (Hassouneh et al., 2014, p. 6). Minority faculty isolation occurs innately as a result of their low numbers, and othering amplifies 
the isolation and contributes to marginalization and loneliness (Hassouneh et al., 2014). Isolation can be a form of cultural taxation in relation to what it takes to survive and navigate academe as a minority; Withstanding and growing within an organization despite isolation.

Parts of the interview protocol was designed to indirectly address these aforementioned concerns; however, unlike the data gathered from the literature presented prior to the study, which were focused on faculty of color in predominately White spaces, the study applies these concerns to underrepresented faculty and administrators in a multicultural space.

\section{Consequences of Cultural Taxation}

Diversity-related efforts of URM faculty do not carry much weight in some institutions, and consequently, do not serve as a significant contributing factor to promotion and tenure. As referenced by Blackburn and Lawrence (1995), faculty that have heftier service loads risk facing a backlash in the tenure process. The time dedicated to improving or contributing to diversity within their respective institution takes away from the time available to focus on matters that are otherwise valued and contributory to the advancement of their status at the institution (Rodríguez et al., 2015; Mahoney et al., 2008; Syed et al., 2018; Turner \& Gonzalez, 2008; Blackburn \& Lawrence, 1995). Faculty of color (and women) tend to have heavier teaching and service loads than their White counterparts (Trower, 2008, p. 2). The circumstances aforementioned are results of lack of faculty of color, the obligatory pressure to advise minority students and serve on multiple committees, and intentionality - as for some faculty of color enter their positions with the intentions of serving in the ways mentioned (Trower, 2008, p. 2). 
Minority faculty can also experience a sense of responsibility to serve their respective communities, which is not always conducive to their career advancement because of the time it negates from their prescribed duties and the devaluation of their efforts in the academe. Trower (2003) argues for the monitoring of teaching and service loads to ensure equity during evaluations for tenure. Some FOC experience the discrediting of their research, especially when it is related to minority-related issues which creates a struggle to balance a moral obligation with an effort to progress professionally (Pololi et al., 2010, p. 1366). Minority taxation creates a barrier to success for underrepresented minority faculty members. Isolation, too, affects promotion and tenure (Trower, 2003, p.6). Underrepresented minority faculty that have "extra" responsibilities are more likely to be found in the lowest paying ranks (Rodríguez et al., 2015, p. 3).

Prior studies address the experiences of faculty, ultimately neglecting the experiences of administrators who are "the key to the success and quality of their institutions" (Austin, 1984, p.3). The focus on faculty in previous studies privileges their importance in academic spaces, which warranted the investigation of their experiences regarding cultural taxation. Recognizing that administrators "are increasingly entering into domains that were once the primary responsibility of academics" (Bassnett, 2005, p. 101) presents a need to investigate their experiences. Also noteworthy is the fact that "the decisions they make and the manner in which they make and implement policies determine not only the daily operations of a college or university but also its future" (Austin, 1984, p.3). 


\section{Cultural Taxation and Critical Agency}

Critical agency is "the ability to critically assess and, where necessary, reject, existing ... norms and values” (Poveda \& Roberts, 2017, p. 121). Researchers address "justice in the academy" and challenge the problematizing of service (cultural taxation). Service can function as "the stage for critical agency" (Baez, 2000, p. 387). The notion of aligning cultural taxation and critical agency avoids the minimization of the exploitation FOC face, while reshaping the perception, and highlighting the importance of service within academe.

Studies recognize that some individuals have a desire to serve on diversity-related committees or assignments (Chang, Martinez \& Welton, 2016). Their participation is perceived as a form of self-fulfillment and an effort to practice critical agency to aid communities of color. While some individuals describe their desire to serve and give back to their communities, they remain intentional about not letting their institutions marginalize them by using them as tokens for the institution or becoming a "victim" of cultural taxation (Chang et al., 2016).

Though there is a desire to serve, there is an equal desire not to be taken advantage of; this sense of abuse as recounted by one URM faculty member "can be excessive at times, and that can be the tipping point when it is too much" (Chang, Martinez \& Welton, 2016, p. 707). Additionally, some participate in "culturally taxing" activities as a result of values; "Values centered on benefitting others, such as community uplift and education for social change" (Blake, 2018, p. 14). Understanding cultural taxation as a form of critical agency aids in understanding how faculty and administrators may make alternative meaning of their experiences regarding the phenomenon; it leans 
away from the victimizing concept and places a positive outlook on the activities associated with cultural taxation.

\section{Brief Overview of Hispanic-Serving Institutions}

There are a series of understandings of what defines a Hispanic-Serving Institution (HSI). HSIs can be broadly understood as colleges and universities that serve a significant number of Hispanic/LatinX students. Since its original definition in the Higher Education Act of 1965, the definition of an HSI has evolved, legally, to be defined as an institution of higher education that is accredited, with "an enrollment of undergraduate full-time equivalent students that is at least 25 percent Hispanic students" (Department of Education, 2019). There has been an increase in the number of HSIs, which can be attributed to "social, political, economic, and demographic factors" (Laden, 2004). The Hispanic Association of Colleges and Universities (HACU), was founded in 1986 and currently "represents more than 470 colleges and universities committed to Hispanic higher education success in the U.S., Puerto Rico and Spain" (HACU.net). The Hispanic Association of Colleges and Universities is responsible for the formal recognition of HSIs, which allowed the institutions to acquire federal appropriations. The organization has played a pivotal role in the advancement and recognition of HSIs.

\section{Cultural Diversity in the Workplace}

Green et al. (2002) define diversity as "acknowledging, understanding, accepting, valuing, and celebrating differences among people." This definition alludes to the understanding that the mere presence of people with differences does not indicate diversity. Green et al. (2002) also note that "managing diversity involves a departure from collective views of groups of people to valuing individual differences and talent in 
the workplace" (p.2); this understanding further emphasizes the complexity of diversity in the workplace and argues against oversimplified approaches to cultural integration. Kundu (2001) suggests that spaces that are occupied by individuals from different cultures should promote and practice incorporating the multitude of values into all aspects of its system.

There have been efforts, to include the implementation of federal laws, to promote diversity and protect underrepresented groups from workplace discrimination on the premise of race, sex, religion, and country of origin, which has contributed to the formation of cross-cultural workspaces. Cross-cultural workspaces can be understood as a complex space with several variables that can influence the experiences of its inhabitants. Within these spaces, however, lies the potential for what Kondrad et al. (2005) refer to as asymmetric experiences. The asymmetric experiences are founded in under, and overrepresentation as the minorities are often tasked with having to behave in certain ways in order to "overcome the social discomfort of difference" (Konrad et al., 2005 , p. 15) as it relates to the dominant group. Also, although there have been efforts made to promote diversity within organizations, certain subpopulations remain underrepresented in spaces where top-decisions are made in the workplace (Grimson, 2018). Konrad (2005) emphasizes the power/dominance dynamics in the workplace and how it should not be ignored in the exploration of workplace diversity. Jacques (1997) notes that ignoring power/dominance dynamics in relation to workplace diversity ignores issues that may be considered delicate, to include racism, which could put the legitimacy of the individuals that hold power within respective organizations into question. 
The power/dominance dynamic is particularly important in understanding dynamics within FIU because its demographic is composed of, predominantly, minorities; however, there is the presence of a dominant minority group; this releases the institution from the normative barriers that present issues of diversity as a predominately White, non-Hispanic vs. "others" matter. The perception of social norms can also impact the behaviors of individuals in cross-cultural spaces. Chen (2010) presents data that shows how social norms influence discrimination among minorities. Harbi (2016) presents that "minorities widely share prejudice opinions towards other minority groups.

Cultural diversity in the workplace can have positive and negative effects. Positive effects include but are not limited to creating a demographic that can integrate with other diverse entities beyond the workplace, innovation, varied perspectives, and increased creativity. Negative effects include but are not limited to the potential for communication inaccuracies, social and collaborative work barriers, and "dysfunctional adaptation barriers" (Martin, 2014). Cultural diversity has been shown to decrease social integration (Stahl et al., 2010), while social integration has been linked to workplace satisfaction, and reduced turnover (O’Reilly et al., 1989; Stahl et al., 2010); and poor social integration has been shown to impact workplace satisfaction and turnover negatively.

Martin (2014) attributes the effects of cultural diversity in the workplace to how well organizational leaders manage them. Cox (1991) describes three ways that organizations implement diversity: monolithically, pluralistically, and multiculturally. Cox argues that a monolithic organization's implementation of diversity involves merely the existence of an affirmative action plan. Plural organizations engage in more 
aggressive recruitment and promotion of underrepresented individuals; however, those underrepresented individuals are expected to assimilate into the culture of the dominant group. Multicultural organizations are argued as ideal as they provide a space where differences are valued.

Social interactions, employee performance, and productivity can be influenced by cultural diversity (Brislin, 2008). Diverse workforces have been shown to perform better than more homogenous workforces (Herring, 2009); however, failure to address internal issues related to diversity can negatively impact the institution (Scarborough, 2019).

\section{Language and Power}

Within FIU, an HSI, Spanish is a common language among many of the students, faculty, and administrators. De Kadt (1993) argues that language, in general, does not have power, but that the power of a language is tied to space and time. She states that:

Specific languages, located in specific societal contexts, which exercise power, a power, however, which is largely a function of the particular roles these languages fulfill in 'their' society: a language mediates the power relations pertaining to its societal context (p.4).

Stalin (2008) explains that language has the ability to serve as a stabilizing force and that its usage has the ability to also serve as a "tremendous power of resistance to forcible assimilation" (p. 24). While language can be used to combat external cultural pressures, language can also be used as a categorizer that indicates who will be on the receiving end of potentially discriminatory behaviors. Ogbonna \& Harris (2006) describe how discrimination could be determined by linguistic deficiencies or differences. In some cases, the mere presupposition that an individual cannot speak a dominant language can make one susceptible to prejudicial treatment (Van Laer \&Janssens, 2011). 
Marschman-Piekkari (1999) explores the impact of language on communication within large multicultural organizations and how language has the power to impact daily operations. Despite linguistic differences, openness to linguistic diversity has been shown to have positive impacts on group performance and group satisfaction (Lauring \& Selmer, 2011, p.88). Despite ones openness to linguistic diversity and willingness to learn another language, studies have shown that adults have the tendency to attain lower levels of proficiency during second-language accusation than younger acquires that may have spent years learning and utilizing multiple languages (Collier, 1987; Dulay \& Burt,1978; Krashen, 1982).

\section{Oppression in the Workplace}

Markert (2010) discusses the changing face of racial discrimination as Hispanics became the dominant minority in the United States using the power-threat theory. $\mathrm{He}$ dissects and explains how the increase in the Hispanic/LatinX population in the U.S. contributed to increased levels of discrimination towards them because, as the powerthreat theory suggests, the larger the minority group becomes, the more of a threat they become to the dominant group. In turn, the dominant group flexes its power and authority to institute legal and alternative measures to protect their dominant status. Members of the Hispanic/LatinX community have historically been subjected to oppression in the United States, and in recent times, the degree of oppression has increased. Freire explains that "the oppressed, instead of striving for liberation, tend themselves to become oppressors, or "sub-oppressors" (p. 45) and that "In their alienation, the oppressed want at any cost to resemble the oppressors, to imitate them, to follow them" (p. 62). 
Alleyne (2005) provides an argument about workplace oppression in which she uses the phrase to describe "complex organizational dynamics and silent forces that give rise to difficulties involving issues of power and powerlessness and of the dominant and dominated" (p. 287)

Young (2013), in the context of discussing oppression in the cultural imperialism promoted by nation-states, nevertheless presents a lens by which one can understand the macro- and micro-oppressions that take place in social institutions, especially those that link more directly to the nation-state, such as universities. According to Young, the following "faces" of oppression: exploitation, marginalization, powerlessness, cultural imperialism, and violence. Young (2013) defines exploitation as "The act of using people's labors to produce profit while not compensating them fairly" (p. 1), marginalization as a form of oppression which is presented as "a process of exclusion" (p. 2), powerlessness as being associated with the "inhibition to develop one's capacities, lack of decision making power, and exposure to disrespectful treatment because of the lowered status" (p. 2), cultural imperialism as "taking the culture of the ruling class and establishing it as the norm (p. 3), and violence as "attacks" that can "damage, humiliate, or destroy" a person (p. 4). Again, even though Young was explaining larger social forces, I argue that, on the basis of data provided by the participants, Young's (2013) five faces of oppression can be used as a framework to understand workplace oppression.

\section{Chapter Summary}

This chapter provides a review of literature that is relevant to the study. I highlight the democratization of higher education and how it played a role in the degree of diversity in institutions today and how that could contribute to the presence of cultural 
taxation within the academy. As a recap, cultural taxation is a phenomenon experienced by minorities predominately, but not exclusively. As long as one has a commitment to diversity-related matters, they too can fall "victim" to cultural taxation. However, research does argue against the victimizing concept of the phenomenon; in fact, cultural taxation can be empowering if understood through the lenses of critical agency and making changes within academia.

In contrast, the phenomenon can also be understood as a form of oppression. Progress in the diversification of members of the academy in higher education can potentially lead to the reduction of the phenomenon in higher education as the increase in diversity can potentially lead to the normalization of "others" in particular spaces and hence reduce the pressures and taxing of those few underrepresented member of academe. Florida International University has been legally classified as an HSI for several years. HSIs have served as an educational advancement tool for many Hispanic and LatinX students and continue to contribute to educational access and achievement. Despite how cultural taxation is understood, its overarching understanding, as presented in prior literature, is heavily aligned with faculty of color in predominately White spaces. The significance of FIU and other MSI's with regards to their role in promoting minority success further lends itself to the importance of the study. Understanding the experiences of the faculty and administrators that occupy these spaces is important because although some people understand acts associated with cultural taxation as fulfilling, cultural taxation has been noted to have an adverse influence on those who experience it which has caused, in some instances, employees to leave an institution due to reduced job satisfaction, feelings of isolation and burnout. 
The study sought to contribute to the literature about cultural taxation, thereby aiming to expand the understanding of the term outside of faculty of color in predominately White institutions to cultural taxation in any space, to include MSIs where one would be classified as underrepresented among a dominant group. In doing so, I provide alternative understandings of the phenomenon and the environments that induce it. The next chapter will address how I conducted the study and why I chose this specific methodology. 


\section{CHAPTER III}

\section{METHODS}

The study used a qualitative interview approach. The aim was to explore the experiences of underrepresented faculty and university administrators as it relates to cultural taxation in a setting where minorities predominate the population. During a qualitative interview, "respondents are asked carefully crafted questions but are free to answer them in their own words rather than required simply to choose one or another predetermined alternative" (Weiss, 1994, p.12).

When the study was proposed, it was titled: The Experiences of Underrepresented Faculty and Administrators: A Study of Cultural Commitment and Obligation. The foundational definition of cultural taxation inspired the original title as it related to the obligation to show good citizenship towards an institution, and demonstrating commitment to a cultural group. I altered the title to conceal the actual intentions of the study in an effort to minimize the chances of influencing the participants understanding of their experiences, given the potential negative perception of the term "taxation." Each participant was informed of the study's true intentions following their interview - the revelation of the study's intentions, in some cases, led to the divulgence of more data from some of the participants.

The study brought a theoretical construct to the foundation. The interview questions were framed using the construct, and the analysis was done by examining how the informants address the construct during the interviews (Elmore et al., 2006, p. 360). The theoretical construct for the study was cultural taxation. As a result, part of the study explored the experiences of the participants using questions framed around the published 
understanding of cultural taxation. To capture any component that may not have been addressed, and to apply a more constructivists approach, the study also includes broad questions in which the participants were able to construct responses from raw experiences.

The methodology was selected to shed light on the experiences of underrepresented faculty and university administrators, providing a space for the participants to share their realities. Because the study was not interested in the holistic experiences of underrepresented faculty and administrators, a qualitative interview study was appropriate because "in the qualitative interview... the interviewer, as a representative of the study, is responsible for directing the respondent to the topics that matter to the study" (Weiss, 1994, p. 8); This approach allowed me to access specific components of their experiences as it relates to the theoretical construct attached to the research question. By framing the questions around the published components of cultural taxation, I was able to narrow the focus of their experiences. "In general, the dense information obtained in qualitative interviewing permits description of the many sectors of a complex entity and how they go together" (Weiss, 1994, p.10).

I guided the direction of the study as the interview questions were semi-structured and constructed in a way to gain specific aspects of the participants' experiences in an interminority setting as it relates to the specific phenomenon-cultural taxation. The philosophical assumption guiding the study was constructivism, which is geared towards understanding the subjective meanings of experiences (Creswell, 2018, p. 24). 


\section{Piloting the Study}

As expressed by Connelly (2008), a pilot study is a trial run of a proposed study that utilizes a small sample before the study is applied to a larger scale study. Pilot studies have several purposes to include "developing and testing the adequacy of research instruments, assessing the feasibility of a full study, designing and testing the protocols for the larger study, establishing and testing the sampling and recruitment strategies, collecting preliminary data, obtaining effect size information, and training research assistants" (Connelly, 2008, p. 411).

The main purpose of the pilot study was to test the interview protocol, gauge if the questions were clear, and gain insight on how long the interviews may take (while fully understanding that time would vary depending on individual experiences). The trail allowed me to evaluate the flow of the interview, which later informed my decision to make adjustments to the study's interview process approach.

The pilot study I conducted took place at FIU with an underrepresented student employee. I selected this participant because of his role within the institution, his demographic status, and because I wanted to avoid running a trail of the study with someone who could potentially serve as an actual participant. The participant was a Black male originally from St. Lucia. He was employed in a role that allowed him to work with multiple levels of administration within the institution; he also had a supervisory role over other student employees. His placement was reminiscent of a microcosm of a university administrator but on a student scale. I informally invited the student to participate in the study and treated him as though I were interacting with university 
faculty/staff. I did not record the session, but I did time the session and noted any concerns that I had as it related to my questions.

Following the pilot, I found that my interview protocol, without adequate followup, could lead to the abstraction of potentially low-quality data. In many instances, I had to probe further to obtain data rich responses from the participant. I had conceptualized a different outcome with regard to how an interview would flow given the questions I designed, however, what I had expected was not the case during the pilot run. As a result, I decided to allow my original interview protocol to serve as a guideline to a less structured dialogue, which was determined to better serve study, by providing an opportunity to capture a better quality and quantity of data. I will now continue to describe to specifics of the actual study.

\section{Setting}

The institution utilized for the study was Florida International University (FIU), Modesto Maidique Campus (MMC), which is classified as a large, public, four-year, Hispanic-Serving Institution (HSI) in the southeast region of the United States with a majority-minority faculty \& staff makeup. The qualitative interview study focused on underrepresented faculty and administrators as they relate to FIU's demographics. The demographic makeup of FIU provided an opportunity to explore racial, cultural, and gender-related dynamics that have been absent from prior literature about cultural taxation. The setting allowed for the exploration of the experiences of underrepresented faculty and administrators in a majority-minority environment. 
I chose to focus on FIU for two main reasons. 1. The faculty and administration demographic composition, which consists of, predominately, members that would be classified as minorities - within the demographic makeup are subgroups of minorities (77\% minority makeup). 2 . My proximity to the faculty and university administrators, as well as some of the professional relationships I have developed. The setting and demographics create an environment that induces cultural taxation from internal, external, and social forces, which ultimately led to what I fleshed out as four types of cultural taxation (or costs).

\section{Participants}

The participants for the present study were those who qualify as underrepresented faculty and university administrators at the HSI as they relate to the overall demographic makeup of the university, and their respective spaces. I was specific to the total overall demographic makeup because, as presented in the data (see charts below), Whites predominate the faculty count; however, when accounting for faculty and administrators collectively, they do not. Please note that the data presented in the first subsequent is reflective of the demographic count at the time the data for the study was collected. The second chart is reflective of the most recent available data. 


\begin{tabular}{|c|c|c|c|c|c|}
\hline \multicolumn{2}{|c|}{ Faculty Count $\downarrow$} & \multicolumn{2}{|c|}{ Staff Count $\downarrow$} & \multicolumn{2}{|c|}{ Total Demographic Count $\downarrow$} \\
\hline White & 1,129 & Hispanic/Latino & 4,138 & Hispanic/Latino & 4,856 \\
\hline Hispanic/Latino & 718 & White & 1,061 & White & 2,190 \\
\hline Asian & 241 & $\begin{array}{l}\text { Black or African } \\
\text { American }\end{array}$ & 924 & $\begin{array}{l}\text { Black or } \\
\text { African } \\
\text { American }\end{array}$ & 1,114 \\
\hline Not Reported & 191 & Not Reported & 789 & Not Reported & 980 \\
\hline $\begin{array}{l}\text { Black or African } \\
\text { American }\end{array}$ & 190 & Asian & 235 & Asian & 476 \\
\hline $\begin{array}{l}\text { Two or More } \\
\text { Races }\end{array}$ & 18 & $\begin{array}{l}\text { Two or More } \\
\text { Races }\end{array}$ & 82 & $\begin{array}{l}\text { Two or More } \\
\text { Races }\end{array}$ & 100 \\
\hline Pacific Islander & 2 & Pacific Islander & 3 & Pacific Islander & 5 \\
\hline
\end{tabular}

Data provided by https://accountability.fiu.edu/ - Reflects Data from 2018

\begin{tabular}{|l|r|l|r|l|r|}
\hline \multicolumn{2}{|c|}{ Faculty Count } & \multicolumn{2}{c|}{ Staff Count $\downarrow$} & \multicolumn{2}{c|}{$\begin{array}{c}\text { Total Demographic } \\
\text { Count } \downarrow\end{array}$} \\
\hline White & 1,092 & Hispanic/Latino & 4,251 & Hispanic/Latino & $\mathbf{4 , 9 8 9}$ \\
\hline Hispanic/Latino & 738 & White & 1,040 & White & $\mathbf{2 , 1 3 2}$ \\
\hline Asian & 233 & $\begin{array}{l}\text { Black or African } \\
\text { American }\end{array}$ & 924 & $\begin{array}{l}\text { Black or } \\
\text { African } \\
\text { American }\end{array}$ & $\mathbf{1 , 0 9 9}$ \\
\hline Not Reported & 193 & Not Reported & 843 & Not Reported & $\mathbf{1 , 0 3 6}$ \\
\hline $\begin{array}{l}\text { Black or African } \\
\text { American }\end{array}$ & 175 & Asian & 246 & Asian & $\mathbf{4 7 9}$ \\
\hline $\begin{array}{l}\text { Two or More } \\
\text { Races }\end{array}$ & 21 & $\begin{array}{l}\text { Two or More } \\
\text { Races }\end{array}$ & 83 & $\begin{array}{l}\text { Two or More } \\
\text { Races }\end{array}$ & $\mathbf{1 0 4}$ \\
\hline Pacific Islander & 11 & Pacific Islander & 17 & Pacific Islander & $\mathbf{2 8}$ \\
\hline
\end{tabular}

Data provided by https://accountability.fiu.edu/ - Reflects Data from 2019

The roles of faculty and administrators intertwine. - "Guaranteeing educational quality and efficient procedures means that academics and administrators must work more closely together" (Bassnett, 2005, p. 101); this warranted the inclusion of both roles in the study in contrast to the focus on faculty in prior literature. The motivation behind focusing on these underrepresented groups, specifically, was Amado Padilla's original reference to the impositions placed upon individuals as a result of their racial, ethnic, or presumed knowledge of cultural differences; differences being the key term. The targeted 
group was representative of populations that are "different" from the majority group at the institution and within the spaces they occupy.

The study made one exception to the participant makeup, and that is to members of the Afro-LatinX community; that is, for those who identify as Black. The exception is a result of the racial heterogeneity of the Hispanic/LatinX community. It was important to ask how the participants self-identified in an effort to avoid making assumptions about their ethnic identities. "Afro-Latinos often adopt the customs and culture of Latinos, [but] they exhibit physical characteristics that are typically associated with African ancestry and, hence, are categorized as Black" (Fernandez, 1996). Comas-Diaz (1996) explains the polarization of racial identification, which requires Afro-Latinos to define themselves as Black or White. Members of the LatinX community face being classified/identified with regard to their racial and ethnic backgrounds. As a result of this conflation of race and ethnicity, I opened the study up to those who identify as Afro-LatinX/Black.

With regards to faculty, my study was open to any underrepresented faculty member regardless of their employment status (i.e., full-time or part-time), tenure status (multi-year; non-tenured, not tenure-earning; not-tenured, tenure-earning; not tenureeligible; or tenured), rank or instructional status which allowed me to gain diverse perspectives and gauge different experiences. I had opened the study to part-time employees because I felt they could, too, contribute some insight regarding the phenomenon, however, all of my participants, both faculty and administrators were classified as full-time. As it relates to faculty, some were tenured while others were nontenured. Administrative levels, also varied within the sample although most were managers and above with the exception of one coordinator. 
My original goal was to interview up to 20 faculty and administrators. I used purposeful and snowball sampling to obtain a total of 15 actual participants. As defined by Creswell (2018), purposeful sampling “intentionally [samples] a group of people that can best inform the researcher about the research problem under investigation" (p. 148), while snowball sampling "identifies cases of interest from people who know what cases are information rich" (p. 159). The use of snowball sampling was incorporated to aid the study by connecting me to other information-rich participants, and to help save time with recruitment.

As a four-year employee of the institution utilized in the study, I had already developed rapport with other FIU employees across campus. In addition, during, and leading up to the start of the study I had attended several campus events in order to expand my network. The expansion of my network allowed me to have developed prolonged engagement with some of the (at the time, potential) participants which aided in trust building as recommended by Creswell (2003). My approach in recruiting began by contacting the underrepresented minority faculty and university administrators that I was aware of. From there, I was able to inquire, through them, about other underrepresented faculty that I may be able to contact.

To guard any potential researcher biases, I was intentional about recruiting participants who appeared to be very enthusiastic about their work and presence on campus, not just individuals that appeared visually "taxed." I make that statement with full awareness of the fact that some faculty and administrators may not project their emotions on campus - with that, my ultimate goal was to attract a diverse group in an effort to obtain disconfirming cases. I focused on race and ethnicity as I sought to explore 
how cultural taxation may or may not exist at FIU; I was also intentional about sampling participants from various genders, understanding that these classifications, too, can be incorporated into the understanding of cultural taxation; however, more female candidates responded back to me expressing their willingness to participate in the study. Please see the next page for a chart presenting the participant demographics in the order the interviews occurred.

\begin{tabular}{|c|c|c|c|c|c|}
\hline Participant & Race & Ethnicity & Gender & Years & Position Type \\
& & & & at FIU & \\
\hline 1 & Black & Nigerian-American & Female & 5 & Faculty - Non-tenured \\
\hline 2 & Black & African-American & Male & 7 & Administrator \\
\hline 3 & White & European-American & Male & 29 & Faculty - Tenured \\
\hline 4 & Black & African-American & Male & 3 & Administrator \\
\hline 5 & Black & Haitian-American & Female & 6 & Administrator \\
\hline 6 & White & European-American & Female & 4 & Faculty - Non-tenured \\
\hline 7 & Black & Cuban-American & Female & 10 & Faculty - Tenured \\
\hline 8 & Multi- & Brazilian-American & Female & 7 & Faculty - Non-tenured \\
\hline 9 & Black & Bahamian-American & Female & 3.5 & Administrator \\
\hline 10 & White & European-American & Female & - & Administrator \\
\hline 11 & Black & Haitian-American & Female & 12 & Faculty - Non-tenured \\
\hline 12 & Black & Jamaican-American & Female & 33 & Faculty -Tenured \\
\hline 13 & Multi- & Iranian-American & Female & 10 & Faculty - Tenured \\
\hline 14 & Black & African-American & Male & 4 & Administrator \\
\hline 15 & Black & Trinidadian-American & Female & 5 & Administrator \\
\hline
\end{tabular}

Sandelowski (1995) suggests that a sample size of up to 10 is "judged adequate" for homogeneous populations; the homogeny of this sample lies in the participants' underrepresented status on campus as it relates to the campus demographics. As a result, I aimed to interview up to 10 faculty members and up to 10 administrators, but I ended up successfully recruiting 8 faculty and 7 administrators. The participants must have been a part of the underrepresented group within the institution, which is predominately Hispanic/LatinX. The qualifiers were different from what qualifies one's minority status, 
according to the United States Census. One's status as underrepresented was determined by the parameters of the institutions' racial/ethnic makeup.

In a qualitative study, the "sample size has to be representative of the population under consideration as a breadth of inquiry is anticipated" (Boddy, 2016, p. p. 429). A representative sample is defined as "people who, taken together, display what happens within a population affected by a situation or event" (Weiss, 1994, p.17). For that reason, I aimed to recruit at least one representative from each underrepresented group to make up the sample to include: White, Black or African American, Asian, Two or More Races and Pacific Islander - this was because, "Individual cases can also provide a new, deep and nuanced understanding of previously unexplored phenomena" (Boddy, 2016, p. 428). I was unable to recruit a representative from every underrepresented group, but I was able to recruit a diverse sample. The group that was excluded from the study were those that are classified as "Not reported" because there is no indication of which defined group they would fall under, and the study is exploring defined groups.

\section{Data Collection}

Semi-structured, open-ended, interviews were the sole instrument for gathering data. Qualitative research interviews "[attempt] to understand the world from the subjects' point of view, to unfold the meaning of their experience, to uncover their lived world" (Kvale, 1996, p. 482). I utilized an interview guide, which is "a listing of areas to be covered in the interview along with, for each area, a listing of topics or questions that together will suggest lines of inquiry" (Weiss, 1994, p. 48). (See Appendix A). Interviews took place in the participants' setting of choice. The interviews were all conducted face to face/in-person so that I could observe any non-verbal gestures (e.g., facial expression). 
The participants' physical ques were documented in my notes during the interview sessions.

The study utilized an interview scheme that incorporated carefully orchestrated questions that indirectly addressed the published components of cultural taxation. This was done to avoid influencing participant responses because the term cultural taxation could carry a negative connotation. The interview protocol included both general and focused questions about the participants' experiences. The general questions were designed in order to gauge any aspects that may have been neglected in the focused questions that address the published components of cultural taxation as the term continues to grow and evolve.

Also included with the interview protocol in appendix A is a mapping of what each question was intended to assess. The first line of questions highlighted in dark blue boxes were designed to initially engage the participants, get them comfortable speaking, gather background information about them, and gauge their perceptions about their environment (e.g. campus). The second line of questions highlighted in orange boxes were designed to explore the participants experiences as they relate to cultural taxation, as published in prior literature. The very first question in orange requested for the participants to recall any overall experiences they may have encountered that were directly related to cultural taxation - I was careful how I asked them about that particular aspect of their experience by avoiding the use of the term "cultural taxation," instead, I framed the question by incorporating the initial definition of the term that guided the study. The white boxes linked to the orange boxes indicate what component of the phenomenon the subsequent orange box was intended to explore. The question in the 
remaining purple, pink, green, light blue and grey boxes were broader questions designed to capture any component of their experiences that may not have been addressed in the prior questions that were designed to elicit experiences that were directly related to the phenomenon; those questions were included to allow participants to construct responses beyond the context of my initial questions. The responses provided by the participants prompted further probing that is not represented on the protocol as this varied across participant.

The goal was to gather detailed descriptions of the participants' experiences. These detailed accounts contribute to the study's transferability (Guba \& Lincoln, 1985) as generalizability is not the aim. The data for the study were gathered by way of one 60120-minute semi-structured interview per participant. The interviews averaged about an hour- some were longer than others depending on their experiences and recollections. Each interview took place at the participants' location of choice, many took place in their personal office if it allowed privacy. Others took place in rooms I reserved on campus. I started interviewing participants March 27, 2019 throughout the beginning of the summer. I had a steady flow of participant recruitment.

During the interview sessions, I used two recording devices (an actual recording device and the recording device embedded within my laptop) to capture every moment and ensure there is a backup device in the event that one malfunctioned. The questions were designed to elicit specific experiences pertaining to the components of cultural taxation, as recommended by Van Manen (2016). Following the initial questions, followed by additional probing, I introduced the term cultural taxation to the participants and explained that the topics addressed earlier are recognized as components of the 
phenomenon. This step, for some, extended the interviews as they had more to expel following their introduction to the term and its meaning.

\section{Data Analysis}

Following each interview, the data were transcribed using two services to produce the transcripts in a time-efficient manner: Rev and Otter, which are online transcription services. I switched to the latter because of financial cost. The interview recordings were typically sent for transcription immediately after the interviews were conducted; I wanted to keep a steady cycle of information and review the transcripts while the information was fresh in my mind. I made a practice of formulating participant descriptions immediately after each interview to include behaviors, trends, and personal information to the extent that I could still maintain a degree of anonymity. Upon receiving the transcripts, I reviewed them at least three times to ensure the integrity and accuracy of the transcription and to better familiarize myself with the data before proceeding to analyze them for themes. Some had to be reviewed additional times because of the quality of the recording, which was often linked to the low volume of some of the participants' voices. I used Nvivo data software to organize and analyze my data. The Nvivo software is a qualitative data analysis package that is useful for research that is text-rich. The data were validated before and after it was uploaded into the Nvivo software to ensure that no data were lost or modified during the data transfer.

While reading the transcripts, I annotated them with any questions or comments that may have arisen, which were generally related to thematic possibilities, correlating statements across participants, and diction. Following these questions and comments, I used a hybrid coding system that incorporated a priori and in vivo coding. A priori codes 
are "codes set beforehand" (Elliott, 2018). Creswell (2013) notes that a priori coding "does serve to limit the analysis to the 'prefigured' codes rather than opening up the codes to reflect the view of participants in a traditional qualitative way" and suggests that "if a 'prefigured' coding scheme is used in analysis, [he] typically encourage the researchers to be open to additional codes emerging during the analysis" (p. 185). I used the pre-established forms of cultural taxation to apply to the participants' experience and coded the data using in vivo coding. In vivo coding was used to "keep the data rooted in the participant's own language" and "prioritize and honor the participant's voice" (Saldana, 2009). This allowed the codes to be participant inspired as well, rather than solely researcher generated, working from a constructionist standpoint. Following the in vivo coding, I conducted a second cycle of coding "to develop a sense of categorical, thematic, conceptual, and/or theoretical organization" (Saldana, 2009, p. 149) from the first cycle of coding to see if any new understandings of cultural taxation emerged.

I initially used Amado Padilla's "six identifiable forms" of cultural taxation as well as other "components" of the phenomenon, which were argued as such because of the time and energy the tasks negated from ones job duties as the foundation for my analysis. I combed through the data looking for statements that were related to the following: being called upon to serve as the "expert" on diversity matters, being called upon to educate members of a majority group about diversity, serving on affirmative action committees without any observable changes, serving as the liaison between the institution and the ethnic community even though one may not agree with the institutions' policies, taking responsibility for settling problems regarding sociocultural differences among the members of the institution, being called upon to serve as a 
translator or interpreter when the institution is presented with non-English speakers, irregular expectations, mentoring, coping with colleagues' problematic behaviors, differential legitimacy, and acculturation. I used Amado Padilla's "six identifiable forms" of cultural taxation as well as the other "components" as pre-established codes that I created preset nodes for within the Nvivo software. Nodes are folders created Nvivo to store data about specific themes that emerge in a study. When I came across statements that related to a specific pre-established code, I added the data to its corresponding node. While reading through the transcripts, I also came across other ways the participants experienced cultural taxation. When these instances surfaced, I created new nodes for them in Nvivo. From there I was able to add more data to the nodes that qualified under these new additional "ways" that cultural taxation can be experienced. I was able to add those emerging instances to what I had encountered in prior literature.

Codes were developed by transforming the participants' responses to ideas and then subsequently taking those ideas and applying them to all of the data pertaining to those particular ideas (Saldana, 2009). After my initial review of the data, I developed several themes; however, a significant number of them correlated with one another in ways that did not warrant their independent standing as themes. As a result, under the recommendation of my committee chair, I conducted an additional round of coding. In doing so, I was able to develop a series of three overarching themes and six subthemes that arose. In the study, the overarching "themes" revolved around the impositions of cultural taxation; these impositions are understood as pressures.

I reached those final themes by first reading through the transcripts several times and noting the participants' statements and abstractions made during their accounts to 
include events and encounters they have had on campus, emotions tied to their experiences, and in some case, stories about the experiences of other underrepresented faculty and/ or administrators that they were aware of. From the accounts provided by the participants, I was able to organize the data in accordance to the ways the participants experienced cultural taxation. From there, I reduced the data to conceptual, relationship, perspective, and setting codes (Vaismoradi et al., 2016), according to their experiences. The conceptual codes generally included the ways the participants experience cultural taxation. The relationship codes generally involved links between the participants' identities, positions on campus, and their experiences. The perspective codes generally pertained to how the participants' view and understand their experiences. The setting codes generally involved the participants understanding of the spaces they occupy. Amongst the aforementioned codes, I was able to further reduce the data to three themes and six sub-themes.

After organizing the data according to how the participants experienced cultural taxation, I went back through the data to further collapse the data into larger themes that their experiences were related to. From reviewing the participants' responses, I noticed that their experiences were related to the following: obligation, coping, service, expectations, integration, and tokenism - I recognize these as the subthemes in the study.

While reviewing the data, I noticed that participants' experiences were rooted in different forces. The categorical impositions (internal, external, and social) provide an understanding of where the phenomenon develops prior to becoming a part of one's experience - I recognize these as the overarching themes. Some of their experiences regarding cultural taxation were initiated by the institution, colleagues, and/ or students. I 
classify these as external impositions. The sub-themes that fell under this overarching theme were service, expectations and tokenism. Some of the participants' experiences were initiated by themselves; I classify these as internal impositions. The sub-themes that fell beneath this overarching theme were obligation (duty or commitment), coping, and critical agency. Other experiences relating to what I understand to be cultural taxation were initiated by the social climate/ environment; I classify these as social impositions. The sub-theme that fell under this overarching theme was integration. Please see appendix B for a visual layout of the study's themes, subthemes, and the ways the participants experienced cultural taxation.

I chose to focus on impositions as overarching themes because, as participants presented their experiences, the root of the pressures they experienced were distinct and worth categorizing in accordance with their origin to highlight the multiple forces present within the academy. Focusing on the impositions also serves to distinguish cultural taxation from only being a phenomenon that is inflicted upon underrepresented members of the academy by the administration; also arguing that while external and social impositions can be linked to under- and overrepresentation, some forms of cultural taxation can be linked to a sense of moral obligation.

As far as some of the statements presented in the succeeding sections, I constructed some of the more extensive statements by combining the participants' separate responses to a topic that were divulged at different points throughout their interview. In many cases, some participants addressed specific topics at different times throughout the interview session; I combined them into one statement, chronologically, to keep the data from singular participants together in order to maintain a degree of 
organization and to avoid quoting participants several times, about the same topic, separately, while capturing a holistic picture of the participants experience regarding the topic at hand.

During data analysis, when I encountered data I was unsure about, I reached out to one of my classmates for professional judgment. I chose this particular classmate because the individual is in the same stage as I am in the doctoral process and has focused much of his time and efforts on scholarship related to diversity and social issues; therefore I believed he could provide valuable feedback and clarity about some of the data that I may have been unsure about.

\section{Chapter Summary}

The purpose of this qualitative interview study is to expand the understanding of the cultural taxation to include the environment that induces its existence, how it is imposed, how it is experienced, and who can land on the receiving end. The study utilized an interview protocol that was designed to explore the experiences of underrepresented faculty and administrators as it relates to what I argue is cultural taxation, cultural taxation as published in prior literature, while also providing an opportunity for participants to construct other experiences that may have been absent from published literature; this has been mapped out in appendix A. I conducted a pilot of the study with a student that could be argued as, descriptively, a microcosmic representation of a university administrator to test the interview protocol, which allowed me to evaluate the flow of the interview which led me to make minor procedural adjustments. 
The study incorporates the experiences of 15 underrepresented faculty and administrators at Florida International University. This setting allowed for the exploration of an alternative demographic, unlike those previously explored regarding cultural taxation, while also allowing for the inclusion of populations that are often excluded from studies about underrepresentation because they fall into dominant categories (e.g. White faculty and administrators). The data provide by the participants in addition to a series of coding cycles led me to the three overarching themes (internal impositions, external impositions and social impositions) and six subthemes (service, expectations, tokenism, obligation, coping and integration). A mapping of the coding can be found in appendix B. The following chapter will provide the spatial context of the setting and introduce the participants. 


\section{CHAPTER IV}

\section{SPATIAL CONTEXT AND PARTICIPANTS}

This chapter presents the spatial context of the setting utilized for the study as well as descriptions of each of the fifteen faculty and administrators which were constructed from data gathered from the interviews conducted at Florida International University. The sample reflects participants from eleven colleges and thirteen different

departments. As prefaced, the study aimed to answer the following questions: 1 . To what extent do faculty and administrators experience cultural taxation at Florida International University, Modesto Maidique campus? 2. How do the experiences of faculty and administrators compare with regard to cultural taxation?

\section{Spatial Context}

In understanding the experiences presented in the study, it is essential to recognize the spatial context of the setting. Florida International University (FIU) was founded in 1965 and opened in 1972. In the spring of 1999, FIU received its HSI grantee status. Currently, FIU has a student demographic of 61\% Hispanic, 15\% White, nonHispanic, 13\% Black, 4\% Asian or Pacific Islander, and 7\% other minority groups. FIU is one institution - a space of its own. However, spaces tend to multiply (Barnett, 2010, p. 76), and within the university, each participant's experience varied based on their respective spaces and their understanding of the spaces they occupy. With regards to demographics, however, despite the demographic variances, all participants fell under underrepresented categories. Each participant shared their experiences with regard to multiple spaces on campus. Those covered in the study include workspace, academic space, and social spaces. I refer to workspace as spaces that link directly to 
work-related experiences; this would involve interactions between participants and other university personnel. Academic space refers to spaces that are primarily in relation to instruction, research, and/ or clinical activities. Social space refers to a less visible accumulation of relationships among members of the university at all levels to include students and personnel. While many of their experiences transcended and coexist across spaces, some experiences were reliant on specific spaces.

I believe it is also vital to warrant the significance of spatial context and understanding because the reader may not be in agreement with some of the statements made by some of the participants because their respective spaces do not confirm claims presented in this document so it may be challenging to conceptualize their experiences. One example would be references made by a participant about the absence of Blacks in senior-level positions, although I am aware of a few senior-level positions occupied by Blacks- Black women in particular. However, I argue that this does not negate what the participant observes and understands as a part of their lived experience due to the spaces they occupy and the absence of proximity to certain situations.

Furthermore, I provide the spatial context for Miami to ground the study and the experiences shared by the participants. Florida International University is located in Miami, Florida, and was articulated by some of the participants to be a "Mini-Miami." With that, I believe that it is important to divulge a bit of Miami's history as it relates to the dominant demographic as much of the experiences shared in the study are in relation to underrepresentation and/or dominant-dominated dynamic.

In the 1950s, Hispanics and members of the LatinX community settled in Miami in large populations. In 1959, Cuba underwent a change in leadership that altered its form 
of government (Skidmore et al. 2010). The new government was unfavorable to many Cubans and was especially unfavorable to those who identified as members of the middle and upper-economic social class. As a result, many departed from Cuba to the United States with support of the United States Government by way of the Cuban Readjustment Act of 1966, an opportunity that no other LatinX or Caribbean county had been granted. Today, according to the most recent data provided by the US Census Bureau, $72.2 \%$ of Miami's population is Hispanic or LatinX and largely Cuban.

Benson (2016) presented that Cubans in Miami historically maintained a degree of silence with regard to the needs of Afro-Cubans. He also highlighted that, for the most part, Cubans, collectively, were not very active with the social justice struggles in the United States in conjunction with having favored status with the U.S. government (p. 135)- referencing the 1960's era. Also noted was that because of the small numbers of Afro-Cubans that immigrated to Miami, there was not a tendency to not "acknowledge the demands of Afro-Cubans or work to integrate Blacks and Mulattos into their social spaces" (p. 135), which could be indicative of a racial dichotomy and lend itself to how these behaviors can transcend into the university space and perhaps provide some understanding of the participants experiences. In the next section I present the participant profiles in the order in which the interviews were conducted.

\section{Participant Descriptions}

Due to the potential sensitivity of some of the subjects divulged, and in some cases, the potential ease of identification based on their underrepresented status, I considered using pseudonyms; however, I wanted to distance the identity of the participants from the data as much as possible- this was done to aid in preventing the 
reader from being able to potentially construct participants identities by connecting their statements throughout this text. To protect my participants' identities, any quotes included in this document will be identified by race, gender, and position type (i.e., faculty or administrator), solely. I considered applying more detail to their identifiers by being more specific with their position tittles, but there were a few that were so specific that the inclusion of the position title would have put the participants' identity at risk of exposure. For example, one of my participants occupies a position on campus that is only held by only two people at the institution. Another participants' position title was directly connected to the office in which he/she occupied; therefore, I only used "faculty" or "administrator" to classify the participants' positions. This was done to focus on the data rather than the specific details of the source of the data. Moreover, again, I wanted to disconnect the data from the participants as much as possible, because some of the participants expressed fearing "backlash" as part of their experience during the study. Also, much of their descriptions exclude details of their work area and titles; this was also done to shield the participants' identities. The participant profiles ae presented in the order in which the interviews took place. I developed their descriptive profiles from data obtained from the interview transcripts, notes taken during the interviews, and memos I took throughout the study.

Nigerian-American Female Faculty

Participant one is a female, Nigerian faculty member who has a master's degree and two doctorate degrees. She was born and raised in a homogenous country (Nigeria), and as a result, she said that she does not, or may not see race the same as others stating that: 
I'm [pause] I have a racially homogeneous background. I never, ever experienced [racism] [pause] Well I know racism [pause] I didn't know when sometimes - [pause] like most Nigerians [pause] we are oblivious to it because we don't know it... You just know that this person did something that made you feel bad.

She prefaced her understanding of racial dynamics when she first came to the United States and how she developed her understanding with the help of a mentor at the university that worked directly with diversity initiatives stating: "She helped me understand. She helped me understand it. She started putting the world out there. She took me under her wing."

She has been working with FIU for about five years at the time of the interview. She also obtained her Master of Public Health and one of her doctorate degrees from FIU before her employment within the institution. She was delighted to participate in the study and was a pivotal part of my snowball sampling. She was very adamant about helping out a "little sister," which set the tone for most of the interview session.

During the interview, she expressed that she had a "unique position" and that her experience is likely different from many others; she stated this numerous times during the interview because while she was underrepresented in her department, she had access and connections to other nearby underrepresented female faculty across campus. She linked her overall experience as an underrepresented faculty member at FIU with her experience as an underrepresented student because she was a student at the institution first; her time as a student represents her initial exposure to the environment and set the foundation for her understanding of her underrepresented status on campus.

She attributed her positive experience to having a great boss and also working in an environment where there are other Black women nearby. However, in rating her 
feelings of integration and representation, she stated that within her department, she felt integrated at 10 . Within her respective college, that reduced to $7 / 10$ and as it relates to FIU overall, the rating fell to $5 / 10$. She used a rating system because she was having a difficult time articulating her experience with integration on campus. She was very critical about her understanding of racism, stating that "Sometimes, like most Nigerians, we are oblivious to it because we do not know it" and how another senior-level Black woman had helped her to understand and gauge it.

\section{African-American Male Administrator}

Participant two is an African-American male in an administrative position. He is a native of Miami and has been with FIU for seven years. He was more passionate in his expression of his experiences, but I attributed that to his frustration. He was very excited to participate in the study and often conflated his experiences at FIU with his experience as a Black man in the United States, but instead of being of being disadvantaged as a minority in a predominately White space, he felt that disadvantage in a predominately Hispanic/LatinX space stating:

So let's take it bigger, look at the university. I feel like there are opportunities for me. I try not to use any excuse but I don't feel like I have the same advantages as somebody with the last name Rodriguez... Perez, or you know?

His statement indicates that he believes his experience would be different if he was a member of the dominant group. Throughout this interview, the participant seemed to present a high level of frustration with the environment and his experience. I had to keep redirecting him back to focus on his experience at FIU because he kept leering away into his experiences overall as a Black man in the country. My actions were not done to 
discount what he was articulating to me but more so to follow-up with him about his accounts and how his experiences impacted him within FIU. I will qualify that he did say that FIU is a reflection of the country indicating that his experiences within FIU are similar to those he experiences generally, which was interesting because of the predominance of minorities within the institution versus the predominance of Whites within the United States, yet somehow there were perceived parallels as presented by the participant; he and others.

This participant was a little less critical of certain aspects and his evaluation of his experiences; His responses were also filled with anger, annoyance, and frustration. I could have potentially expected that because this participant was eager to participate in the study. It was almost as though his participation in the study was an outlet for him, especially since he did reference his perceived inability to express discontent in his workspace. It can almost be said that he invited himself to the study. He happened to ask me what my study was about during a casual hallway conversation and when I explained to him that it was about the experiences of underrepresented faculty and administrators, he volunteered himself, with much enthusiasm, to share his stories and experience as an underrepresented minority at FIU. I could have expected that that same excitement would have surfaced throughout his interview.

His body language throughout the interview was very relaxed at times, very slouchy, lacking energy, which kind of aligned with his frustration. He also referenced religion quite often regarding how he coped and why he relied on religion throughout his experiences at FIU. The participant is no longer with the institution; he accepted another 
position elsewhere. I have not followed up with him regarding his new place of employment.

\section{European -American Male Faculty}

Participant three is a White male who is not originally from Florida. His experience within the institution does not seem to have been seen through a lens of race. The participant expressed that he "[doesn't] feel underrepresented at all." He stated that he has never seen or understood himself as a minority; his inability to conceptualize himself as a minority was apparent throughout the interview, even though he subconsciously made statements that were reflective of an experience as a minority. During the interview he stated:

I never thought of myself as a minority before. And even though I've been here almost 30 years, I guess I kind of still don't think of it, but maybe for legal or demographic, statistical reasons. I have come around. And I do [pause] I do understand that I am a minority. But I don't have a problem with that. I don't think of myself as a minority. Even though statistically [pause] in this county [pause].

When he reflected on his experience, much of it was felt as a result of his sexuality, not race. Despite his unrepresented status; however, this individual expressed that he is adamant about making himself feel included even when those he attempts to socially integrate with on campus reject his inclusion. He has more of a forceful spirit in that he expressed not allowing himself to be excluded from any environment. His efforts include persistently placing himself in environments within his department and generally on campus despite the attitudes of those around him whose behaviors are dismissive, stating: "I never asked to be accepted. I'm going to be a part of the community, no matter what." 
His responses were all very brief. His experience seemed to have been removed from many forms of cultural taxation even though he mildly and unknowingly alluded to some components of the phenomenon. His idea of diversity also was grounded in his experience growing up in a mainly homogeneous region where he would have fallen into the classification of a member of a dominant group.

The participant seemed a bit filtered, especially with the briefness of his responses. I have known this participant for about three years at the time of the interview and have had many lengthy conversations with him in passing on campus. The individual is usually much more conversational, but in this instance, that claim did not hold until the conclusion of the interview, when the participant provided much more fluid and lengthy conversation. He was also politically correct in his responses and made quite a few disclaimers about his understanding of racial dynamics. I was unsure if this was due to a lack of trust with me as an interviewer or fear of falling into a stereotype as he once made the comment "I'm not one of the ones [pause]." He seemed adamant about presenting himself as a White male that understood some aspects of his perceived privilege. I say "some aspects" because quite a few of his responses could have been seen as carrying privilege.

Towards the end of the interview he said "I'm kind of boring for you. Sorry about that." He apologized and indicated that he might not have been very useful, but his inability to provide feedback about certain components of cultural taxation but his inability to produce feedback in some instances was data in itself. 


\section{African-American Male Administrator}

Participant four is an African-American male from northern Florida that has been with the institution for about three years at the time of the interview. He was in his last few months of employment with the institution; He had acquired a new position elsewhere, and his department was already made aware. With that, it can be assumed that there was a little fear of retaliation for sharing his experience even though the study is anonymous.

He was not very filtered throughout the interview; he did, however, maintain a high degree of professionalism. I've known this participant on a surface level for about nine years through a mutual friend; this, I believe, aided in the flow of the interview and degree of trust between the participant and myself. This interview was, however, one of the first times that I sat down with him and had an in-depth conversation about work-life at FIU.

The participant was very focused on not only his experience as an AfricanAmerican but also his experience as a male, the only male in his department. The intersectionality of him being a male and African-American played a significant role in his experience within the institution. He was also very focused on being able to differentiate the difference between workplace culture and the culture of the institution. During the interview he stated:

Yes, I understand it's very Hispanic-dominated, but at the same time, some people come from different backgrounds, and you really got to think about what culture you are trying to set. Are you trying to set a Hispanic culture? Are you trying to set a workplace culture? And that could be two different things. I think that's where FIU really needs to gear their attention towards, especially being an international university. 
He has a corporate background, so his understanding of a workplace environment is heavily aligned with the expectations of corporate America in predominately white spaces.

He seemed to have reflected on his existence in his own space on campus, heavily as many of his responses were well thought-out, well organized and presented assertively; I make this statement comparatively as some of the other participants appeared to need a bit more time to reflect and construct their responses. He, on the other hand, did not appear to have to dig deep to find and articulate his experience. He required less time to construct and relay his experiences and conceptualizations to me.

\section{Haitian-American Female Administrator}

Participant five is a Haitian-American woman who has been with the institution for about six years. She was very critical of her understanding of her position as underrepresented, what it meant to her, and what it requires of her. She was also in tune with the state of her mental health and what she needs to do for herself to maintain her peace of mind. I recall her mentioning that she has what she refers to as "a shield," which allows her to keep certain things out; she expressed that she can say no to requests as well.

She emphasized the overall lack of general Blackness in her department and within leadership overall in the institution stating that there are "Very few [Blacks]... [and] there should be more effort to recruit African descent people here in staff, faculty, everything." She presented a multi-tier understanding of her underrepresentation from the lenses of being Haitian-American and the lenses of being Black in general. She has a corporate background, and that does impact her lenses and how she views the institution. 
Additionally, the workforce demographic seemed to resonate with her heavily. Much of what she expressed during the interview regarding her experience within the institution was based on her underrepresented status, and the acknowledgment of it; it is something that seems to be a constant reminder to her. Throughout the interview she made several references about her perception of diversity within the institution. At some point she said: "As an employee, I feel like there's definitely a lack of promotion for the AfricanAmerican or blacks in this university." In another instance she said: "Here, like I said, more Hispanics are hired than blacks, and I think it's very uneven. It's extremely uneven, you know?" At some point she stated: "I... feel we need to do more work to kind of bring more equality, or at least to include more blacks within the employment, the workforce at FIU, if you will," and again:

There's definitely a need to have more blacks to be in positions here, all level positions, like management. Particularly management, but also other administrative staff kind of work, because really you can count on almost like your 10 fingers in each department how many blacks are there.

The previous statements were made at several different points during the interview.

She had conceptualized the study to be about discrimination, which could be an indicator that she subconsciously correlated underrepresentation with discrimination; this could also explain why discrimination was the lenses that she perceived much of her experience through.

\section{European-American Female Faculty}

Participant six is a White woman from the northeast region of the United States. She has been working with the institution as a faculty member since 2015 and has been a resident in Miami for twenty-five years. She was very reflective of her experiences. Her 
experiences as a White woman was heavily intersected with her experience as an individual with a physical disability.

Within her respective space, she expressed that she does feel like a minority. She refers to the notion of "clubs" and "inner circles," how she does not feel like a part of them, and that she feels like her experience would be much different if she were a member of the dominant group (e.g. Hispanic/LatinX) in her department. She even expressed that her experience as a disabled woman may even be different if she was not an "outsider" (i.e., a White, non-Hispanic woman) because if she were a part of the dominant group, perhaps they would care. In one instance she shared the following stories:

I found doing the [special event] very frustrating as somebody with a disability that ... the photographer said that I can't get down in the front row with the [rest of the] faculty. That I have to go up at the top with the students because I was [specifies disability]. And I thought the appropriate thing would be to have the pictures taken in a place that's accessible to me. But I did manage to [transport] myself down through grass to the front row. Again, [in another instance] when we took pics for the department. It was along a bunch of steps and there were people sitting in the front, and there was another line and another line and I can only be on the top. And I'm pictured on the top on the side, so it's just a very odd, inappropriate picture for a department. For me, I felt very [pause] it just was not a comfortable picture or depiction of the department because it just looks like, I'm literally [depicts disability], but if I was sitting I should be with everybody else who was sitting and the photographers weren't properly aware of how to do a picture appropriately, or maybe there were just kind of lazy or taking short cuts. [No one]... the chair of the department [pause] When I tried to suggest that we do this so we're all sitting together, it really got nowhere. I think if I was more a part of the inner circle, I would've been more accommodated.

Her observations during her time in her department led her to believe that if she was a member of the dominant group, which is Hispanic/LatinX, her situations would have played out differently. She was very clear in the processing of her experience; she even 
took notes to ensure she was addressing all parts of the study. She expressed frustration with her experience within the university.

The topic of language and exclusion reoccurred throughout the interview. She cannot speak nor understand Spanish. I was able to witness her being excluded from a conversation among other faculty, personally. I observed this weeks after our interview. Seeing how casual this took place made me understand a little better why language and exclusion were so prominent in her responses. It was as if she did not exist any longer, coupled with her perceived invisibility due to her disability.

After the interview, the participant mentioned that she had not viewed life through the lenses of the type of questions I was asking and that it allowed her to bring her experience and understanding to the surface. She stated that she tries not to allow things that are out of her control to bother her; she seemed to present that much of her experience in her department and at FIU are out of her control.

\section{Cuban-American Female Faculty}

Participant seven is an Afro-Cuban woman who identifies as Black. As mentioned earlier in this document, because of the study made one exception to the participant makeup, and that exception was to include members of the Hispanic/Afro-LatinX community who identify as Black. This was due to the racial heterogeneity of the Hispanic/LatinX community. She was born in Cuba and later migrated to the United States. The experiences she presented were substantially aligned with her experience or classification as Black despite her Cuban background even though she occupied a space that is predominantly Hispanic/LatinX. For context, when I asked how she identified, she made the following statement: 
I identify as Latina, ethnically, and as black...by race. Now, I know that in some theoretical sense... I could be considered a mixed race... if anybody else would have wanted me, I might have identified in some other way. But African-Americans in the United States have been the ones that have never, never questioned me, never tried to reject me. I love American Indians, but many of them are very [inaudible]. But that's a very long answer to a simple question, black. In Cuba, I will be called Afro-Cuban, Afro-Cubana.

In the above statement, her acknowledgment of rejection, and the African-American community having had been the only community to have never rejected her further qualified her inclusion in the study.

She has been with the institution for ten years, and much of what she expressed as part of her experience at FIU aligned with her overall experience in Miami. However, in presenting her experiences from this larger scale, she made it a point to state that Florida International University, from her experience, is the microcosm of Miami and that her experiences outside of the institution align with her experiences within the institution.

Much of her experience with regards to expectations and "extra" work she takes on are things that she typically places upon herself as a form of giving back and being a servant to her community. She acknowledges the additional workload; however, she does acknowledge the necessity for herself to do these tasks. The participant does attribute her cultural background (i.e., being Cuban) as a considerable contribution to her ability to effectively integrate herself within FIU; her Cuban background, although Black, allowed her to better fit within, and maneuver through the institution, especially as it relates to language. Spanish is her first language, although she is also familiar/fluent in French and English. Her ability to better fit, however, did not exclude her from cultural taxation as many of the experiences she shared with me fell under the classification of cultural 
taxation. She is nearing retirement and referred to our interview session as an exit interview in which she was able to be open and transparent about her time within the institution.

\section{Brazilian-American Female Faculty}

Participant eight is a multiracial young adult woman who is half Afro-Brazilian and half White. She has been with the institution for approximately seven years. She is a non-tenure track faculty who has a significant focus on service. She explained that she is in a weird place due to her racial ambiguity; she expressed that people usually cannot classify her racial or ethnic identity but that they know that she's different. When I inquired about her racial identity, she stated the following:

My biracial identity, sometimes they're not quite sure how to classify me. A lot of times, obviously, I'm not considered white, not necessarily considered black, not considered Hispanic. Kind of always in an "other" category. We've had this conversation. Sometimes when we talk about FIU as a Hispanic-serving institution, and then they say, "Well we don't have any Hispanic faculty." I'm like, well [pause] kinda, sorta [pause] but not really.

She was another participant that fell under the exception with regards to my sample selection. She attended the institution for undergraduate and graduate school. She has been active on several committees. She expressed that she often has a difficult time saying no. Much of her experience was presented through the intersectional lenses of being a woman as well as a woman of color.

Throughout the interview, her responses were concise but data-rich. It was almost as though she knew what cultural taxation was even though I did not brief her on the true intentions of the study due to the potential negative connotation of the term; The experiences she shared were engulfed with cultural taxation without her having had been 
introduced to the term. When introduced to the term "cultural taxation" I had asked if she was familiar with the phenomenon, mainly because of the way she responded to the questions, she was unaware even though her experience fits so well into the phenomenon. Her experiences provided clear evidence of the phenomenon. She was also very composed and not very expressive.

\section{Bahamian-American Female Administrator}

Participant nine is a Bahamian-American woman. She has been with the institution for three and a half years and serves in an administrative role. Her role within the university has exposed her to issues that relate to diversity within the institution. As a result, her lenses or understanding of the interview questions were heavily aligned with that. In addition, because of her role on campus, many of her responses related to the many instances she has been presented with by other FIU employees. While she did answer many of the questions regarding her own experience, she was also able to bring other people's experiences into the interview as well to provide additional data that I hadn't anticipated. Her interview was similar to interviewing multiple people at once because she was able to convey scenarios that she had been exposed to and tasked with solving, while also providing instances regarding her own personal experiences. When I inquired about her career progress, she also shared her exposure to access to employee progression across campus stating:

Because of what my office does, I get to see the progression of other people. Compared to [pause] particularly the black people, it's just not the same way [pause] hands down. That's obvious. It's not the same. So it does hinder it in the sense of - but it goes back to kind of like [pause] that lack of executives, that lack of [underrepresented] minorities in executive positions, you know. There's no one [advocating] for these people. There's no one standing up for them really trying to get more and more people in 
those higher roles. You know, the reason why we keep having people of other ethnic backgrounds get these roles these higher roles, because they have people, you know, advocating for them rooting for them getting these jobs, mentoring them, you know, like, whereas we don't have that, because we're in less and less of those roles. So, so I do think that has hindered the progression. I do.

I then proceeded to redirect her to discuss her, personal, career progress and her response was: "It's been limited."

She, too, appeared to carry a great deal of frustration with not only her experiences but also the experiences of the people that sought mediation through her office, providing several examples of cases she's encountered associated with employee relations. This participant is also no longer with the institution as she has acquired another position at another university.

European-American Female Administrator

Participant ten is a White, European woman. She has been present at FIU since she was 13-years-old but later attended to pursue and obtain a bachelor's and a master's degree. She presented a well thought out understanding of race, her racial classification, and what that could entail when working with students of color. She seemed to be very understanding; compassion and understanding were themes that came up quite often throughout the interview. She explained that she is the only European, non-Spanish speaking person in her office. She also explained that FIU is all she knows; she does not have experience in any other industry or institution. Language was a reoccurring theme throughout the interview, as well. She was one of a few individuals in her office that could not speak Spanish. She said that she has taken Spanish classes and has been able to 
"kind of pick up on some of it" but she has not been able to successfully acquire Spanish as a second language.

Overall, she expressed having difficulty maneuvering through the university because of her identity. When I asked her about any challenges she faces as a minority in her respective space, she stated: "I think, for me, it's: Are there any other white people around like...um, I don't know if we would do like a lunch and learn how to navigate a Hispanic serving institution." She also indicated that she does not feel included on campus. An example she provides was:

I think it's interesting, because we have Hispanic Heritage Month, we have Black History Month. And I understand that we don't really have anything specific to White people... White people have always had everything that they've wanted, so I feel like, like the minorities, such as AfricanAmericans, and Hispanics [pause] even though we are minority-serving institution [pause] — I think as a whole state or as a country understand why you want to hold on to that and make sure that those minorities are represented, but I don't feel like there's anything specific to white people on campus.

While she recognizes the significance of activities in recognition of minorities, she, in recognition of herself as a minority amongst an alternative dominant group contemplates the absence of the recognition of her cultural/racial group. Throughout the interview, she seemed to be very careful about how she presented her understanding of racial dynamics and how her identity may influence her interactions with minority faculty, administrators, and students in particular.

\section{Haitian-American Female Faculty}

Participant eleven is a Haitian-American woman and has been with the institution for twelve years. She currently serves in a non-tenured faculty role and is the only Black person in her department. She stated: 
I am the only African-American in my department out of 18 faculty, and no one in the staff.... And then as I'm attempting to bridge this administrative side of the house, as well as the academic side of the house, I tend to be, oftentimes, the only person in the room unless it's a really, really big room that has hundreds of people in it, and then I might be one of five.

She expressed that she often feels isolated amongst faculty, administrators and students. She mentioned that she had started teaching at FIU, Biscayne Bay campus "There was as a much larger African-American or black population there. When I came to this campus, I saw less and less as time went on."

This participant has multiple degrees to include two doctorate degrees. She expressed most of her experiences through a degree of empathy for her students of color. Over time, she has suppressed her cultural background while at work due to the responses she has gotten from students, much related to the negative stereotypes of Haitians.

Much of the interview revolved around the several committees she is on or has been asked to serve on in addition to often being tokenized. Many of her issues revolve around students not respecting her as they do her colleagues, and colleagues undermining her credentials and her expertise despite having more education than many in her department.

This interview had to have been one of the most discouraging and emotionally draining interviews of them all; the interview took an emotional toll on me because she is one of the participants I identified with the most, from personality to viewpoints, to the way she handles situations and the way she carries herself. Despite everything she is and has done, she still faces many unfortunate challenges within the academy; this interview 
felt like I was talking to a reflection of myself. Critical agency was a significant aspect of her experience.

\section{Jamaican-American Female Faculty}

Participant twelve is a Jamaican woman who has been faculty within the institution for thirty-three years. She has watched the institution grow and evolve. Throughout the interview, she shared her experience throughout FIU's transitional process. She was born in Jamaica and later came to the United States to attend school, where she started her experience as a "minority." She identifies as Jamaican. She has a more ethnic understanding of self than a racial understanding. She does not prefer to identify as Black, and she shares why in detail.

During the initial stages of the interview, the participant did not correlate her experiences with her ethnic or cultural identity, however as the interview progressed and began to delve into other questions, she then began to present her experiences more through an understanding of racial or cultural and ethnic differences. She reflected throughout the interview on some of her experiences outside of the institutional when she first came to the United States. She also reflected on some of her experiences as they related to situations during the Civil Rights Movement era; she made several parallels to those experiences and her experiences at FIU.

While on the surface it may have seemed as though her responses were disorganized and off topic, everything led back to a focal point when she explained that her experience within FIU was simply a continuation of the experiences she had been encountering over the years as a minority within the United States. I noticed that she was very culturally sensitive and aware of the intricacy of our differences as humans. She was 
very careful about how she described people regarding their racial, cultural, or ethnic identity. When asked if she found anything about her status as unrepresented to be taxing, she said no and then explained that she found it to be more "disappointing" than taxing almost as though it had become an internalized aspect of her life, thereby transforming her experiences from an added tax or burden, to a part of her being, hence having a more emotionally drawn understanding of the phenomenon because it is not just a "phenomenon" but a part of her because of who she is.

She is very strategic in how she works towards incorporating more diversity into her department. She also uses her position to expose the FIU community to what she refers to as the "other." She has worked on several committees and has even been a part of a committee specifically designed to ensure access and equity with the FIU community, which she expressed with regret has degraded into nonexistence over the years.

Iranian-American Female Faculty

Participant thirteen is a woman from Iran. Her experience was very removed from any relation to any form of cultural taxation. Due to her area of work, she felt her underrepresented status from more a gender standpoint. Additionally, she appeared to have initially understood the interview through a lens of discrimination, which was not ideally the focus, although discrimination subconsciously came up throughout a number of the interviews. As a result, in order to direct the interview towards its intentions, I had to clarify the intentions of the study with her again; from there, we were able to move forward. 
She was pretty brief with her responses, and this may be since she could not identify with what she may have thought I was "looking for." However, upon explaining the concept of cultural taxation after the interview, she immediately began to discuss instances that she has observed regarding others. Her voice and responses became much more extended and energetic. Her first response was: "That is totally [pause] I mean [pause]. That is not my personal experience [pause] but I have seen it in faculty. She began to give examples about a part-time, Black, male faculty member in her department. She even admitted to her acts of potentially taxing Black faculty when it came to recruiting members for events and search committees. It was almost as though she had an epiphany.

What my experience with this participant highlighted was the existence of the potential for members of the academy not to be fully aware of their actions- to recognize that their actions are in some way "off" but not fully processing the implications. She did not have much to say until I articulated what the phenomenon was and how it influenced the experiences of underrepresented members of the academy. She recognized her actions as individual entities but not how they may compound themselves and weigh in on underrepresented individuals.

\section{African-American Male Administrator}

Participant fourteen is a millennial African-American male that has been with FIU for three and a half years at the time of the interview as a program manager and interim assistant director. Much of what he expressed during his interview centered on being excluded and not having the autonomy to conduct activities that would allow him to serve as a critical agent or grow professionally. He felt as though he was being boxed into his 
role, type-casted. He spoke on feeling like people around him were trying to mold and place him into a role related to multicultural affairs. He understood his experience through the intersectionality of being in his position rank and not being a part of the majority group, which he expressed as being a "cloud[ed]," exclusive culture that does not allow him to integrate himself fully. He appeared to be frustrated, and a bit discouraged by his experiences. He came from an environment where he felt valued, which he did not necessarily feel in his current space. His responses were particularly interesting because he had previous experience working at a predominately White institution (PWI); that experience allowed him to provided parallels that were valuable to the study. His parallels supported claims that cultural taxation is not a predominatelyWhite-institution "thing," but perhaps a dominant-dominated "thing."

\section{Trinidadian-American Female Administrator}

Participant fifteen is a Black, Trinidadian-American woman in her early forties who has been with the institution for five years at the time of the interview. She did not necessarily see herself or her experience through a lens of race. Much of her experience was understood as a result of herself and her work ethic. She had not articulated many experiences related to cultural taxation. She did, however, expressed that she felt underrepresented in her department; her underrepresentation was felt based on being one of three administrators in her department that did not speak Spanish. She spoke of the presence of dominance and a sense of "familiarity" among the Hispanic members in her workspace. She also made mention of wanting to recruit and bring more Black students, specifically Black male students into the program she works with but that it was mostly out of her control. She also felt that not being Hispanic served as a disadvantage in her 
workspace, but overall, she has been able to bring herself up professionally within her department strategically.

Her understanding of herself and experiences were confirmatory with regard to having had come from a homogenous country. She also made mention of previously being employed at an organization that she considered toxic, so she often made references to that environment to justify that her experience as an underrepresented minority at FIU was not bad because it was better than her prior place of employment.

\section{FIU as a Microcosm of the United States (Parallels)}

"The academy is often imagined as an idyllic place, neutral and untarnished by the ugly inequalities that mar the "outside world"' (Stockdill \& Danico, 2012, p.1), however, throughout the study, participants made references to parallels between their experiences within FIU and their experiences as minorities within the United States. I include the section about understanding FIU as a microcosm of the United States to ground the participants' experiences and provides a greater understanding and perspective of the stories they shared throughout this dissertation.

FIU is an institution made up of predominately minorities (77\%), however, some of the numerically underrepresented members who participated in the study expressed that their experiences at FIU mirrored their experiences outside of FIU in predominantly White, non-Hispanic/LatinX environments; this was consistent among participants with a history in South Florida and those from other states, and countries.

Harbi (2016) states that "minorities widely share prejudice opinions towards other minority groups." When power and prejudice are coupled, there is the potential for 
racism to exist. Some participants' articulation of references regarding the FIU-US parallel include a Black, female faculty stating, "You don't get to forget your race in this country, at this institution, it's always there. No matter where you go, because there are not as many of us as we would like to see," which highlights how even though she occupies a space that is understood as divers by many of its occupants, she still shares similar sentiments with her experiences within the broader scope of the country. Three other participants reported similar feelings about the institution and how it is a "reflection" of society. A Black, male administrator expressed that "the same issues we deal with in the world we deal with on this campus," which further extends the significance of the study as many of the experiences are similar to experiences presented in studies conducted in predominately white spaces.

Feagin (2013) states:

Racial oppression makes the United States very distinctive, for it is the only major Western country that was explicitly founded on racial oppression. Today, as in the past, this oppression is not a minor addition to U.S. society's structure, but rather is systemic across all major institutions (p. 2).

The prior statement can further lend itself to understanding the link between the FIU-US parallel. In the subsequent section, I present how cultural taxation could be understood as a form of oppression; in this case, workplace oppression. The participants shared reflections could indicate that even though an environment consists of minorities predominately, when there is a dominant minority group among the population, that group may adopt behaviors of "traditionally" dominant, oppressive populations. Some of the participants' experiences in the workplace coincided with many of the components of Young's (2013) Five Faces of Oppression. 
Iris Young, in discussing the cultural imperialism promoted by and through the nation-state, defines oppression as "the disadvantage and injustice some people suffer not because a tyrannical power coerces them, but because of the everyday practices of a wellintentioned liberal society" (Young, 2014, p. 5). The nation-state, however, is not a singular entity. What we can call the nation-state is the summation of several social institutions, which include, but are not limited to, educational institutions. Young expresses that oppression is a "structural phenomena" (Young, 2014, p. 6), which is where my argument parts from hers to an extent. Young states that oppression is a structural system because it is "embedded in unquestioned norms, habits, and symbols, in the assumptions underlying institutional rules and the collective consequences of following those rules" (Young, 2014, p. 5). And so one must look to the ways in which individuals experience their homes, workplaces, and so on, in order to uncover these rules. My arguments about oppression, as it relates to the experiences of my participants, are based on what I understand as more covert structures. These covert structures are not written in stone or policy, but are a collection of unspoken, yet practiced rules and behaviors.

The experiences the participants shared during the interviews were expressed to have been practiced and implemented by the dominant group at the institution, an institution that plays a significant role in larger social forces through the knowledge it produces as well as its gatekeeping function for professions. The covertness of this kind of oppression I make an argument for makes this kind of oppression more challenging to spot and address. The covert nature of the situations expressed by the participants leans away from the more structured understanding that Young (2014) acknowledges. 
In relying on Iris Young's understanding, I am not comparing the experiences of underrepresented faculty and administrators to those of oppressed groups presented in her work. But Young's framework is useful for highlighting how the experiences in institutions of higher education get linked up with the larger oppressive forces to constitute cultural imperialism. There are parallels between what happens in the university and what happens in the nation-state. It is essential to understand oppression in all forms and in all institutions. In a sense, Florida International University is a society of its own, understood at a micro-level.I recognize that some of the participants' experiences with oppression in certain spaces on campus and the grander society (i.e., the United States as a whole) may not be mutually exclusive; that their experiences with oppressive acts can extend beyond the university space explored in the study. I also recognize that some participants may have the privilege of "escaping" an oppressive campus environment between spaces (i.e., work life and personal life). I, however, am only accounting for the experiences of the participants during their time present at, or in association with the FIU.

In the study, participants reported instances that can be understood as marginalization while reflecting on their day to day experiences on campus. A Black, male administrator stated the following: "A lot of times, for me personally, I feel like an outcast because I'm not Hispanic." He spoke of instances in which he felt marginalized because he did not identify with Hispanic culture. One of the biggest marginalizing factors for him was his inability to speak Spanish- also expressing that he has been corrected by others when he spoke in English to speak in Spanish. Another Black, female faculty expressed feeling marginalized by language, sharing instances in which she would 
enter the office and/ or meeting and: "and staff were speaking in Spanish and wouldn't acknowledge [her]."

Another participant expressed feeling as though he was not a part of any decision making within the institution.

So, I also come from a place where I like to be a part of what's happening. So that is very different for me to not be a part of that - And so, you're not a part of some of those conversations [pause] you feel kind of left out...So, I think for me, it is it's a double whammy. One, not being Cuban, or being of Hispanic or Caribbean island descent... And so, I'm asking myself; this is more recent, I think, like, what [pause] am I trying to force myself into like, being like this person and stressing myself out, or something like that [pause] that maybe I will probably never be included in. I mean, I think some of it is just not feeling included in what's happening - not being part of some of the conversations of the decisions. I think anybody wants to feel like somebody... Then I think sometimes when you look up and you don't see people that look like you as a part of those conversations like [pause] like it's disheartening. And so, I think sometimes the difficulty [pause] being a [pause] trying to find an identity within this culture. -Black, male administrator

The prior statements highlights how underrepresented members of the academy may feel, or actually be left out of decision-making on campus, in addition to an observed lack of underrepresented individuals in positions of power within the institution. The participant had actually previously worked at a Predominately White Institution, which further added to his frustration because even in that space, he did not feel as powerless as he did at FIU. The more puzzling part for him was that he was experiencing this among a minority dominant demographic.

Social marginalization also surfaced during interviews:

I don't feel integrated. I'm happy; I'm blessed, I move on. It's a job [pause] I'm grateful for what I have; I'm humble. But no I don't feel integrated. If you want me to be integrated in your community and to feel a part of the community, then dammit include me in the community... So if you want me to feel included, look at my culture, you look at my 
environment, and you bring those things to FIU, and then I will feel included. - Black, male administrator

This case presents a perceived case of social marginalization. The participant expressed, sentiments that he felt as though the institution mainly catered to the dominant group despite the presence of other cultural groups on campus. Another participant shared similar feelings, stating the following:

I really feel like there needs to be more work in terms of promoting diversity and promoting Black cultures. Sort of like integrate. Whether or not necessarily just Black stuff, just Hispanic stuff, but integrate the activities and make them more diverse for everybody, for all communities to be included... but that's kind of what I experience really, that there's still sort of like the segregation, rather.

-Black, female administrator

The participant expressed perceiving a degree of segregation and isolation on campus.

Further accounts regarding social and cultural marginalization were provided as a Black, male administrator:

I didn't have any expectations. I knew the demographics of FIU being Hispanic. What I didn't expect was [pause]. Well, I guess you could say I kind of expected more inclusion being a minority. Unfortunately, that's not the situation I see now. I don't know if it's something that's real conscious here in the environment, the workplace at FIU, but I have noticed that if you're not of Hispanic heritage or culture, background [pause] the culture itself in the workplace is [pause] maybe a little bit harder for someone who's of a different ethnic background because of the understanding of the language and the way things are done.... For example [pause]. When you're in the workplace, and you have a meeting such as a conference room meeting, and you speak [Spanish], you know what I'm saying, that shows that there's really not an awareness of other individuals who may not understand the language...that's in the room or the environment itself. I think it's just something with FIU, it's been based on the Hispanic community and the culture that they're in, but I think what they have forgotten also is that being that they are an international university, they have to be able to, I guess, gravitate and tend towards those who also may not be of the culture themselves. They say they're worlds ahead, but sometimes I question that... I will say being in an all Hispanic culture; you just don't feel that inclusiveness. For me, 
personally, I don't feel the inclusiveness. Even when it comes to interacting, very few people in the work office interact with me, and I think it's because I'm not of a Hispanic culture.

The participant had a clear understanding of his perception of the environment he occupies. The intentionality of the actions of the dominant are unclear. It could be that the behaviors of members of the dominant group are influenced by comfortability, but it could also be a way to assert dominance in the respective settings. We were unable to confirm nor deny either possibility. A White, female faculty member also shared reports of marginalization:

I don't feel totally integrated... because there's always groups and I would say it's an English thing, and sometimes Hispanic male thing. So not fully, no. And then there's physical barriers, so no, because of that.- Picnics, school picnics. There are things I physically cannot go to, so I can't be integrated because of that because of [my] physical disability.

The intersectionality of her being a disabled, White woman further magnified her experience with marginalization within the institution. She provided several accounts about how she felt marginalized linguistically, socially, and physically. If the series of behaviors expressed by the participants in the aforementioned accounts are understood as actions that could expel specific groups from useful participation in campus social- and or work-life, they can be argued as a form of marginalization. One Afro-Latina, female faculty spoke of being able to minimize her degree of marginalization due to the leverage being bilingual gave her as she states: "I speak Spanish as a first language; that was my first language. That is, that helps a lot with my colleagues. I get away with a lot just because I can speak to people in Spanish.”

Ahmed (2007) states that "Diversity in this world becomes then a happy sign, a sign that racism has been overcome" (p. 164). In this case, one would have to investigate 
what diversity means. How is it defined and perceived? Some of the participants in the study do not necessarily perceive the institution as being diverse. Statements about the diversity of the institution include:

To think that a university this big, with thousands of employees, with thousands of students, that a university that claims to be all about diversity, these are still the ideas and thoughts that like [pause] the faculty [pause]. -Black, female faculty

For context, the above statement is in regard to statements made by faculty in the department. The example given was about hiring other minorities within the department. The department was facing a potential hiring freeze, and a faculty member said to the above participant: "What does that mean, we have to start hiring incompetent people now?” This statement illustrates how some members of dominant groups may perceive Black; it may also explain the absence of Blacks in the department. Other comments about diversity within the institution include: "I think it'd be interesting to have some diversity training, but not from a Hispanic perspective. Maybe from like an outsider." The prior statement was made by a White, female administrator. The fact that she said "not from a Hispanic perspective" may indicate that there is a disparity between the ways that different racial, cultural, or ethnic groups conceptualize diversity within the institution. An additional comment regarding diversity from a Black, female faculty member included:

You get put on all the committees because they need diversity. And when there's not enough of you [pause] you become the one person who will become the diversity thing... When we lost the access and equity committee [pause] to a degree [pause] I think we lost it when we got bigger [pause] diversity was missing. 
When an environment is understood as diverse, however, certain members of the community express being over-utilized because there is a "need" for diversity, it presents what could be understood as a disconnect between actual diversity and perceived diversity. It also creates a situation that may invite discussions about how diversity is defined by different members of the institution. The participant also attributed the reduction of diversity to the loss of the university's access and equity committee, which could allude to the need for organizational forces to promote diversity.

The following statement is referenced in a subsequent section, however, I utilize it here to magnify and support my argument about perceptions of diversity. One of the participants, an Iranian, female faculty stated: "Universities require the diversity of the committee for search committees for the research faculty. So, we have like, one Black guy, and that guy has to be on every freaking committee. I was embarrassed." Her statement could create a space for dialogue about the comparison of the concept of diversity versus difference. I understand diversity as the presence of an array of several different classifications of individuals, and difference as the presence of an individual of a different classification.

The perception of diversity can influence the ways individuals make decisions regarding diversity within certain departments. A Black, male administrator said, "Are we making these diversity suggestions or decisions without having a diverse panel to be a part of that? So, it's kinda [pause] it has me in a place where I am unsure of the direction that we're going." This, too, could align itself with the different perceptions of diversity and how those perceptions could influence decision-making. If individuals perceive the environment as diverse, even if there is an insignificant number of individuals from other 
racial, ethnic, or cultural groups, there could be disconnects about the need for diversity

efforts.

Another participant made the following statement regarding his perception of

diversity within the institution:

Unfortunately, sometimes I say they contradict themselves, because when I look at their slogan, "We're worlds ahead," and then I look at their environment with the faculty and the workers here, it doesn't go hand in hand. They want to be diverse, but they don't show diversity. They want to show open-mindedness, but are they really trying to be open-minded? Are they tailoring towards a certain demographic, or do they really want to be worlds ahead and touch into every ethnicity group possible and really understand their backgrounds and cultures and the struggles they each have in this country?... On the student level, they're very diverse. Very diverse. We had a couple people from Russia, couple people from Germany, Haiti, Jamaica, Indian, Cubans. We even had one guy from Australia... and I think if FIU was to take that approach in their workplace, I think they would be a lot further than what they are now. For those who, I say, that are currently working at FIU, I would tell them to try to be more of a voice to encourage more diversity and to bring awareness to the workplace here. -Black, male administrator

While he does not perceive the institutions' workforce as diverse, he does perceive the student body as diverse. He also articulated what he felt was a presence of contradiction; that the institution prides itself on being diverse, however, he does not understand the space as such. A Black, female administrator shared that the institution "seemed diverse" when she first arrived but stated a confirmatory statement to my analysis of why the people may consider the institution to be more diverse than others may consider it:

It looks diverse, seems diverse, it's in our name [Florida] International [University]. But then when we look deeper, particularly as you get higher up, when you get to your tenure level faculty, when we get to your [pause] your administrators that are high [pause] any higher than assistant director, you kind of see that lack of lack of diversity there and sort of a, an ignorance to it as well, where people thinking that it's diverse, because they see themselves but not looking at it through the purview of other 
people... When you come here, you're going to think it's diverse if you always see people that look like you, especially if you're coming from a place where you've never, maybe you've never been in a [pause] you know, a place where everybody looks like you so you come here and it's great. And like, "Oh, we're diverse!" Like "Why are you having a diversity week? We're diverse; you know why you shoving this down our throats? Like, we're already diverse. You know, it's in our name." But you're not.

A Black, female faculty shared similar views regarding the university hierarchy, Stating:

"Not many African-American people are in a position of power, so to speak." In addition, the participant also expressed that she encounters members of the institution that question the need for diversity-related programming on campus. What that exemplifies is an unharmonious understanding of the environment; if one does not see a problem, they may be less likely to try to "fix" it.

One of the participants expressed that although he did acknowledge that the institution is diverse, there is still underrepresentation. He does so by presenting his perceived statistical understanding of the demographic makeup. The numbers are not factual, but are the way he sees the population at FIU:

I feel underrepresented because I feel like the diversity that is here at the university, is not diverse. I think it's $80 \%$ Spanish. $10 \%$ Whites, and maybe $2 \%$ Black. So, as far as my ethnicity is concerned, I don't believe we are [pause]. I don't believe the diversity is equal here [pause] I feel like my culture is not represented fairly. As far as diversity is concerned, at work [pause] if we're diversified here, it should be equal numbers of diversity here. Or we're not diversified... We use the term diversified loosely... But, besides the diversity issues, it's a great school. I like it. I'm proud of FIU. -Black, male administrator

A Black, female faculty stated: "I think even though it's not as diverse as it could be, there is diversity in a way in terms of where people are from, as opposed to race." She spoke about her encounters with people from a variety of Hispanic and LatinX 
backgrounds- thereby presenting the existence of diversity, but within a specific demographic, which could, too, contribute to the varied perceptions of diversity. From an "outsiders" perspective, i.e., someone who is not a member of the Hispanic or LatinX community, one's understanding of the diversity on campus may be skewed and vice versa.

Only one participant made a confirmatory mention of the institutions diversity, in which he states: "I love the diversity. I love the different languages I hear all the time." That statement was made by a White, male faculty member who had come to Miami from a much more homogenous area. So, this again begs for the definition of diversity. Is diversity being defined as the presence of people from a variety of racial, ethnic, gender, religious, linguistic, socioeconomic groups or as the presence of someone/something different from oneself, or the presence of predominately the/ an "other," i.e. a nonpredominately White space? The participants' take on diversity on campus also begs for discussion regarding diversity and inclusion, as do some of the prior accounts.

"Powerlessness" (Young, 2013, p. 2) as a form of oppression can also be taken into account as a part of the experiences of some of the underrepresented faculty and administrators in the study. Young (2013) explains that the powerless do not participate in any decision-making processes. Some of the feedback from the participants include:

So, I think our institution now is going through like a transition. And so, the transition that I see us going through is that we're losing some senior administrative staff [of color], some mid-level and senior. And so, during this transition, I've seen some people come in... but there's no senior leadership, at least on our side [pause] persons of color...And so, as time goes on, I feel like this is, somewhat concerning, to not feel like we have leadership put in place to bring [their] view to whatever decisions are being made. -Black, male administrator 
What he expressed was a sense of institutional powerlessness in which he observed a lack of representation in leadership positions that can serve as a voice for members of underrepresented groups, in this case, Blacks. During the interview I inquired about his use of language in terms of the phrase "people of color" and he clarified that he was referring to Blacks, even though he understood Hispanics and members of the LatinX community to be people of color as well, although, from his experience, many of the Hispanic/ LatinX people he has encountered do not identify as people of color, but rather, white. An Afro-Latina, female faculty member shared similar sentiments stating that: "I think sometimes that [pause] unless you really put yourself out there, then you don't find out about these things until after the fact, and you don't have a say in how things go." A Black, male administrators attributed the lack of Blacks in positions of power within the institution to the concept that "Some feel that we [Blacks] can't be in a position of power. I don't know if that's permeated through management as much as it used to be, but those beliefs still exist, unfortunately." This was a common understanding among the participants. One attributed the lack of Blacks in positions of power to the following:

I mean, because this, it is a [pause] it's [pause] is a heavy cloud of Hispanic. And I don't want to sound too negative when I say that, but like [pause] it is a very close-knit culture. And, and I think it reflects in some of the hiring and some of the people who sit in some of these other conversations. -Black, male administrator

The use of the term "cloud" could be indicative of a perceived negative presence. "Heavy," indicative of a significant amount. The participant correlated his understanding of the dominant groups' close-knit relationship to some of the hiring practices that he observed during his time at the institution. To perceive a hiring practice as one that leans towards hiring a certain population speaks to how the environment could create a space 
that culturally taxes people from certain demographics- there may not appear to be efforts to diversify the population- hence, reproducing environments that increase the extents that underrepresented members of the academy are culturally taxed. A White, male faculty member stated: "There's only so much a person can control... You just let it go," which too can be understood as a sense of powerlessness- the inability to change a situation and make it more desirable for oneself.

Continuing with the critique of powerlessness is Freire's (2005) addresses a culture of silence, which could also be tied to the previous statement. Such a form of powerless has the potential to birth a culture in which it is forbidden to voice the injustices being committed. Where this applies to the study is in two areas. The first area is a trend that was documented following some of the interviews. Whenever some of the participants mentioned the term "Hispanic" or "Cuban," they would lower their voice to almost a whisper. There appeared to be a degree of either discomfort, fear, or nervousness when using the terms. Another theme that arose was a reluctance to speak up about things that bothered them in the workplace. The following text presents samples of some of the statements made by the participants. A White, female administrator stated: I feel like they only treat me that way because I don't speak up. But then my concern is that at the time when I do speak up, I'd be considered offensive.

The above statement regarding silence is grounded in a fear of being offensive, which is also grounded in the participants" racial identity among a "minority" group, even though they are dominant in her respective space. Her silence is linked to a degree of sensitivity, and not wanting to confirm certain stereotypes that she acknowledged may be tied to her racial identity. As a result, she finds herself in a number of uncomfortable 
situations in which she feels as though she is being treated unjustly. In other cases, participants expressed that although they felt silenced as it related to being able to vocally express dissatisfaction to their peers directly, they, in turn, utilize other outlets to address their concerns. A Black, male administrator articulated that "Sometimes [I] write miscellaneous letters to different people about issues. ...If I go complain, there is backlash, there could be backlash to me." So while there is a degree of "vocal" silence, he finds a voice within strategy and anonymity.

Another Black, male administrator highlighted a perceived absence of a voice on campus when he says: "I think a lot of individuals do what I do, is we kind of just sit back and we don't say [anything] at all" and when he presented his advice to underrepresented member of the institution he said to: "Be more of a voice to encourage more diversity and to bring awareness to the workplace here."

A White, female faculty member expressed the following: "So I feel like I've not been in a position where I feel secure in my working environment [pause] and so not fully integrated, and not wanting to make any waves for that reason." She attributed her silence to the fact that she is not a part of the "inner circle," which, in her spatial composition, is mainly Hispanic and male. As a result, she expressed that she doesn't speak up on certain issues in her department- ultimately silencing herself. Another AfroLatina, faculty member also referenced the avoidance of making "waves" in her department.

Ultimately, participants expressed external and internal pressures that influenced their silence on campus; some were related to conflict avoidance, some were related to avoiding backlash, while some were related to feeling like they did not have the power to 
voice themselves. Some were aware of the implications of their silence and advised that other underrepresented members of the institution use their voices as a way to inflict change.

In continuing with Young's (2013) critique of oppression, some participants expressed instances that the dominant groups' behaviors reflected beliefs that their culture and or presence is invisible or unworthy- these instances are understood through the lenses of cultural imperialism. I open this segment with a stamen made by an Iranian, female faculty member:

I think not being as a part of the majority-minority, which is Hispanic... I feel like, you know, I have a harder time to try to get it. It's not as like, it's not like the other institutions, [FIU] has its own culture. And you have to be [pause] I mean, dealing with other people I feel like okay, you know [pause] I'm not [pause] here.

In this case, the participant expressed a sense of invisibility because she does not identify with the dominant group. An important aspect of cultural imperialism is that members of dominate groups deem their meanings and culture superior to others, rendering others as invisible or insignificant. A Black, female administrator stated:

I think that [pause] I think it's just the Hispanic culture, I think they're just very closed-minded to how they come across with their strong culture. And you know, it's not comfortable to be in a room and everyone's speaking English and [pause] and all of a sudden, you speak Spanish. And you know, there are people in there who do not speak Spanish, you know, to me, that's just rude.

The participant presented a scenario in which the dominant group intentionally or unintentionally renders others invisible by ignoring their presence in certain spaces and isolating them from certain conversations which, according to some of the participants, exceed the social realm and expand into work-relations. A Black, male administrator 
said: "Don't let this university try to constrict you or change your culture, you know."

This followed instances in which he spoke of what he perceived as the prevalence of

Hispanic culture and a lack of acknowledgment or appreciation for others- an

environment that called for conformity in place of tolerance. A White, female

administrator spoke of instances in which she would find herself in a situation that could

be understood as cultural imperialism through linguistic practices. She stated:

I have an administrator in admissions that every time I see her, she'll talk to me in Spanish, and like a whole long conversation. And I've reminded her many times that I don't speak Spanish. But she'll just continue on in Spanish. So it's like [pause] okay, do I [pause] do I come off as being rude, saying, "Don't speak to me in Spanish?" So how to navigate that conversation can be challenging.

The participant could not confirm nor deny the intentionality of the woman's actions;

however, she said that has made the woman aware of her inability to speak or understand Spanish even though her actions persisted, ultimately ignoring the participants' linguistic abilities (or inabilities). A similar account was presented by a Black, male administrator:

Here's an example. I go to the [campus restaurant] [pause] I get my bag, I'm ready to go [pause] I say, 'let me get a spoon.' The Spanish lady tells me 'you mean cuchara?' - Okay, wait a second? I'm not here to learn Spanish right now. I'm just here to get a spoon. And if I ask you for a spoon, why do you think to take it upon yourself to now teach me Spanish? Do you want me to teach you English? If I ask you ma'am, can I have a spoon and you say "no, cuchara" am I supposed to now say, "no, spoon?" So that's the stuff I deal with... I feel like anywhere I go on FIU, I shouldn't be taught Spanish. Nobody should ever correct me. I'm a grown man. As a grown African-American man, I don't know no [traditional, African] language. It was taken away from me. So you took away my African language. You allow other cultures to still speak their languages. Fine, speak your languages. But now you're going to make me learn your language too while I'm at work? Those are some of my experiences. 
His account included an FIU affiliate employee correcting his use of English at an establishment. This could be argued as a learning experience and an opportunity to take advantage of one's surroundings to practice and learn another language, but the lack of choice or control over one's decision to participate in the acquisition of another language is the participants' matter of concern. In a statement presented in a future segment, a White, female faculty states:

Well, I often feel like, different, and the differences are clear when somebody speaks Spanish, and it feels like all of a sudden, there's a club that I'm not part of... When there are just meetings, and mostly when people speak Spanish, I feel like it's just, to me, it says, "You're not part of this."... It definitely feels like without a doubt, I'm an outsider for sure. Like, I'm not an old friend because my [pause] no matter what I do, I'm still an outsider, not an insider.

I would argue an instance like the previous one as cultural imperialism if the actions are committed intentionally by bilingual or multilingual individuals- which, in her case, they are as she mentioned throughout the interview that her colleagues would often switch to Spanish during a conversation being held in English, during work and non-work related conversations rendering her and in some cases, other colleagues invisible. In another language-related instance, a participant shared her experience having to mediate a case on campus that could be understood as a form of cultural imperialism:

[Example] An employee calling another employee loud and ghetto because they are speaking loudly in their native language? But you have your other coworkers in the same exact office who speak Spanish loudly. Why isn't that ghetto? But you want to report this employee, you know, formally report them for being loud and being ghetto... [And in another instance] Because they have food that smells [pause]. If they had [pause] if they had croquettes would you have said that? Again, if they were speaking their Spanish loudly, which they do, do you ever report them? Black, female administrator 
The acknowledgment of a colleague as ghetto because they speak/spoke loudly in a language that differs from one's own native, or preferred language could be a way of deeming the other language as less than or unworthy- it also depicts the absence of tolerance for "others." The employee reporting their colleague for speaking loudly despite other colleagues speaking loudly in Spanish within the same office alludes to the potential presence of bias.

A Black, male administrator articulated: "I think that culture has a heavy presence in the workplace." This statement indicates that there is a perceived cultural dominance, which other participants also articulated throughout the study. This notion may aid in understanding how one may experience cultural imperialism as a form of oppression- and cultural taxation being understood as a form of oppression.

Said (2012) explains that "The power to narrate or to block other narratives from forming and emerging, is important to cultural imperialism. There is power in numbers, and the perceived daily imposition of power in the dynamics of everyday work-life within the institution has was presented as a concern by participants in the study. However, despite what the participants presented, an understanding of their experiences could be explored through the argument that the institution is classified as a Hispanicserving institution; therefore, the environment, demographics, "society," and/or culture would reflect that classification.

Again, this section is imperative to aid in the understanding of the participants' experiences and the relevance of exploring cultural taxation in an institution like FIU, which has a demographic unlike institutions where cultural taxation has traditionally been explored (i.e., PWIs). Understanding Florida International University as a microcosm of 
the United States assists in rationalizing why the underrepresented faculty and administrators in the study, who work at an MSI, might have similar experiences as underrepresented faculty and administrators at a PWI.

\section{Chapter Summary}

This chapter serves to ground the participants' experiences as presented in the subsequent chapters. The spatial context aids in reducing potential misconceptions that some may have when conceptualizing the environment at FIU or other non-PWIs. The data provided by the participants about their understanding of the spaces they occupy lends itself to the importance of the study. Given that the spaces explored in prior studies were predominately White, it could be assumed that there was no presumed need to investigate the phenomenon in a multicultural environment, however, the participants' feedback demonstrates otherwise. Although they occupy a majority-minority space, many of the experiences they shared mirrored those of underrepresented members of the academy in predominately White institutions.

Providing descriptions of the participants, to the degree of maintaining anonymity, was done to give the reader an understanding of each participant. The participants are presented vaguely throughout their profiles and the subsequent chapters. I intentionally took that approach to distance the participants' identities as much as possible to protect them given the nature of some of the topics covered and the fact that most of them are still currently employed by the institution, thereby privileging the data over its source. While this may not be ideal in qualitative research, I did not feel comfortable potentially putting any of them at risk of exposure and/ or potential backlash. I made this decision to muddle the data in a way that would reduce the readers ability to 
potentially construct an identity by following statements made by particular participant as they read this dissertation; for that reason I refrained from the use of pseudonyms as that could serve as a source to link to one's identity. I made this decision based on the reality that readers of this document are not limited to those outside of the institution, but can include those who work here as well that may be able to connect the dots of certain participants, based on their response, hence sacrificing anonymity. I present the participants by race, gender and position type (e.g. faculty or administrator) since there are, for the most part, multiple people that fall under either category.

I would have felt more comfortable using pseudonyms or some other less clinical approach had the institutions' name not been disclosed; if there was that kind of disconnect I would have been more open to more descriptive alternatives. While there is this disconnect, my hope is that the reader can privilege the data provided by the participants as an illustration of their experiences over the association of each individual participant to the data.

The participants reflected on their time at the institution and constructed responses about their experiences and their perceptions of their experiences. The participants were all classified as underrepresented in accordance to FIU's demographics, however not all of the participants were minorities as classified by the United States Census Bureau. 


\section{CHAPTER V}

\section{IMPOSITIONS OF CULTURAL TAXATION}

This chapter addresses how the participants' experiences relate to cultural taxation and how the phenomenon can take multiple forms. In understanding how their experiences entail cultural taxation, I use Amado Padilla's explanation of cultural taxation to introduce an overarching essence of the pressures and obligations minority faculty and administrators face in environments where dominant groups exist.

During the study I came across, what I argue as, understandings of cultural taxation which were not identified in Padilla's original work - this allowed me to take multiple understandings of cultural taxation into account, which are argued as such due to the time and energy they require and consume. Time and energy are vital in understanding the totality of some of the arguments made in the study, understanding that the time and energy involved in the activities presented are connected to ones' cultural, ethnic, and or racial difference in their respective environments. The participants generally expressed experiencing some form of cultural taxation or another. All but one of the participants expressed feeling underrepresented; the anomaly was a White, male faculty member. He did not identify with some of the topics that were addressed, although he made many subconscious statements about his experience that were directly related to cultural taxation.

While some forms of cultural taxation are more prominent than others in the study, I argue that one's experience with the phenomenon does not have to encompass every, or even multiple components in order to be considered culturally taxed. Whether one's experience relates to one form, or every form, the additional time and energy 
required of them is relevant to the understanding of cultural taxation. Again, none of the participants were informed of the study's intention to explore their experiences with cultural taxation prior to the study. The information/experiences shared were rooted in their own experiences outside of their understanding of the phenomenon.

In speaking with the participants, I was able to understand cultural taxation as having three motivational forces: external, internal, and social. While social impositions can also be considered external, its influence is more conceptually connected to the collective environment versus a phenomenon that is directly imposed by the administration or a member of the dominant group. Florida International University, as the environment for the study, provided a space that was shown to elicit culturally taxing activities from the underrepresented participants despite the demographics consisting of, predominately, minorities.

The chapter will also address the concept of tokenism, understood as an external imposition, because it surfaced explicitly among some of the participants and can be understood as a component of cultural taxation if it is conceptualized as a "contribution" levied on one and paid by their presence in an environment. The chapter will conclude by revisiting the published impacts of cultural taxation while introducing newly emerged impacts and understandings of the phenomenon.

I am warranting that the existence of underrepresentation, coupled with the existence of overrepresentation and cultural dominance, creates a space that could make some of its occupants vulnerable to cultural taxation. In addition, I am also warranting that cultural taxation can be understood as a series of entities; the ways I present its understanding are as: an obligation, a component or act of critical agency, a form of 
tokenism, and a form of oppression. The understanding of the phenomenon relies on the impositions of the cultural taxation along with the intentionality of the impositions. I would also like to reiterate how cultural taxation was initially defined for the studybecause of the evolution and broad application of the term, cultural taxation was understood as anything done, or expected of one outside of their job duties because of their cultural, racial, or ethnic differences. However, again, following the data collection and analysis, the final definition that I have come to use to define the phenomenon and its multidimensionality is "The additional physical and/ or mental demands imposed on underrepresented members of the academy irrespective of intentionality or origination."

I present the participants' shared experiences thematically in accordance with their impositions: External, internal, and/ or social, and sub-thematically in accordance with how the participants experience cultural taxation through expectations, service, tokenism, obligations, coping, and integration. Following, I will present the kinds of $\operatorname{tax}$ (or cost) resulting from their experiences, i.e., a tax on one's time, emotions, psyche, and finances. I include both emotional and psychological tax as separate entities by understanding emotion as a temporary part of one's experience; an entity that occurs and leaves, and psyche as something that is long-term and essentially becomes a part of one's life; it become a part of one's mental profile.

I organized the data in accordance with impositions to highlight and differentiate the multiple forces of cultural taxation underrepresented faculty and administrators can face in the academy. I take this approach because prior literature seems to understand cultural taxation as a unidimensional force, mainly originating from pressures directly imposed on individuals by the institution and members of dominant groups. I want to 
highlight how cultural taxation can originate from external pressures, internal pressures, and social pressures. This categorization creates an opportunity to lean away from a more concrete, inflexible understanding of cultural taxation; this allowed me to be more flexible in my conceptualization of the phenomenon, whereas its original understanding could be understood as a more stagnant and less applicable to circumstances that are even more "invisible" than they are known to be. Instances of cultural taxation that are initiated from within by underrepresented members of the academy and instances that are initiated by social climates are much less tangible than being asked, called, or directed to complete particular tasks. This categorization allows for future investigation of the complexity of cultural taxation.

Amado Padilla (1994) provided discourse on "the imposition of taxation because of cultural background." What I aim to do in this section is expound on the understanding of how different forms of cultural taxation are imposed. Following the data analysis, I interpreted three ways the phenomenon can be imposed on underrepresented faculty and administrators: externally, internally, and socially. Externally imposed forms of cultural taxation involve instances in which one is directly called upon, asked, or directed to complete certain objectives. Internally imposed forms of cultural taxation typically take place indirectly, as a result of another action, instance or situation that may or may not be related to cultural taxation; this form of cultural taxation is imposed by oneself; it is also linked to moral obligations. Socially imposed forms of cultural taxation involve acts that are in response to the social climate and/ or social structure typically set by dominant groups. 
This understanding presents that cultural taxation does not have to be a result of direct impositions, which could be loosely implied by previous studies, as Padilla (1994) argues cultural taxation as "situations that are imposed... by the administration." His discourse would fall under, what I argue as external impositions- the administration being the external force. The following diagram presents a visual of the impositions of cultural taxation.

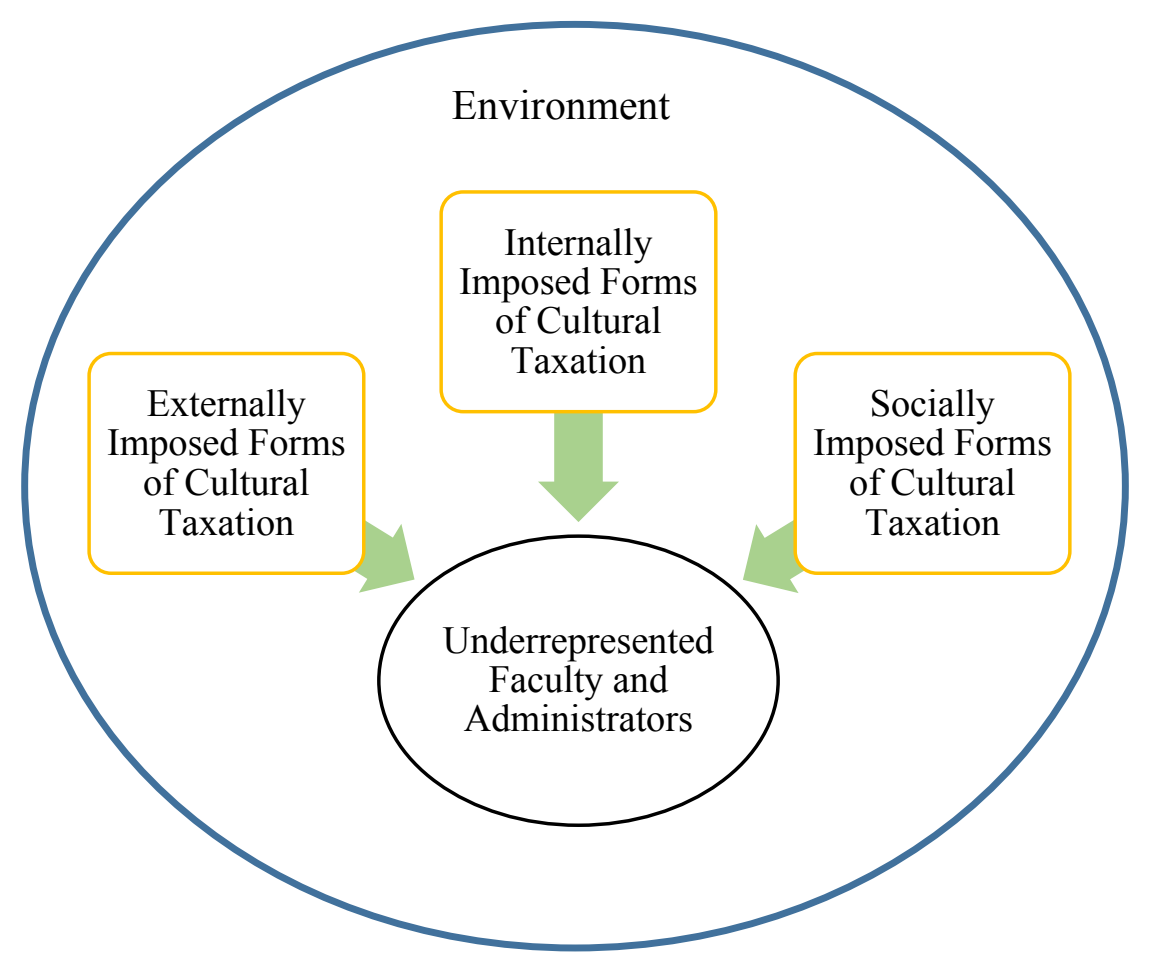




\section{External Impositions}

External impositions are experiences regarding cultural taxation that are initiated by the institution, colleagues, and/ or students. Much of the participants' experiences relating to external impositions of cultural taxation revolved around service and expectations. The kinds of service in the study have been understood as forms of general service or race-related service (Baez, 2000) done in order to serve the needs of the institution or underrepresented incumbents. In some cases, there can be a conflation of the two types of service. I apply their understanding, as presented by Baez (2000), to the study. General service entails "community, institutional, and professional activities that all faculty members are expected to perform [with] little direct connection to race, diversity, or social justice" (p. 374). Race-related service entails "any community, institutional, or professional activity perceived by the faculty members [or administrators] as benefitting their racial or ethnic communities" (p. 374). In the conflation of the two, some of the underrepresented members of the academy in the study expressed being called upon to participate in certain aspects of general service more often than others to satisfy the institution's diversity needs which can be understood as the institution perceiving the utilization of underrepresented members as benefiting the institution.

It can be argued that some service-related forms of cultural taxation can be induced by expectations whereas underrepresented faculty and administrators may be expected to serve the institution in specific ways, but for the sake of organization, I present service and expectations as separate entities; In addition, expectations do not only revolve around participating in race-related service but also in relation to presupposed 
aptitude and behaviors - the time and energy exerted in an effort to combat presuppositions justify the claim that these kinds of expectation induce cultural taxation.

In addition, I also present tokenism as an external imposition of cultural taxation. Throughout the study, this concept surfaced as a part of some of the participants' experiences. I recognize tokenism as an act that is orchestrated by an institution to give the illusion of diversity. The institution functions as the external force imposing this role on its underrepresented faculty and administrators.

\section{Expectations}

In the study, expectations are argued as external impositions as they are tied to the thoughts and beliefs of others that transcend over to the individuals they are housed about. Ten of the participants conveyed having had experienced unequal/irregular expectations within the institution. Some of their experiences aligned with irregular expectations as they relate to participating in diversity-related matters, while others were related to members of the majority group underestimating their abilities because of their race. One of the Afro-Latina, female faculty explicitly articulated that she feels and recognizes the expectation from her peers to participate in race-related service, stating with a degree of weariness in her voice that:

I am expected to, to be in the stereotypical things [pause] I'm expected to do all those things... Anything about taking students to the community, to do real community organizing in communities of color. I have to do it, because in part [pause] because it's expected of me... if there is a need to address issues of disparities, issues of equity, I embrace the expectation. But the expectation will rise embrace it or not, the expectation is there.

Essentially, she recognized that the expectation to participate in race-related service is tied to her being and presence despite her response to it, making this kind of cultural 
taxation, to a degree, inescapable. It can also make it bias being that there are other faculty member in her department that can participate in this kind of service, but because they are not "marked" by their racial identity, they can avoid these expectations.

One other example of race-related service presented by a Black male administrator was the notion of being expected to serve as an advocate for students of color. He felt as though the institution has an expectation and/or want for underrepresented members of the academy “To be that person who's like, saving these Black kids...Or these kids of color [pause] students of color.” The statement assumes that a specific demographic of students warrant "saving" or specialized assistance from the institution; however, instead of the institution generating the efforts, the efforts are expected to be generated by certain members of the academy because of their racial or ethnic classification/difference; thereby potentially creating an environment that might allocate disproportionate responsibilities to underrepresented members of the academy. This disproportion has been noted to contribute to burnout and reduced job satisfaction (Mahoney et al., 2008; Hirshfield \& Joseph, 2011; Pololi et al., 2010; Hassouneh, et. al., 2014; Mahoney, et al., 2008; Kolade, 2016; Trower, 2003; Blackburn \& Lawrence, 1995).

Another Afro-Latina, faculty member, expressed the expectation to be the face of diversity for the department, "Especially on committees where they're looking for diversity." Instances like this presuppose a high demand for diversity accompanied by a low supply of diversity in the workplace, which creates situations in which she is overutilized to compensate for the shortage of "others." In addition, the concept of "looking for diversity" highlights an absence of diversity. One's racial or ethnic difference, from 
what I gathered, assumes "diversity" within environments like the one she occupies no matter how minimal and lends itself to the idea/expectation that the underrepresented member(s) will act as the representative of diversity.

Preconceived racial expectations (typically lower) were also a perceived part of some of the participants' experiences. The time and energy exerted to deal with and combat these expectations qualify them as ways that underrepresented members of the academy can experience cultural taxation. These types of expectations can be tied to stereotypes and lack of exposure to "others." Six of the participants expressed cases in which either they felt or observed members of the dominant group make assumptions about their abilities.

Two Black female participants (one faculty and one administrator) made mention of instances in which students and or other FIU employees assumed that they occupied lower-level roles on campus than they did; one sharing the following instance:

I remember when I first started here, I would go to receptions [pause] and if I didn't put my name card on, you know, someone would ask me to get them a drink because they would think that I might have been, you know, part of the serving crew.

In the above case, Blackness and, potentially, the intersectionality of being a woman was abstracted with servitude, which speaks to implicit biases dominant groups may house.

One Black male administrator made the following comment regarding his Blackness:

I hate to put it like this, but I think me being an African-American male, there's a level of standards that are already set. It's something that you can't touch and it's something you can't see, but it's more something you can feel. 
These expectations are implicit, but they still make an impact on individuals on the receiving end. Something I feel is worth noting is that the notion of lower expectations surfaced among the Black participants only.

Their experiences with lower expectations were typically stated with anguish, frustration, yet acceptance. One Black female faculty expressed that she had "learned how to deal with the fact that the color of [her] skin meant that [pause] that [pause] [she] may not have been seen as knowing as much as [she] should.” The acceptance factor can be understood as normalization of these expectations and potentially create situations in which she and/ or underrepresented individuals anticipate these expectations and, in turn, feel the need to prove their value and ability (Campbell, 2015; Pololi, 2010). This form of cultural taxation is closely tied to differential legitimacy, which also surfaced through the interviews.

Nine participants admitted to feeling the need to prove themselves beyond what was stereotypically expected of them; ultimately, creating a situation in which they felt as though they had to work harder than their dominant counterparts to earn respect and/or acknowledgment in the workplace. One of the Black, female administrators mentioned that "The stereotypes that Blacks have in this country [are] burdensome, unfortunately... I feel that it's important for me to conduct myself a certain way. That way, I don't have the stereotype." Participants expressed similar sentiments expressing that their actions are "interpreted for the entire race," and that they feel like they "represent the whole."

Some of the other assumptions tied to the participants' experiences was the notion of their presence in their respective academic spaces being attributed to some sort of "affirmative action gift" as articulated by one of the Afro-Latina, female faculty: 
I don't necessarily crave equality. I just [pause] I do want it recognized that every single thing that I get, is not because I have a lot of melanin. Some of the stuff that I get is because I don't quit. I work all the time. Very hard... I don't mind the work. I again - the only thing that bothers me is the assumption that people who work much less than I do have; the assumption that anything that I get is an affirmative action gift.

Assumptions tied to affirmative action, especial conceptualized as a gift, presupposes that the individual did not have to work hard to get to their current position; it also presupposes that the individual under question may not necessarily qualify for the position they occupy but were instead given the position to satisfy some quota. In an effort to combat the stigmas tied to their minority status, some of the participants admitted working, "All the time. Very hard," having to "Stand out," going "Above and beyond to be a good employee, so you don't mess it up for everyone else" or "prove that [they're] worthy. To prove that [they're] equal.” The idea of not messing it up for everyone else also assumes a sense of "luck" or privilege; that their presence in their respective academic space is actually a gift being that there are so few in their areas, and that disparity places them in a position in which they are a representative of their minority group which, in some cases, creates an added pressure to excel.

A Black, female faculty member also admitted to passing these expectations and survival tactics down to her Black students by being "Stricter with the African-American kids, the Black kids generally." She stated:

My way of handling [lower expectations] is trying to get [Black students] to excel because that's how I got through. Yeah. You have to stand out. And so I do that a lot and maybe that's why I'm a little bit like more strict [pause] Stricter with the African-American kids, the Black kids generally. I'm maternal but I'm [pause] also my bars [are] high... [Advising them to] 'Cross your T's, dot your I's...' We are one group of people that judged [pause] we are one group of people that are judged based on the action of one person... It's burdensome but we're in this. - Black, female faculty 
Her efforts to prepare her Black students for what they may face in the future could be understood as a lack of hope for change; that she expects that her black students' experiences will mirror hers, so she works to equip them with the necessary tools to prevail in their professional lives.

Differential legitimacy even surfaced among some faculty despite their professional experience and credentials as one professor expressed:

You always have...you have to be on your best behavior all the time. Because any action you take is going to be interpreted for the entire race. To your tone, your dress, your hairstyle, your attitude, your [pause] the way that you write, the way you communicate, the way you carry yourself, everything... I have to be so careful with what I'm saying [ pause] and make sure that it all comes out perfectly in front of this particular group. And although they know that I have more of a writing background, and now a heavy teaching background and all this stuff in education, even then, I'm reluctant to speak unless I've written out literally what I'm going to say as if it's a script. -Black, female faculty

The above statement depicts a degree of self-policing and self-doubt as a result of the circumstances created by her environment; it also speaks to the performativity of work; however, in a case like this, there is no intermission; the individual is continuously performing.

Similarly, a Black female administrator articulated the following:

Yeah, I guess you could say that I feel like I have a responsibility to be [pause] I mean, everyone should feel like they have a responsibility to be good, but um, because you know, you are a Black female in a culture that does not have a lot of Black females in certain roles, you do feel [like you] kind of represent the whole, you know? And not, not be too loud, not be too disagreeable. You know, things like that you do feel a responsibility to just kind of go above and beyond to be a good employee, so you don't mess it up for everyone else... I mean positive is it makes you strive to really be the best, and you understand that when you do good, it's not just about you, you know? If you do something wrong [pause] when you do something good, you know, it's, um, it's good for the whole. But the bad 
side to that [pause] is when you do something wrong, if you make a mistake, it kind of [pause] it reflects on the whole as well, you know...I guess, it just goes back to that feeling of, you know, needing to really do your best so that it doesn't reflect negatively on other Black females.

She articulates a sense of responsibility to carry herself in a certain way, even though she recognizes that everyone should strive to be their best. In her case, however, it is almost as though she feels like she carries the weight of other Black women's' futures. Her performance is linked directly to the perceptions of other black women which lends its self to the lack of presumed individuality because of one's racial identity. This entails an added pressure, both physical and mental, that influences her daily actions.

When one of the Afro-Latina, female faculty was questioned about differential legitimacy, she expressed that she did feel as though she had to work harder to prove herself because she was, what she referred to as "an oddball in academia" which also speaks to the disproportion in racial and gender representation.

The statements made by both faculty and administrators align with Hirshfield and Joseph's (2011) and Pololi et al.'s (2010) research on how the perceptions of dominant groups impact members of underrepresented groups. In some cases, the participants felt as though they did not belong in their respective departments, in some cases they experienced distrust from their colleagues with regard to their qualifications, and in some cases, they did not feel valued which resulted in them feeling the need to work harder than their majority counterparts in an effort to gain their trust and respect. Differential legitimacy could qualify as an external and or internal imposition depending on if it is assumed by members of the dominant group, or if it is a result of prior experiences which 
could lead the underrepresented faculty or administrator to assume that their counterparts house such beliefs, even if they don't have those biases.

Other ways underrepresented members of the academy experience external impositions of cultural taxation is by being called upon to serve as the "expert" on diversity matters. As it relates to being called upon to serve as diversity experts, four participants expressed having had encountered this form of cultural taxation at some point or another. One of the Black, female faculty member shared that she had somehow become "The diversity advocate for the department" and questioned the optics of her acting in that role while being the only black person in her work area. The role of diversity advocate was tied to her racial difference, which is home to the core assumption that diversity or race-work is the responsibility of members of minority groups. She also stated that "The problem with being Black, when you're talking about race, and ethnicity, is that they expect you to be a subject matter expert, because you're Black, but they also say you cannot possibly be objective, because you're Black." This instance describes a double-edged sword expectation in which a minority may be expected to be a subject matter expert, while simultaneously having their expertise questioned because of their race, potentially creating a situation in which specific race-work may be done in vain, or the individuals may be participating in what Stephen Brookfield (2019) describes as a diversity jog where one is working to promote diversity, even though their acts are not generating impact which could be intentional or unintentional.

Another Black, female faculty member mentioned that she is often "asked to do the usual Black History Month stuff" and to speak to Black students regarding recruiting them to their department. Her experience with regard to being called upon to handle 
matters that involve Black events or Black students supports an assumption that one's racial identity essentially prequalifies them, in the eyes of their majority counterparts, to handle those situations. Another core assumption is that Blacks are expected to participate in these activities. An Afro-Latina faculty member also admitted to being "scouted out for diversity events" even though there are plenty of others in her department that can engage with diversity matters, but "they say "no" because they are not interested in doing that. It's not part of their job.”

The ability to say no to participate in diversity-related tasks can be understood as a privilege, and the fact that her counterparts don't understand being active members in promoting diversity to be a part of their jobs means that they, too, understand these task as additional loads with no financial reward that they seek out minorities to spearhead.

One of the Black, male administrators also experienced this kind of cultural taxation throughout his career trajectory. He expressed that he finds himself being, to an extent, typecast into a particular career path stating that:

I'm starting to feel like people want me to be like, excellent in this right? But you know, forcing the [pause] I think, like boxes, you [pause] me [pause] I can only be the director of, you know, multicultural affairs, when that's not maybe the goal I have for myself.

He expressed feeling boxed into a specific category; trapped into being an expert in diversity matters, almost as though he is being directed into a role, because of his race, that he has actively been attempting to grow out of.

Being called upon to educate members of a majority group about diversity also supports the concept of minorities being expected to be experts in diversity matters. This form of cultural taxation surfaced with one participant, who identifies as Black, who 
expressed being utilized as the go-to person for diversity-related matters that correlate with her cultural background in the following statement:

Well, once there's a yearly thing, it's a young African leaders [inaudible] meeting that President Obama set up and it gets younger African leaders from all over Africa and bring them over here and do a leadership workshop for them. And they go from institution to institution. And so, they asked my boss to speak with them, and he was like, 'Hey, you're African,' so I would like you to come with me... So, I think he knew that he only knew so much and [pause] He deferred. They invited me in, I didn't have to be there, and they deferred to me like "You know, you know them, you know how they think, what they are expecting [pause] so lead us, I will follow. -Black, female faculty

In this particular case, the participant was called upon because of her African background. As a result of her boss being unfamiliar with African culture, students, or people in general, he deferred to this particular faculty member to lead and ultimately take over the task. The participant completed the task out of a sense of responsibility. While this action could be beneficial to the guests because the faculty member is someone that they could likely relate to, racially, culturally, or ethnically it does not take away from the fact that she was assigned this task, which her boss was initially assigned to do, because of her ethnic background.

Service

In addition to expectations being understood as an external imposition of cultural taxation are acts of service inflicted by the institution and its dominant constituents. These kinds of services are imposed based on one's racial and or ethnic difference in an effort to satisfy diversity needs. In these cases, individuals are sought out to complete certain tasks that are outside of their scope of duties and often do not include compensation. 
While serving on affirmative action committees, as noted by Amado Padilla, did not surface throughout the interviews, some participants did express instances in which they were called upon to serve on multiple committees to ensure that committees were diverse. Three of the participants presented instances related to this form of cultural taxation. One Black, female faculty expressed that she "Ended up having to be placed on every hiring committee that there [was] to ensure that there's diversity on the committee." The fact that she was placed on multiple hiring committees indicates that the department lacks enough diversity to share the responsibilities being placed on this one faculty member. An Afro-Latina, female faculty also spoke of a colleague of hers that "Always kind of gets pulled in as far as for serving as a diverse member," indicating that her experience is not an isolated one; that she is aware of others in similar spaces encountering similar situations. During one interview, a Black, female faculty referred to herself as "The diversity thing," stating that she gets "put on all the committee's because they need diversity," which is indicative of a shortage of "others." While the overutilization of underrepresented members of the academy did not arise as a part of every participant's experiences, one participant presented the following:

I had seen it, like, university, universities require the diversity of the committee for search committees for the research faculty. So, we have like, one Black guy, and that guy has to be on every freaking committee. I was embarrassed. Like he was on five different committees, and he wasn't even a full-time faculty here, but we were begging him because they were not gonna let us move forward... I was [Position title], I had to go like a jerk... like, "Oh please, we are having this..." Yes, Yes, there is. And then those people are, you know, you don't get paid for that. I can see that happening. -Iranian, female faculty

In the case above, this underrepresented faculty member was perpetuating this form of cultural taxation. She had not personally experienced it; she observed and even, in some 
instances, imposed it upon others at some point in her career at FIU. This sheds light on the idea that people can be aware of their actions, and the implication of their actions yet still continue to put underrepresented members of the academy in these predicaments because of policies put in place by the institution.

Other ways participants expressed partaking in service as an externally imposed form of cultural taxation is by serving as the liaison between the institution and the ethnic community. This type of service can take place even though one may not agree with the institutions' policies. Throughout the interviews, however, this type of cultural taxation was not articulated often. It surfaced with one participant who happens to be a Black, female faculty member. She made the following mentions:

It'll just be anything that has to do with minorities. I mean, I want to say they don't [pause] they don't mean anything by it. But that's [pause] that's a cop-out. ... They're not going to go to Florida Memorial [University] and recruiting [pause] because they're [pause] they might have an issue with the area, the time of day that I'm going, they may not believe that those students are as well prepared.

Here, the participant describes cases in which she has been assigned to and accepted the role as the designated face of diversity for her department. In addition, because of her racial and ethnic background, she is assigned certain tasks that relate to reaching out to other minorities. While this could be seen as a progressive move by the institution, presenting that they do have minorities within their department and that "others" are welcomed, it can also be argued as a magnification of the absence or invisibility of dominant groups in the implementation of diversity. Additionally, the participants' perception of her counterparts as not meaning anything by their actions and assumptions could be understood as a perceived sense of innocence; but this could also create room 
for conversation about how presumed innocence or ignorance could be weaponized in a way to justify certain behaviors. She also describes what could be understood as a lack of comfortability of dominant groups in interacting with minorities.

Being called upon to serve as a translator or interpreter when the institution is presented with non-English speakers did not arise at any point during the study. In an institution like FIU, many of the faculty and staff have the linguistic capital that would accommodate situations if/when non-English speakers required assistance. Furthermore, most of the participants lacked the linguistic capital to act in this capacity. The inability to speak multiple languages, mainly English and Spanish, was expressed to have had a negative influence on many of the participants' experiences within the institution as it relates to work- and social interactions. Four of the participants were multilingual: One was fluent in English and Spanish, one was fluent in English and Portuguese, and two were fluent in English, Haitian Creole, and French. One participant actually conceals her knowledge of another language. When asked if her colleagues have ever called on her to assist with Haitian Creole translations, she stated: "No. Because almost none of them know."

Mentoring, understood as an external force, was also a theme among the participants. Throughout the study, five participants expressed that they lacked mentorship during their time within the institution. Although "lack of mentorship" was expressed, the act of mentoring students did arise as well. One participant expressed the following:

Any Iranian students who would get on campus, they find [me] first. The first week::laughter::... In my department and outside, actually yeah outside. And even, not even before coming to FIU, they would come in 
[pause] you know, they just asked for help. And that's, that's something that I welcome. It has not been like, you know, I won't say it's a burden, but it's definitely extra external responsibilities. -Iranian, female faculty

In an experience like this, not only would one be taxed in accordance with proximity, but also across campus, especially when ones' minority status is magnified by greater disparities. Other participants expressed similar sentiments in which students sought them out specifically for guidance and support. In addition to being sought out by individual students, another way that this kind of cultural taxation can be experienced is by being sought out by student organizations as one Black female administrator expressed that she "was invited once to take a mentorship role with the Haitian Student Organization."

One's tendency/ability to accept or decline these requests could influence their experience. But in some cases, where boundaries are limited or nonexistent, like in the case below, one can find their load to be heavier.

I try to mentor all my students, to be honest- anybody who reaches out. I try to mentor them, and then [pause] and then [pause] I kind of try to look out for the [pause] the [Points to skin]. The ones who I know are struggling. -Black, female faculty.

This particular faculty member has a commitment across racial and ethnic lines.

Although she admits to having a special interest in her black students, she does express being committed and available for any student that reaches out to her.

In addition to mentoring students was the act of mentoring colleagues as one White, male faculty member expressed the following: "I am now mentoring three of my colleagues... It wasn't done for me. And I'm specifically mentoring them for promotion." Even though he lacked mentorship during his career, he has taken on the responsibility of mentoring those rising up behind him. Mentoring has been identified as being key to 
one's professional success. It is also linked to career satisfaction. While some of the participants expressed not having mentorship within the institution, some did and expressed the positive impact it has had on their professional journeys.

\section{Tokenism}

While the study was not exclusively designed to explore tokenism, it did surface with some of the participants. Tokenism, if understood as a symbolic attempt by the institution (an external force) to serve its need for ethnic representation, could be understood as an external imposition of cultural taxation. Four of the participants explicitly expressed feeling as though they were being used as tokens. Three were Black, female faculty, and one was a Black, female administrator. While some instances were perceived, some were blatant and expressed by their colleagues. One of the Black, female faculty members shared the following regarding when she first started in her new position at the institution: "When I finally came to this campus...I got the comments, 'of course, you know, you were hired because they needed somebody Black, we needed a token in the department', that EOPD is down their backs."

Again, in an environment understood as diverse, the need for somebody black could be indicative of an absence, and the participant expressed being told this directly and casually. In addition to being told that her selection was not necessarily because they wanted to hire her or someone Black, but because there were external pressures from FIUs Office of Equal Opportunity Programs and Diversity (EOPD).

Another participant made the following statement regarding her experience with tokenism: 
I've even been told in some cases to apply to job searches so that they'll have a more diverse candidate pool. So sometimes feeling like I'm tapped into as a token so they'll have the [pause] more appearance of diversity. But it can also be insulting at times, because not knowing whether or not they're really interested in the value that I bring or just the fact that I check a box and they have a woman, I'm young, I'm ethnically diverse. -Afro-Latina, female faculty

The participant expressed another practice of creating the illusion of diversity. In this case, she presented perceiving being used to diversify applicant pools for positions in her department. Her experiences with the not so implicit acts also lead her to question the departments' intentions for her selection. A Black, female faculty member stated:

Part of it is the lack of numbers because the thing is, you're so few that you are [pause] you become the token in the old fashioned 60's sense of the word... And when you're the token, you get overworked because you're called on to be "the" person in so many situations...

She articulated her awareness of her situation, along with what it entailed for her during her time at the institution. In another statement made by a Black, female administrator, a participant presented a situation in which she overheard another administrator utilizing her presence to argue that the department was diverse:

I remember hanging up here, hearing a comment made by some senior administrator: "Well, yeah, look, we have someone, we have a Black" because I was the only Black person at that time- And yeah, "we have like, we have a Black... so we're, we're a very mixed environment.

I presented this quote in a subsequent section to support a claim about problematic conceptualizations of diversity; it can also be used to support a claim about the practice of tokenism as the administrator refers to having "a Black" to argue that the environment is diverse. In another example, an Afro-Latina, female faculty member said:

Kind of an example. I guess it was about two years ago. I was recruited to go to Nova Southeastern University. One of the faculty members let it slip that, "Oh, it's a woman and a woman of color. You kind of check all of 
our diversity check." I didn't expect him to actually [pause] I kind of knew that was the case, but the fact that he actually came out and said it.

In some of the cases above, it is not only the existence of tokenism that poses a problem, but the comfortability of others to explicitly express their utilization of the one or few underrepresented individuals in their respective spaces to satisfy diversity requirements. The statements above present how individuals were being utilized to represent and/or attempt to magnify diversity in spaces that were not necessarily diverse.

\section{Internal Impositions}

Unlike external impositions that are initiated by the institution, colleagues, and/ or students, internal impositions of cultural taxation refer to experiences that are initiated by oneself. The participants' experiences relating to internal impositions of cultural taxation revolved around three themes: obligation, coping, and critical agency. In the study, obligations are understood as duties or commitments, and coping is being understood as facing, managing, and dealing with issues one encounter. Critical agency is being understood as one's act of critically assessing and making conscious efforts to reject existing norms (Poveda \& Robert, 2017). Mentoring, in this instance, is being understood as an internal imposition if conceptualized as an obligation.

Obligation

Some of the participants expressed a sense of responsibility when it comes to mentoring or providing support to underrepresented students; this was conveyed specifically by a Black, female faculty member as presented below:

I push, and I use every opportunity to make sure they are doing it right... I have a few of my students that have my cell phone number and [pause] every time they have personal issues, they text me... Deep down, I kind 
of, when I see one (Black student), I feel responsible. I feel responsible, I think. I see a real opportunity that's mine to help lift them up with like letting them know that I'm expecting excellence, or letting them know that if you want me to put away this the professor hat, I can actually sit down and have a conversation, confidentially between you and I because this is who you are and I understand your struggles [pause] to an extent.

Mentoring underrepresented students, and in some cases, other faculty was understood as a moral obligation among some of the participants. Their experiences, coupled with their understanding of the environments they occupy was shown to serve as a motivational factor in their decision to welcome the responsibility of mentoring those coming up behind them even though they themselves may not have had a mentor or the support that they desired during their professional growth.

Another way that participants expressed a sense of obligation was by agreeing to participate in activities that they were scouted out to do. In this instance, there could be an intersection of internal and external impositions, whereas a request may be made externally, but the sense of obligation, duty, or responsibility guides one's willingness to participate in the requests. A Black, female faculty member expressed that she goes out of her way to attend certain events geared toward the Black population because she has a desire "To see a specific group of people" and that she is "Targeting a specific group of people at this point in time, because [she knows] that the students will benefit from seeing people who look like them in the front of the classroom." She articulated that she sees a need to recruit more Blacks in her department and that she commits to these activities because she knows that no one else in her department will - that if she does not carry out the act of advocating for more diversity, it will not happen.

An Afro-Latina faculty member also expressed a similar sentiment: 
We have occasionally diversity events, and I am scouted out for diversity events [pause] and as a physician at a diversity event [pause] I have my data that shows that a lot of the disparities between African-Americans and Whites in health indicators are because of behaviors of physicians... if I don't say it, it never gets said, because everybody is dwelling on what the patients do, which is just ignoring the research, basically...Every time I get taken up to do anything [pause] and I get taken out to do it a lot [pause] because whenever somebody else- I mean, there are other people that can do it here [but] they say "no" because they are not interested in doing that. It's not part of their job. It's part of my calling.

In the above case, the participant understands her experiences as a part of her calling. She recognizes that there are others in her department that are fully capable that are not Black, but they have a tendency to decline the opportunity to engage in conversations or events that have anything to do with diversity. While participating in some additional duties have been considered a burden by some participants, a commitment to change surfaced during the interviews. In these cases, the participant succumbed to what Amado Padilla refers to as cultural obligations. In addition, among the sample, only the Black participants explicitly expressed this sentiment.

In some cases, people may feel obligated to take responsibility for settling problems regarding sociocultural differences among the members of the institution (Padilla, 1994). This form of cultural taxation did not surface often. It did come up frequently with one participant; however, it is important to note that the participant's position within the university warranted these kinds of mediations. So, while this component did not often arise from the participants in the study, the experience shared by the one particular participant shed light on the kinds of socio-cultural issues that arise on campus that could traditionally warrant mediation by the underrepresented members in the academy. Also, the existence of specific offices designed to solve issues on campus, 
including sociocultural issues, may contribute to why others in the study may not feel the need to take on the responsibility. In fact, two of the participants made reference to utilizing the services. An Iranian female faculty stated: "Do the deal, actually go to the EEO, equal employment office, and then talk to their people." A Black, male administrator made the following statement regarding the utilization of campus resources:

So, I ended up talking to [Redacted] about this, and she told me, "No, you don't go complaining." If I go complain, there is backlash. There could be backlash to me. What you do is just talk to them, you let her office know, and her office will handle it.

Referencing the mention of "backlash," fear of retaliation or "backlash" may have also contributed to the lack of data received about this topic - the participants may avoid tackling problems regarding sociocultural differences. I explored this notion in an earlier section where I address powerlessness and a culture of silence. But again, speaking with one of the administrative participants revealed a series of issues pertaining to sociocultural differences at FIU. Some of the issues divulged are presented in a subsequent section addressing coping with problematic behaviors.

Participants expressed practicing, what I refer to as, differential emotional management as an internal imposition linked to obligations; the obligation was generally grounded in fear and feeling responsible for combating stereotypes. Some of the participants expressed sentiments that they did not feel comfortable expressing themselves (particularly in relation to discontent) as freely as their majority counterparts. In one instance, a White, female administrator stated: “I don't speak up. But then my concern is that at the time when I do speak up, I'd be considered offensive." Prior to this statement, the participant explained a series of events and situations in which she either 
felt disrespected or disregarded; so while she felt she was on the receiving end of disrespectful behaviors, she did not feel that she had the freedom to address her colleagues about their actions towards her. This notion was internalized by multiple participants; she was the only non-person of color that expressed these sentiments during the interviews. However, the next section will address a theme that arose among some of the participants of color that influenced their differential emotional management.

A sub-theme that surfaced throughout the study was the "Angry Black Woman/Man Phenomenon.” This concept surfaced throughout some of the interviews several times without inducement. This stereotype has lingered among societies' perceptions of African-American women. The discussions around "the angry Black woman" were heavily aligned with what I argue is another way that underrepresented members of the academy can experience cultural taxation that was not introduced in Padilla's (1994) original work: stereotype deconstruction and differential emotional management. The participants expressed that many of their actions and behaviors in the workplace were self-policed by efforts to avoid satisfying this stereotype. One of the Black, male participants expressed similar sentiments.

Four participants explicitly expressed that their efforts to avoid being "perceived" as the "angry Black woman/man" in the workplace prompted the suppression of their discontent with actions committed by their colleagues and matters in their work environment even though they acknowledge that many of their counterparts did not filter, nor suppress their emotions. Participants spoke of an overall fear of expressing anger even in situations in which they felt it was warranted. One of the Black, female faculty articulated how "People interpret body language" and how, if "you act a certain way, 
you're an angry Black woman.” During an interview with a Black, male administrator, following a discussion about his perceived inability to express discontent within the institution without using some sort of strategy involving anonymity, he expressed:

What am I? Am I the angry Black man now? Am I the upset employee? Am I the difficult employee that's hard to get along with? What am I now? [Pause] I don't want to be the angry Black guy that's standing up for my rights now and the one's in charge get me out of the way so they can continue with their agenda.

This depicts a fear of expressing discontent with presumed unfair treatment out of fear of being judged, stereotyped or fired. The reference to a perceived "agenda" was also something I thought was worth noting. When I inquired further about the "agenda" he referenced the following:

I walk into the Green library [pause] it was the World Cup going on- In the Green library they got the TV on Spanish broadcast... I'm sure they would be happy that the World Cup was on TV in Spanish [pause] all TVs at FIU... [and] How many blacks, how many Haitians, how many of the other races are prevented from working here because they're not bilingual (e. g. speak Spanish as a second language)?

He spoke of what he perceived was an environment that was strategically working to transform the demographics to house mainly, if not only Hispanics and members of the LatinX community, which negates diversity efforts and further creates an environment that induces cultural taxation; this, however, would depend on how one defines or conceptualizes diversity. This participants understanding of diversity was linked to the intersection of race and ethnicity, although, throughout the interview, race seemed to weigh in heavier.

Lechuga (2012) states that the efforts of underrepresented members of the academy to manage their emotions and "remain silent" in some cases "may take a harder 
toll [on them] when derogatory remarks are made about them, their abilities, or other [minorities]" (p. 94) — this was tied to one being "compelled to behave inauthentically so as to conform to the feeling rules of the academe" (p. 94).

Differential emotional management can also be linked to another way underrepresented members of the academy can experience internally imposed forms of cultural taxation: Stereotype Deconstruction. During the interviews, some participants expressed their concerns about stereotypes and how they felt the need to prove that they did not fall into prescribed categories. A White, female administrator expressed the following about assumptions that may be tied to her race and how her race and transcend into the expectations of non-European-Americans in an environment where she is the minority:

And I think when you think of White people you think of like [pause] Republicans or I don't know [pause] people who love country music and Confederate flags, and that's not my case. I'm kind of [pause] I don't care, like, I don't represent [pause] I'm not involved with those types of things. I'm not involved with Republicans; I don't like guns, I don't [pause] I do listen to country music but I also listen to hip hop or rap. So I think the idea of what a White person is, is very different depending on where you're at... And I don't [pause] I don't blame them like [pause] society has put a lot of societal norms for letting them know [pause] [But] like, I'm not what you see on TV or what you assume.

The participant gave examples of how she understood her Whiteness to influence her experience in a non-predominately White environment, specifically, with her co-workers and minority students. She spoke of instances in which she had to work a bit harder to deconstruct certain stereotypes that may have been tied to her race in an effort to build trust with minority students. She also spoke of instances that involved some of her colleagues assuming that she was interested in certain activities or topics and would 
approach her about them, attempting to engage with her about matters that she could not identify with that ultimately would make her feel uncomfortable.

A Black, female faculty member also expressed sentiments of working to deconstruct Haitian stereotypes, stating: "I spent too much time trying to say I don't eat cat, I didn't come here on a boat or on a raft. I don't brush my teeth with sticks. I don't. It was just too much." She expressed that students would make those kinds of inquiries when they discovered that she was Haitian. She also expressed how mentally exhausting it was to have those kinds of conversations which ultimately lead to her concealing her cultural background to an extent, expressing that she "used to have a flag" in her office, but she eventually took it down to reduce the influx of the conversations that accompanied it.

While stereotype deconstruction can influence a person's behavior, it can also impact one's ability to be their authentic self. One Black, male administrative participant had a perceived inability to be his authentic self, which he felt may or may not fall under a "stereotype," stating that: "I'm real funny about like [pause] hanging out with people outside of work or like — Because I don't ever want to be like judged." He limits the potential of his relationships with his colleagues by limiting their interactions to on campus matters only. The act of deconstructing stereotypes that are tied to one's race, in some cases, are grounded in the idea of paving the way for others coming up in the future. A Black, female administrator said the following:

I feel that it's important for me to conduct myself a certain way. That way, I don't have the stereotype. What if I deconstruct this narrative that people wanna usually label us with, whether it's like, "Oh, we're not working enough," or "We're lazy," or that "We're not intelligent," ... all these stereotypes, I always wanna feel like I need to beat them down... I do feel 
like I have a responsibility, every single day, all the time. I would say that can be the taxing part of it, yeah. I think that that can be taxing because you constantly have in the back of your mind that, "Oh, you have to do better because you wanna be this example," right? Not just for me or for the people, but also for the next generation. So if people could stop stereotyping others.

She shared a scenario in which she is conducting stereotype avoidance and deconstruction; it has become a part of her, admitting that it is something that she feels responsible for "every single day, all the time." This can be understood as an added continual pressure to filter one's behavior, which influences the output of every action she performs. Another Black, female administrator shared similar sentiments regarding deconstructing stereotypes, but not only for future minority professionals, but current ones as well; expressing that "When you do something wrong, if you make a mistake, it kind of it reflects on the whole as well," indicating that minorities, in certain cases, lack the privilege of assumed individuality as she also states that people "should judge someone on the merit of what they put in individually, but unfortunately, that's just not how it is [pause] at FIU or in the world."

Emotional suppression can have negative impacts on one's overall social experience. The suppression can lead to flawed perceptions of the experiences of underrepresented members of the academy, which could contribute to a reproduction of the kinds of behaviors that trigger negative emotions. Additionally, emotional suppression can also decrease access to resources designed for social support, lower social satisfaction, and damage relationships in the workplace (Peters et al., 2014). 


\section{Coping}

Another theme that arose during the study that could be understood as an internal imposition of cultural taxation is the process of coping with problematic behaviors that one may encounter as a result of their underrepresented status. These problematic behaviors can stem from colleagues and/ or students. I will be defining a problematic behavior as any action or statement that intentionally or unintentionally has an adverse mental or physical effect on the receiving party. I include the subsequent section describing some of the problematic behaviors participants mentioned in order to set a foundation for the presentation of their coping mechanisms and paint a picture to what some of the underrepresented faculty and administrators present as a part of their experiences. Some of the statements regarding colleagues' problematic behaviors include:

I do have a colleague that he really liked guns, so he would come talk to me about guns, and it made me uncomfortable. So I don't know if he just assumed because of my nationality that I like guns, but he wouldn't talk to the other people in the office about guns. So eventually, I had to sit down and tell him like, I don't like guns, it makes me very uncomfortable when you talk about guns. -White, female administrator

In the case above, the participant expressed a scenario in which she was placed in an uncomfortable situation because a colleague made an assumption about her and her presumed interests. Although she cannot confirm that her race ["Nationality"] is what attracted these conversations, she acknowledges that she is the only White, non-Hispanic employee in her department, which houses multiple people and that he would scout her out to have these conversations with. 
A Black, female faculty member presented the following regarding some of the problematic behaviors she has faced:

The gentleman I was working for said, "There's no way you could possibly be Haitian." And I asked him why. And he said, "Because you come to work on time."... I would get those types of comments on a regular basis... that I was professional, but Haitians and Blacks overall were not... When I finally came to this campus, then I got the comments, of course, "You know, you were hired because they needed somebody Black, we needed a token in the department." That EOPD is down their backs... We had a situation where EOPD was going to put a freeze on the department, a hiring freeze. And one of my colleagues when he heard that - we were literally standing in the chair's office - and he said, "What does that mean, we have to start hiring incompetent people now?" And I literally, like, I was at a loss for words, I didn't know what to say, I could not shake the words... And they feel comfortable telling a Black Ph.D. student, "You'll get a job just because you're Black, because they need to fill a quota." Which is what was told to one of my students.

The above statements provided by the participant presents a series of problematic comments, assumptions, and actions the faculty member experienced in her role at FIU. The comments were made confidently, and without regard to the impact they could have on the receiving party. Additionally, the comments had become a regular part of her experience as they would take place, as she stated: "on a regular basis." Some statements were undermining her credentials and abilities as they could insinuate that her Blackness outweighed what she could bring to the department in their decision to bring her on board, in addition to allowing that thought process to transcend to Black students in the program as well. In the proceeding statement, a Black, female administrator presented:

When I first came here, when the first African-American I met looked at me and told me that had she been on the committee, I would not have been hired because I took the job away from Black-American... I remember when I first started here, I would go to receptions, and if I didn't put my name card on, you know, someone would ask me to get them a drink because they would think that I might have been, you know, part of the serving crew... When I came in there, the job that I got was there was 
someone who wanted the job was literally in my office, and male Cuban, who wanted to just assume that he would get their [position title]. What I found out afterward was that when I [pause] when he didn't get the position, he told people I was a communist.

She presents a series of instances that coincide with what Hirshfield and Joseph (2011) identify as problematic behaviors that minorities sometimes face. In her case, the intersectionality of being Black, and an immigrant impacted her experience with other Blacks in her department as well. Her comments regarding being a part of the "serving crew" were also indicative of assumptions that Blacks occupy lower-level positions within the institution, which can be argued as a problematic conceptualization. With regard to the "communist" scenario, while it is unclear if this was due to her racial/ cultural background, given the demographics of the department, which she stated was mainly Cuban, coupled with the history and impact of communism has had on the experience of Cubans in Cuba, the act of her colleague spreading a rumor like that could further negatively impact her relationships with the rest of her colleagues, who are members of the dominant group.

A Black, male administrator conveyed the following: "I mean, a big part of it is me reminding people that "you are not my boss, and this is not your place." He spoke of how employees in his department on, and below his rank attempt to play the role of his supervisor; this includes actions like delegating work to him, monitoring his work and treating him in a condescending manner. Overall, his colleagues at multiple levels regularly try to have authority over him, which he acknowledges as an isolated case rendered to him only. 
Another participant, an Afro-Latina, female faculty member shared the following: "The only tiny little resentment that I have is this, this bullshit about how I got, I got a ride." She shared a series of incidents that involve her colleagues, particularly White, male colleagues, insinuating that her presence is attributed to Affirmative Action despite her talents, contributions, and credentials. She explained that those assumptions were, at times, accompanied by comments. This would fall under the category of a problematic behavior or problematic assumption.

Another participant shared the following:

It could be challenging, again, you [pause] you tend to have people tell you that something is one way and you know, for a fact, from your personal experience, or just what you've seen through your eyes of being a minority, that that's not the case, and that it's something else. And they either don't understand or don't want to understand. Can't understand. So, it's challenging. - Black, female faculty

The participant explains a situation that Hirshfield and Joseph (2011) present about dealing with colleagues who deny racism or discrimination. When experiences are made out to be a phantom concern, it becomes difficult to mediate them. The statement below shares a similar sentiment; The Black, female administrator explains how in mediating cases on campus, she encounters a number of employees that try to deflect from the possibility that their conflicts are or could be race-related:

People that you're speaking with, just saying things like, you know, "Let's not make this about color." You know what I mean? In a meeting, it's like, it's like you, you're not making it about color, we have to explore the possibility that it is that [pause] not only about that, but that is going into it, and that's just the reality of that. So things like that can get frustrating. Feeling like you're making it up, or you're just being sensitive, because you're a Black woman - like no I'm not being sensitive, we just understand it in a way that you can't... And you're going to get challenges getting the position, you're going to get challenges keeping the position, 
people respecting you in the position, listening to you [pause] getting promoted from those positions, you know, it's, it's, yeah.

In addition to being confronted with the denial of racism or discrimination, the previous statement also brings to light the issues members of minority groups could face during their career trajectory, which surfaced among multiple participants across race and ethnicity. Another problematic behavior that can simultaneously be tied to expectations is having one's work be accredited to someone else's efforts. In the statement below, a participant expressed how assumptions have been embedded in her experience as an underrepresented faculty member:

Sometimes when people see you in places where they don't expect to see people like you [pause] They are dismissive...I have found that there are some people who like to ride on other people's curtails; trust me, it's not the Black ones and that kind of annoys me. I am a team player. I will do anything for the team... So there's this non-colored person in here [and people assume] he might be the leader. And then they expect [that he is the leader] and then the person can just take credit for it, and they do it. I've had that happen many times.

-Black, female faculty

Some of the assumptions made by the dominant group in her department can be tied to biases that create a space for problematic behaviors that she has to spend time dealing with or coping with. The same professor also referred to an ongoing situation in which some students and faculty confuse her with another Black professor even though she expressed that they look nothing alike. She stated: "I kind of find it amusing...they confuse [pause] they kind of have us confused sometimes. Like [pause] a lot of them [pause] like Dr. [name of another Black professor]. That's a challenge.” I personally looked up the other professor and confirmed that they do not even remotely resemble one another. This kind of behavior confirms studies that investigate "cross-race effect" 
(Hehman et al., 2010; Pezdek et al., 2003; Sporer, 2001), which argues that some people have a cross-race recognition deficit which makes it challenging to differentiate members from other racial groups. The same professor also stated:

They were like, "Hey, you need to get your license." And I'm like, "No." And then [they were like] like, "Yes, yes." And somebody said to me, "Hey, you remember you're Nigerian, right? They will think you're just faking; you don't have it." And I'm like, 'Hey, okay, that's true. -Black, female faculty

The above statement has ties to stereotypes about one's nationality and how they can lead to assumptions and unwarranted treatment. The participant did eventually obtain the license they were referring to. A Black, male administrator mentioned:

I was just work, work, work. I would be quiet. Blacks stuff would come up. "[Participant quotes a colleague]: "Don't you think all Black people should go back to Africa?!" I'd sit there like I ain't hear it and because I had to kiss ass before... so I just work, work. A year later, all the White folks: "Hey [participants' name]'s a good Black person." "[participants name] don't complain.

In the instance above, the participant expressed situations in which he's had to suppress his feelings and comments because he had to "kiss ass before [he] can kick ass" indicating that he had to tolerate certain behaviors within his environment to gain acceptance, and avoid being known as the "angry black man" so that when he does report someone's behavior:

It holds more weight because the first two or three years all you saw was [his] work. You knew [he] was good. So for [participants name] to say something you must have really really struck a nerve. You must've really did something wrong because [participants' name] has taken so much stuff before. We know he's a good guy.

Another concern that arose was the lack of empathy from colleagues when social issues took place- typically grounded in racial injustices. A Black, male administrator stated that 
"When there are tragedies that occur, you know what I'm saying, they usually kind of [pause] How can you say [pause] give a nurturing feeling, if it's Hispanic-related, but if it's African-American, not so much." This could indicate an inability to relate to members of other racial or cultural groups. The potential inability to identify with others can impede on one's ability to empathize with them, hence creating an environment that can make underrepresented members of the academy feel isolated and not valued.

The same participant also said:

"I think FIU leans to hiring their own, and when I mean their own is people who understand their culture, who understand their vision, and who understand their community. As being a hiring manager on the board for my college, the last eight hires have been Hispanic, and I'm not trying to [pause] maybe I'm being biased. Who knows? But sometimes the candidate wasn't always the best candidate, but because they were Hispanic, and they fit in with the culture, they were chosen, and for me, that always raises an eyebrow. You can't move forward if you keep doing the same thing over and over.

This could speak to what some may argue as potentially problematic hiring practices, especially if there are people from other racial/cultural groups, who are better qualified to fill positions; it also could negate diversity initiatives. In a situation like the one presented, the dominant and underrepresented groups remain at their current status, which provides a space that contributes to the continuation of cultural taxation. But, it is can also be argued as a relevant hiring practice that contributes to having a staff that reflects the student population that may better serve the needs of the particular population.

A Black, female administrator also expressed: "I remember a time that it was really frustrating. Because every time that I started to talk, it was just like, 'you don't have anything to say." This describes a case in which underrepresented members of the 
academy may face situations that indicate that their voices or input are not valued, which too could be linked to stereotypes and assumptions. She also made mention of being the go-to person for tasks that her counterparts would reject. She stated: "They would feel like I am more pliable than the others. So to avoid confrontations with the others that don't look like me, they go to me." This, too, can be linked to the concept of stereotype deconstruction and working to avoid being classified as an angry Black woman. She expressed, essentially, being the go-to person for duties that her counterparts considered beneath them, which could speak to their perception of her as well.

Problematic conceptualizations of diversity also arose as a Black, female administrator shared the following:

I remember hanging up here, hearing a comment made by some senior administrator: "Well, yeah, look, we have someone, we have a Black" ... I was the only Black person at that time. And yeah, "we have like, we have a Black [pause] so we're, we're a very mixed environment."

The behaviors presented in this section include colleagues ignoring and/or minimizing race issues in department relations, participants being used by colleagues as marketing tools to represent "diversity," ultimately being tokenized, being met with racist and/ or stereotypical comments, facing implications that faculty/administrators of color do not belong, and dealing with colleagues who deny racism or discrimination (Hirshfield \& Joseph, 2011).

In addition to coping with colleagues, problematic behaviors was the act of coping with student's problematic behaviors. Previous studies highlighted coping with colleagues' problematic behaviors; however, the study also presented instances in which 
faculty and administrators demonstrated having to cope with students' problematic behaviors as presented below. A Black, female faculty member stated the following:

The students would come in here and ask me, where did I buy my diplomas from? Because I could have never earned all of them... Like, I have five degrees on my wall. And even the Black students question, where did I buy them from? Because I couldn't have possibly earned them?

While the inquiry could be an attempt to start a conversation with the professor- an icebreaker, it could also present the presence of implicit biases some students may have. It could also be an indication of their inability to conceptualize a Black woman with so many educational credentials. It also speaks to the disparity of Black women in her position, with her extensive educational history. Nevertheless, for anyone to inquire about where someone purchased their degrees undermines the efforts the individual under question put forth to get where they are. It can also be insulting and inappropriate coming from anyone, but it is amplified coming from a student, a subordinate.

The same professor also stated that: "The Hispanic students are more vocal about it and their perceptions and their [pause] their beliefs [pause] and tend not to give what I say a lot of credence anyway." The previous quote was concerning her experience in the classroom. She spoke of class discussions and how she observed that her Hispanic students were very upfront about their biases. One example she gave was the following scenario:

So I had in a [specific] class - I was asking why are Blacks and Browns over-represented in the criminal justice system? And none of my Black students had anything to say. But a Hispanic student did eventually volunteer a response, which was [pause] this young man told us, like, "I can't speak for Hispanics. But for Blacks, it's because they're not raised 
with proper values." And I asked him to elaborate. And he said, "Well, they don't believe in marriage, they have kids, and they leave them behind. They're not hard workers..." I mean, gave me a long list... And so I have to maintain my composure [pause] because the problem with being Black, when you're talking about race, and ethnicity is that they expect you to be a subject matter expert, because Black, but they also say you cannot possibly be objective because you're Black. So I had to maintain my composure and with this group of 50, and just ask them to continue to elaborate.

What the above statement describes is a scenario in which an underrepresented professor had to carry the "emotional labor" (Hochschild, 1983) of not reacting the way she may have wanted to in a situation, and why she conducted herself in that manner. She presents the double-edged sword of being expected to be an expert in diversity matters but also being expected to lack objectivity when it comes to diversity matters. But, ultimately, what her statement provides is a view of some of the problematic viewpoints some of the dominant student groups may have about people that look like her and how one would have to manage the situation. A final statement presented by the same faculty member regarding problematic behaviors exhibited by students include students not acknowledging her credentials:

I can't tell you how much time I waste during the day, telling students do not address me by my first name. You don't address my colleagues by their first names, do not address me by my first name. And so they'll go from emails that say hey, [participants name] to [participants first name]. Even though the signature line on the bottom of the email they receive says, Dr. [participants' name].

The students she encountered don't give her the same level of respect as her counterparts. This could be understood differently if the students addressed the other professors in the same fashion, but she indicates that her situation is an isolated one. The notion of her 
having to correct students being a waste of time qualifies it as a form of cultural taxation as it is grounded in the concept of the phenomenon warranting additional time and energy of underrepresented members of the academy.

There were also instances presented in which students would undermine and/or underestimate underrepresented members of the academy's' intelligence. One participant stated: "There were times when I'd be at the [work location] when someone White came up and saw me at the desk and approach the desk said, 'You may not know what I'm talking about, but...' and ask the question." This Black, female faculty member shared that she encountered students that would assume that she would not be informed about a subject matter and preface their question by admitting that they assumed she would not know what they were talking about.

Another faculty member also shared one of her experiences in which a student undermined her intelligence and ultimately challenged her presence in that particular academic space. She stated:

For him to imply that I, who's trying to help him, had gotten my position [pause] taken the position from a White man. To me, the assumption is that everything that I get is I'm actually taking up the space from White men... The student, White guy, who apparently had struggled getting into medical school, he was doing a [degree-type] possibly to try again, you know? And he was talking to, not talking to me but talking to the other students: "She doesn't know what she's talking about. Look, this is this is an amino acid." [Pause] Yes, it has an amino group [inaudible] nucleic acid is a nuclear - I laughed a moment. All I could say was [pause] it is a heavy burden. All this melanin is a heavy burden. -Afro-Latina, female faculty 
Despite the participants' roles on campus, some of them expressed instances in which students' behaviors towards them could have been indicative of prejudice, implicit biases, or even racism. This poses potential issues as the students are in an academic space, a space of reproduction - the reproduction of knowledge and behaviors. The presence of these types of problematic behaviors could serve as a platform for the reproduction of the very circumstances some of the underrepresented faculty and administrators are working to deconstruct. Additionally, the students are the future, and one day they, too, can be in positions of leadership henceforth, creating a continual cycle of problematic behaviors.

In dealing with their colleagues' and students' problematic behaviors, eleven of the participants explained that they had to learn to cope somehow. Some by developing "a thick skin" or putting "blinders" on in order to focus on their path. With regard to various coping mechanisms, participants presented the following:

I've been through so much, I have seen a lot. I've experienced wealth and poverty. I've seen a lot. And so that makes me not take myself seriously. That makes me [pause] I don't read too much into it. I just let things roll... I don't over-interpret stuff. I don't, even when something sounds rude. -Black, female faculty

The aforementioned statement shows how one may take a more reclusive approach to coping by attempting not to allow their circumstance or negative encounters to weigh down too heavily on them; this entails not taking the time to analyze and internalize instances that one may encounter. This could be understood as an avoidance tactic. Similar to the avoidance approach is the act of flowing through the workday with little regard to what is taking place in one's proximity as one Black, male administrator expressed the following: 
At the end of the day, I don't think you really cope. I think it's more so you go through the motions. You deal with it from 8:30 to 5:00, and it turns off, and then when you come back in, it turns back on.

This could indicate, perhaps, the absence of a coping mechanism, and the presence of a more performative approach to getting through the workday. Others, a Black, female faculty member, and a Black, male administrator, took a different approach by admitting to the utilization of alcoholic substances to cope with their experiences. Another coping mechanism that surfaced during the study was the use of faith and religion. A black, male administrator expressed the following:

I have a mentality and my own ideas, but you try to be humble, and you pray to God to put the right words into your mouth and you forgive and forget. You pray to God. I'm a God-fearing individual; I pray. I'm not perfect like Jesus was, but I pray. I pray to God, and then you fight.

Finding humor also surfaced as a coping mechanism as a White, female faculty revealed the following:

I personally chose to have a sense of humor about things [pause] joke a lot. Humor, I use it to make light of situations in a way to connect with people, hopefully. Humor gives me a perspective that lightens the situation.

She understands some of her encounters as serious transgressions; however, she acknowledges her ability to choose how she responds to instances that would otherwise upset her. She also acknowledges the presence of a disconnect between her and her colleagues and uses her humor as a way to mend the divide.

Energy transference also served as a coping mechanism as one of the participants expressed the following:

Whenever I start feeling that way, that, "Oh, I have to do this because people are gonna think that I'm not intelligent enough"...I sort of repel that thought and try to do it for me because I want to see how much I can accomplish... Reverse that, right? Because otherwise, I'm gonna go crazy 
and I'm gonna stress myself. I don't want that kind of stress. That's usually my thing.

- Black, female administrator

In a case like the one above, the participant would mentally adjust the way she conceptualizes an experience in order to avoid upsetting or stressing herself out, thereby transferring negative energy to neutral or positive energy.

Prior studies showed that faculty of color developed coping skills ranging from confrontation to avoidance (Mahoney et al., 2008; Utsey et al., 2000). The participants in the study presented a series of coping mechanisms that included: energy transformation, faith and religion, finding humor, alcohol consumption, and avoidance. Rarely was confrontation expressed as a means. In fact, confrontation avoidance was a common theme that was explored in previous sections of this dissertation, addressing powerlessness. The act of coping also shows how cultural taxation can transcend across spaces- from one's workspace to their home, whereas not all coping strategies take place on campus as many of the participants carry their situations home with them.

\section{Critical Agency}

Another way that one can experience an internal imposition of cultural taxation is through the desire and act of practicing critical agency. Six of the participants expressed sentiments that align with using or allowing the environment, and some of these culturally taxing activities to serve as a stage for critical agency. One participant stated:

That that just two hours that I spent [at Florida Memorial University] changed my entire perception of FIU and my role at the university, and made me feel like I needed to do more. Not [pause] not for all of my students, but I had an obligation to the Black students at FIU that I had failed to fulfill... I am the person, I have to fight for the students, and 
what's right for them... I [also] have to fight for us to hire somebody Black. -Black, female faculty Having had observed so many Black professionals at a nearby Historically Black Institution and realizing the emotional influence it had on her magnified the absence of Blackness in her department- as she is the only one. It also catalyzed her desire to do more for her students and strive to recruit more Blacks in her department to aid in their support and success as her experiences within her department led her to believe that she could not depend on her colleagues to do that type of work. Throughout the interview, she provided instances in which she was exposed to her colleagues' perceptions of Blacks and spoke on situations about how Black students were confronted with statements from faculty about their career trajectory and how they can expect to get hired somewhere to fulfill diversity requirements.

Another Black, female faculty member said the following:

I learned how to maneuver through it. And so, which is why I know. Even though I'm leaving, one of the things I'm doing with the women who work with me, is that I'm making sure that all of them get the education and training and the support that they need. So, when I leave...

The statement aforementioned was regarding the lack of diversity in upper-level leadership in the participants' department. As a result, as she nears retirement, she has been making conscious efforts to train other underrepresented colleagues so that they can be equipped to take over her role when she departs. She is working to reject the norms of her department by strategically training underrepresented protégés that can effectively compete for higher roles. 
When an Iranian, female faculty member was asked about her perception of some of the additional responsibilities she is tasked with, she stated the following: "I see them as positive like giving back to my own community. I don't see any negative. They just they have a little bit of tax on my time. Definitely. But then, you know, man, I make it work." This presents how the concept of community and giving back influences one's behavior and willingness to participate in some activities despite any time constrictions one may face.

Other participants made the following statements regarding giving back and rejecting norms:

If I weren't what I am, I would not feel like I need to do what I do. And that is [pause] many people would say that "It's your problem." ...But [pause] but those are obligations I also seek, because who's going to do it, you know? It needs to be done. And I'm the one to do it and much better, much more. Anything about taking students to the community, to do real community organizing in communities of color pause] I have to do it [pause] because in part because it's expected of me. -Afro-Latina, female faculty

The case above illustrates how one can intentionally seek to participate in activities that will enhance communities of color. The fact that she attributes "what she is" to what she feels like she "needs" to do could also provide a degree of understanding to why some members of dominant groups may not seek these activities; this may be attributed to an inability to identify with minorities, which may contribute to reduced empathy and understanding. This is not to say that one has to be a member of any particular racial/ cultural/ or ethnic group in order to have a desire to promote positive change within. It serves to show a connection between identity and obligations imposed from within. 
Another participant shared the struggles she experienced during her journey and how she works to help students that she identifies with racially succeed in her particular field as she expressed the following:

I kind of try to look out for the [pause] the [Points to skin]... The ones who I know are struggling...I push, and I use every opportunity to make sure they are doing it right. Sometimes it might be perceived as being hard, but it's not. But I know what I'm doing, and I try to kind of have an open-door policy. You need to talk to me; I'm here... I have a few of my students that have my cell phone number [pause] and if every time they have personal issues, they text me... Deep down, I kind of, when I see one (Black student), I feel responsible. I feel responsible, I think. I see a real opportunity that's mine to help lift them up with like letting them know that I'm expecting excellence, or letting them know that if you want me to put away this the professor hat, I can actually sit down and have a conversation, confidentially between you and I because this is who you are and I understand your struggles [pause] to an extent. -Black, female faculty

She uses her position to help change the norms in her field, which does not house many Blacks. Her support aids students in the successful completion of the program and then, ultimately, matriculation into the professional field. She spoke of some of the systemic barriers that she encountered during her academic and professional career, which often influence her desire to assist Black students as a way to combat some of the barriers they may face.

A Black, female administrator expressed feeling responsible for participating in certain activities. Essentially, she observed a need that she felt warranted action on her behalf, stating: "I felt that I needed to contribute, and I wanted to give my time for that...We tend to feel like we're responsible because that's what we're called to do." She 
presents the presence of internal pressures but also attributes her behavior to a "calling," which could indicate a sense of inevitability.

These participants have been able to critically assess issues that not only themselves but also those rising behind them face. As a result, they present intentionally participating in some "culturally taxing" activities as a way to reject existing norms and values. Their shared experiences are consistent with Chang et al.'s (2016) study that explains that some individuals have a desire to serve on diversity-related committees or assignments (Chang et al., 2016).

\section{Social Impositions}

While external impositions are initiated by the institution, colleagues, and/ or students, and internal impositions are initiated by oneself, social impositions of cultural taxation are those that are induced by the social climate of the institution. Many of the participants' experiences regarding social impositions mostly revolved around integration. The participants' racial, cultural, or ethnic difference creates a social disconnect that was shown in the study to warrant extra efforts in order to become, or at minimum, feel as though they are socially connected to the institution. This is an alternative conceptualization of the phenomenon and is being argued as a form of cultural taxation, again, due to the additional time and/ or energy required of one to commit the actions necessary to integrate themselves into the environment.

Ones efforts to combat social isolation are an act that could positively influence one's experience at an institution where they are a demographic anomaly and ultimately influence their decision to remain a member of the institution, which would allow them to continue to serve the institutions need for ethnic representation. 
Integration

Integration, as a theme, surfaced in different ways for the participants.

Acculturation arose as a part of some of the participants' experiences. Some expressed instances in which they felt the need to adjust socially, culturally, and in some instances, linguistically. One participant stated the following:

But like, the point is, like, I'm so concerned about, like, being [his name], I'm from the hood, you know [pause] and I think some things about us come out, and I don't ever want to be taken out of some of the votes for something because I have the authentic moment, you know, so [pause] I just, I think I just struggle to fit in. I just [pause] I don't identify with the culture. And I think that culture has a heavy presence in the workplace. And I mean, we work for Student Affairs, man like you [pause] I feel like you can't be you. -Black, male administrator

This aforementioned statement presented by a Black, male administrator presents how he feels as though he cannot be himself in his respective workplace, even though the people around him seem to have the privilege of being themselves as another Black, female administrator also noted. However, this particular statement could be argued under the understanding that work is performative. Bell (1999) argues that constructions of belonging are grounded in the sense of performance; shaping one's behavior to fit into specific social and cultural spaces connects them to the specific community. The concept of being "taken out of some of the votes" despite his academic and professional qualifications because he has an "authentic moment" could lend itself to the notion that being a "fit" socially, in some cases, can influence one's career trajectory more than their aptitude. 
Another participant shared the following: "It was difficult. I would change my dialect. I would change my conversation. I was doing stuff like that to make myself more acceptable..." This was presented by a Black, male administrator. The reference to acceptability presupposes a sense of unacceptability. This presents a case in which one would have to bounce between being in a state of authenticity and being in a state of performativity, which the participant admitted was not an easy task. This would entail policing one's words and actions in order to connect with their peers. The same Black, male administrator also stated: "Fine, speak your languages. But now you're going to make me learn your language too while I'm at work? Those are some of my experiences." The participant spoke of several instances that he felt forced to speak in Spanish in order to effectively communicate with some members of the university community; cases where he would speak to some members of the FIU community in English and, in turn, be corrected and essentially directed to speak in Spanish. When inquired about any advice he would give to any underrepresented faculty and administrators, he said: "Don't let this university try to constrict you or change your culture you know." This magnified the pressures one may feel to acculturate.

In addition, another participant presented that:

I think the biggest expectation, and [pause] Yeah. I'll say it. Conform. Conform. There's a culture here that's set. You have to conform to it... The cultural stuff, like I said before, it's a Hispanic culture. Everything is revolved around the culture here at FIU in the workplace. You have to conform to it. Otherwise, it's very hard to build relationships here. It's very hard... I learned what the culture was like after a couple of months, I just adapted to it, and that's all you can do because every place has its culture, every place has its ground rules, and you either adapt to it, or you leave. That's just how it is. Unfortunately, I've seen quite a few people 
leave. Non-Hispanic. They leave because they can't relate. Maybe it's because of comfortability. Maybe it's because of not liking the culture itself. Who knows? But I think it makes it harder when you don't feel included... I don't think FIU realizes how strong culture is in the workplace. I understand they understand how it is outside the workplace, but in the workplace, how strong it is and how it really can affect someone, how they work, how they interact, how they see things, how they view the university. I've heard people leave the university because of the fact that they feel like they don't fit in the culture, and you lose good employees that way. -Black, male administrator

The participant in the above statement articulated the prominence of conformability within the institution. He highlights what he perceives as a strong cultural presence within the university that could come off as intolerant of "others," and that if one wants to remain a member of the institutions' faculty and/ or administrative rank, one would have to adapt to the culture of the dominant group. The same administrator also expressed that: "You have to be willing to learn the culture and learn the language itself. Otherwise, you can feel like an outsider very easily." This highlights the influence of cultural norms and language on one's ability to make connections within the university.

A Black, female faculty member described the act of code/ culture switching:

So there are, there are sacrifices in terms of ... not being genuine, not being true to who you are. Because you have to fit in a different mold, it's [pause] it's like being a chameleon. You have to know which skin to show depending on the time of day, the location that you're at. I mean, it's not fun. But that that's essentially what it is. You almost develop like a split personality disorder. Because with one group of people, you're one way with another group of people, you're another way with another group of people, you're another way. That ... takes a tremendous toll.

The participant expressed being in a complex state of performativity in the workplace; this performance turns into a foundation that will influence many of one's behaviors. In this instance, one would be continually processing, policing, and filtering themselves in 
an effort to integrate themselves better, while also serving as a representative for their race. I don't necessarily have or know of an algorithm that could be used to quantify the amount of time it takes to process, police, or filter one's behaviors constantly, but I do argue that it does require additional energy to perform these acts as the same participant explained that "It gets tiring playing a role as somebody else."

The experiences shared by the participants are coherent with LaFromboise's model of bicultural competence. They presented being in an environment where they felt the need to participate in second-culture acquisition in order to "'fit' into the department and be a good colleague" (Trower, 2003, p.6).

Another way that underrepresented members of the academy can experience socially imposed forms of cultural taxation is by the act of managing communication and language barriers. A number of participants expressed communication and language barriers within the institution. Their experiences were in relation to their workspaces, social spaces, and in some cases when it came to communicating with service workers. None of the participants expressed frustration with other languages outside of their linguistic capacity being spoken as that would, in itself, be xenophobic and an extremely problematic way of thinking. It would also negate diversity efforts within the institution. However, participants did express instances in which their inability to speak Spanish as a second, third, or in some cases fourth language did impact them in relation to work, workplace relationships, and navigating the campus. Because many of their counterparts embodied the linguistic capital to communicate in both English and Spanish, some of the participants sometimes found themselves to be at a disadvantage with regard to social and work relations. This disadvantage, rooted in their cultural/ethnic difference, often put 
some of them in situations in which they felt the need to either attempt to learn Spanish, find alternative ways to integrate themselves with their colleagues, and/ or seek ways to cope with the exclusion. The additional efforts it takes to manage communication and language barriers are argued as a form of cultural taxation as it relates to the extra time and energy expelled in order to become and remain an integral part of the campus community — The necessity of these actions are grounded in one's ethnic/cultural difference. I would like to warrant that the interview protocol did not mention or allude to anything related to language; the participants initiated the instances presented in the study.

When asked about challenges that underrepresented members of the FIU community face, one Black, male administrator expressed: "There could be communication challenges you know?" Throughout the interview, he made mention of how his inability to speak Spanish impacted his day-to-day interactions with some staff members. One participant expressed that "one of [her] challenges... is not speaking Spanish.” She stated:

I went to a cultural sensitivity training, and they mentioned like, English is the [pause] the language of the university. That's what the courses are taught in, and that's what the business is expected to occur in. But oftentimes, you see that people are speaking in Spanish. And some days, I will wonder, "What are they talking about?" "Is it relevant to what I'm doing in my office?" "Should I be able to understand what they're saying?" But I can't. So I think ever since I went to that cultural awareness training and they said that business should be handled in English, to make sure that nobody's at a disadvantage. [Pause] That's when it really came to my attention. -White, female administrator

She expressed working in an area where there is an absence of a "common" language and the mental toll it takes on her at times, in addition to how it influences her experience in 
the workplace. A situation like the one she mentioned could make someone feel insecure and potentially distracted because they are regularly trying to decipher if the conversations in their proximity are ones they should be a part of, as some participants did mention the use of Spanish to conduct work matters. It also led some participants to wonder if they are the topic of conversation but are unable to defend themselves because they can't understand the language.

Another Black, female faculty presented that during work meetings: "I would come here, and staff were speaking in Spanish and wouldn't acknowledge me, and that infuriated me." Her inability to speak Spanish, among her bilingual counterparts, made her an outcast. This is not to say that her counterparts must speak English, or that they owe her acknowledgment; it does, however, say that her counterparts may lack a degree of regard for her presence, or perhaps even a lack of awareness with regard to the impact of their actions; but this all would embed itself in the intentionality of their actions which the participant and I are unable to confirm or deny. Her expression of infuriation presents how these kinds of encounters can be emotionally taxing.

In another instance, one White, male faculty member describes some daily tasks/encounters with staff by expressing the following: "Look at having an unexpected occasion to try to make myself understood in the language I don't speak." In those instances, he expressed:

And if one doesn't speak [Spanish] well, or at all the language, the person they're trying to communicate with [pause] we [pause] you know, we have to employ other ways that [pause] communication, like using our hands or signs or things like that. 
This instance presents a moment in which one would have to incorporate alternative means of communication in order to accomplish a task; this would require more time and energy from the individual that could take time away from other work-related responsibilities.

A Black, male administrator mentioned:

I have noticed that if you're not of Hispanic heritage or culture, background [pause] the culture itself in the workplace is maybe a little bit harder for someone who's of a different [ethnic] background because of the understanding of the language and the way things are done... What most people can agree on is that not everyone speaks Spanish. When you're in the workplace, and you have a meeting such a conference room meeting, and you speak a Hispanic language, you know what I'm saying, that shows that there's really not an awareness of other individuals who may not understand the language... I think it's just something with FIU, it's been based on the Hispanic community and the culture that they're in, but I think what they have forgotten also is that being that they are an international university, they have to be able to, I guess, gravitate and tend towards those who also may not be of the culture themselves. They say they're worlds ahead, but sometimes I question that.

In the above quote, the participant made reference to work meetings within his department where his bilingual (English and Spanish) colleagues would use Spanish to conduct meetings in an environment where it was not the language understood by all parties involved. The above statement highlights how one's cultural and/ or ethnic difference among a dominant group can impact work relations in the academy. The lack of awareness or regard triggered negative emotions from the participant impacted by the behaviors. Managing these situations could be emotionally laboring. A White, female faulty member stated: 
I think it's mostly a language thing. I find it taxing that it's hard to be part of a group that you can't fully communicate with... When there are just meetings, and mostly when people speak Spanish, I feel like it's just, to me, it says, "You're not part of this.

This participant made several references of being excluded in the workplace. In this particular case, I actually had the opportunity to observe this happen on campus. She and two other White, Hispanic male faculty were having a conversation when, midconversation, the two men switched to communicating in Spanish, and she was immediately excluded from a conversation she was just actively a part of. The idea of one's challenge, "mostly" being "a language thing," emphasizes the power of language and its ability to influence one's experience in a space where they don't speak the language of power.

In reference to De Kadt's (1993) argument on language and power, the language, in this case, is Spanish, and FIU, in essence, is the society. The functions of the use of Spanish, are communicatory as it relates to workforce operations and social interactions, but also exclusionary as it also relates to workforce operations and social interactions. The use of Spanish is only being argued as exclusive in cases in which bilingual (English and Spanish) faculty and staff make conscious decisions to utilize Spanish in settings that should/could include all present (including unilingual; English) stakeholders. The argument of "exclusivity" does not include the use of Spanish by individuals that only speak Spanish; this argument only applies in cases that involve individuals with the language capital to speak both Spanish and English, but consciously chose not to use the "common" language in a diverse setting, especially when the conversation is workrelated. 
The use of Spanish within the institution could, in some cases, be viewed as a form of resistance. However, simultaneously, the use of Spanish for operational purposes in an environment where it is not the common, unifying language could impact university operations, and, as expressed by some of the participants in the study, one's overall experience and satisfaction in the workplace. The participants in the study generally expressed being open to language diversity, but also expressed the effect the diversity had on their work relations and personal relationships which commonly led them to consciously and/ or unconsciously commit energy towards creating connections with members of the dominant group despite pushback.

Participants generally expressed managing communication and language barriers as a result of their difference and lack of linguistic capital in the workplace. Some expressed attempts to learn basic communicative Spanish while in the workplace, which could add an additional challenge to their experience.

Forced Social Integration, too, surfaced as a socially imposed form of cultural taxation. As a result of isolation, some participants expressed that they have had to force their way into being included in activities and environments on campus. Some of the participants presented the following:

I think there are moments where I feel like [pause] not included, I think there are moments where I see a culture that I'm not a part of that's not as inclusive as it may seem and trying to find a way to connect with that. -Black, male administrator

The participant above expressed that he senses a degree of cultural exclusivity within the institution. His understanding of the environment, for him, warrants additional efforts from himself and other underrepresented parties to integrate themselves within the 
university community. A White, male faculty member mentioned that he's "going to be part of the community too, no matter what," which could indicate the presence of rejection and resistance from the dominant group as it relates to social interaction, but also a degree of counter-resistance as he pushes against the rejection to become socially connected. A Black, female administrator presented that she regularly tries to connect with members of the dominant group stating that: "I'll have lunch with people from different backgrounds and different offices... I try to integrate myself into it, you know." "It" being the social climate that she understood to be uninviting. Another participant spoke of a social environment that did not feel tolerant of "others":

I feel like I am part of the community. I feel like I'm part of the community because I don't see barriers for me because I feel like I work hard to integrate myself [pause] even when I'm uninvited. That's the interesting part. Although I feel like I'm not included...I make myself. I go beyond my boundaries, I think. I'm a rebel. -Black, female administrator

The language she used is indicative of some form of struggle. Having to "work hard" to socially integrate oneself because of their cultural, ethnic, or racial difference presents an additional exertion of one's time and energy to feel like a part of the institution's social atmosphere. One White, female faculty member referenced a cultural "inner circle" that she finds herself trying to prove that she can be a part of but stated her efforts "[don't] work in [her] sense."

The additional efforts relegated to integrating oneself into a space that is rejecting has the potential to create more significant setbacks for the underrepresented individuals. The time and energy spent on attempting to integrate with the institutions' social/organizational fabric could take away from efforts related to their job, which could 
potentially influence ones' road to tenure and promotion. I present this understanding with the awareness that integrating oneself, regardless of their connection to, or disconnection from the dominant group requires some degree of work. The significance behind these scenarios is the resistance the participant experience in the process of trying to connect and integrate themselves.

The experiences shared in the study require both time and energy of the participants in excess of their prescribed job duties; hence, why I argue them as being form of cultural taxation. Their efforts aid in their ability to prevail and continue to serve the institution and its students. As it stands, while many continue to prevail, two of the participants departed from the institution since the interviews to find a better "fit."

\section{Additional Potential Impact of Cultural Taxation}

Cultural taxation has been linked to over-commitment, career progress impediments, reduced job satisfaction, feelings of isolation and burnout (Mahoney et al., 2008; Hirshfield \& Joseph, 2011; Pololi et al., 2010; Hassouneh, et. al., 2014; Mahoney, et al., 2008; Kolade, 2016; Trower, 2003; Blackburn \& Lawrence, 1995). While isolation has been noted as a consequence of cultural taxation, I argue that while it is a consequence, it can also serve a catalyst for what I argue is a newly surfaced form of cultural taxation: forced integration. Some of the participants in the study shared instances that suggest that they do feel isolated; however, they are committed to integrating themselves into their respective environments.

During interviews, some participants referred to self-reflections, where they would question themselves and what they thought themselves to be in the midst of their experiences. I equate these instances to an additional, newly surfaced consequence of 
cultural taxation that I refer to as psyche transformation, which I link to self-concept.

Some of the participants made the following statements regarding some of their experiences:

It, it makes you feel some kind of way... We had a situation where EOPD was going to put a freeze on the department, a hiring freeze. And one of my colleagues [pause] he said, "What does that mean, we have to start hiring incompetent people now?" And I literally, like, I was at a loss for words, I didn't know what to say, I could not shake the words. I couldn't like I literally had to walk out of there, come over here. And I closed the door. And I said, "Wow, is that what they think about me? -Black, female faculty

The participant was placed in a situation that catalyzed thoughts revolving around how she may be perceived in her department because she identified as a member of the particular group that her colleague had referred to as incompetent. In that instance she felt lumped into a group and judged based on her racial identity. She became less focused on her contributions to the department and more focused on how she was potentially perceived in her department. A case like this could contribute to self-doubt. Similarly, a Black, female administrator expressed:

Someone coming to your office, and assuming that you're the admin, little things like that, you know, or assuming that you're a student. Now, granted, me being sensitive, maybe it's because [I] look young, I look young, you know, could be that [pause] or I don't know [pause] Is it because you don't think that I could have a higher position? You know, because I'm a Black female, things like that, that just make you wonder. Again, maybe it's not that. But I mean, that's the privilege of not being a minority, right. You don't have to worry about if it's that, you know, that's the unfortunate reality of being minority [pause] it does make you wonder [pause] Is it because of that? I mean, did you mistake me for an admin [assistant], because of that, because, you know, you saw a Black female sitting here, or, you know, when you see my supervisor, who's also of color, you know, being surprised and thinking that she's someone else, things like that... But it makes you wonder like, would you do that if she looked differently?... It was interesting working here, because prior I'd never really worked at a majority Hispanic place. So, it is interesting 
working with a vast majority of people who see themselves as not as minorities.

The participant in the above statement expressed being in situations that sent her into a chain of inquiry about why she has certain experiences. She was put in a position to refer back to her racial identity to justify or explain the behaviors of the people she encounters on campus. She also highlights the dynamic of being in a majority-minority environment and how she perceived the dominant groups understanding of self. She stated that the dominant group on campus do not recognize themselves as minorities; this could support the claim that some of the participants made about FIU being a microcosm of the United States. A Black, female faculty also articulated how her experiences on campus influenced how she felt about herself:

An experience like that, it all undermines your confidence. It's undermines everything there because, before that experience, I told you that I grew up in a homogeneous place and you just stand out because you're good... It undermined my confidence because I used to be very- very confident. I used to be. Then all of a sudden, I just figured out, 'hey, you're only as good as they say you are'. It undermined my mindset, my confidence.

Her encounters negatively influenced her confidence. In her case, she has something to compare her concept of self to- coming from a homogenous country where race was not a factor in how she was perceived. She spoke of situations in which colleagues made comments about her ethnicity and how she could be "faking" as a result of stereotypes tied to her country of origin. Her abilities or credentials were not in question in her home country where she was a member of the dominant group; Class played a greater role in how one was perceived versus race, but in her present situation, she understood her race as taking precedence over any other demographic categorization. 
The next participant spoke in how stereotypes influenced her experience and concept of self. The frequency of her negative encounters led her to evaluate why they were happening. The time spent by the participants attempting my make sense of their experiences could be a distractor from their daily activities. The participant stated:

So I felt that I [pause] maybe because I was Haitian or African-American [pause] or- I was viewed as my thought was unimportant or not valid. And I could have felt that way because of the stereotypes, right? That's like [pause] we're all very aware of. It could also be because I look young. I don't know, you know? So, there's a lot of different factors that could... -Black, female administrator

An Afro-Latina, female faculty member made the following mention:

But it can also be insulting at times, because not knowing whether or not they're really interested in the value that I bring or just the fact that I check a box and they have a woman, I'm young, I'm ethnically diverse. So, not knowing whether or not they're really valuing my contributions and if I would be looked at the same if I was a White male, for example. If my contributions would be as valued.

Her self-reflections often lead her to wonder about her presence in her departmentwondering if the contribution of her intersectional identity outweighed her professional contribution. In a case like this, one would understand their identity as being more valuable than their abilities, which could undermine one's efforts.

Self-concept is defined as a person's perception of themselves (Shavelson et al., 1976). Kelley (1973) argues that one's perception of themselves is molded by their experiences with their environment and its corresponding reinforcements. Cooley (1964) argues that one's self-concept is formed as a reflection of the responses and assessments of others in their environment.

Shavelson et al. (1976) explain that "Self-concept is inferred from a person's responses to situations" (p. 411). In the statements presented above, the participants' 
responses to the situations they presented were to self-reflect and essentially question their value and/ or mere existence in their respective workspaces. Some of the participants expressed instances in which their interactions and experiences caused them to develop a sense of self-doubt and insecurity in the workplace. Their encounters, in some cases, altered their psyche and overall sense of self, hence experiencing what I refer to as psyche transformation. This can be linked to a manifestation of external impositions and or an internalization of cultural taxation.

While the participants presented experiences related to cultural taxation, six of the participants also presented instances that served as positive contributions to their experiences, which could in, some cases, work to alleviate the impact of cultural taxation. Some of the participants' feedback regarding these instances include the following:

I feel supported. Maybe it's just that the atmosphere is so good that I kind of feel supported all around regardless of race. So, I have a great boss who supports me, and I have a [pause] group of people who look like me, who are supportive, who listen. -Black, female faculty

In the above case, although the participant felt underrepresented in her department, she spoke of a network of Black women across campus that she identified with racially that she could lean on for support. In addition, she also made mention of how supportive her boss is. Those two entities positively influenced her overall experience at the institution despite her experiences with cultural taxation. Mentorship also served as a positive influence on some of the participants' experiences. One of the Afro-Latina, female faculty members explicitly attributed her success to having "had really supportive mentors." As it relates to promotion, another Black, female faculty articulated the following: 
The second year, I applied [for a higher rank] thanks to the mentoring I got from [Redacted]. I would have been; I was chair of [Redacted] committee. So, in effect, had [pause] I thought [pause] had he turned me down with the second year, I would have been chair of the committee that was going look at my own file. So, you know, she kind of showed me how to maneuver through what the possibilities were at FIU. I was also very lucky in that I've met people who knew what was happening and heard what was happening.

Having a mentor helped the participant progress professionally- Being that she had experienced social isolation, she was removed from how to maneuver the system effectively. Her mentorship contributed to an outcome that differs from some of the participants that expressed feeling trapped because they are not a part of the institutions' inner circle. Her account also highlights how having an "ally" can influence one's career trajectory. Another Black, female faculty member stated:

I have the support of an amazing department chair. And because of her, I'm now able to do things that I never imagined that I could do. I have access to people that I don't believe I would have normally had access to as a faculty member. She gives me the freedom to do what I need to do, despite any pushback I might have from my colleagues. It's only because of her [pause] this idea that she now empowers me to do things and is [pause] it is protecting me from various obstacles and people. She's kind of like carving out this little niche for me to define myself to [pause] to see where I could end up at the university ... I don't even think about, very much, that I'm alone. -Black, female faculty

The notion of having support and its influence on access could presuppose the existence of exclusion and or barriers that may be culturally, ethnically, and or racially grounded. The reference to protection can also be indicative of an opposing force that warrants safeguarding. This is not to say that there aren't other factors that could contribute to these occurrences, but from the participants' understanding, these are perceived to play a role in their experiences. A Black, male administrator expressed: 
God blessed me with good managers. See, my managers love me. Working for FIU and having a manager that was supportive and encouraging was very helpful. To be able to work for them while going through school - and they would work with you as far as tasks, that was helpful.

The aforementioned participant expressed sentiments that were closely linked to socially imposed forms of cultural taxation throughout the interview; however, the support that he received from his managers positively influenced his experience within the institution. A Black, female administrator also made a similar account when asked about things that positively and/ or negatively impact her experience with her colleagues, stating: "You know honestly, I have to say a very good relationship with my director. Whenever I'm really stuck, I go and ask her for guidance."

Again, the intentionality of the exclusion is unknown, and the awareness of the exclusion from the dominant group is also unclear. Social exclusion perceived to be tied to cultural ethnic and/ or racial factors arose among the participants and having a connection to someone, an "insider" was expressed to have a positive influence on one's experience and career trajectory. As presented by the participants, most of their mentors and/ or connections were either White/Non-Hispanic/LatinX or Hispanic/LatinX.

Support was a theme among the participants. Creating a supportive environment is essential to the experiences of underrepresented members of the academy (Alfred, 1999; Gregory, 2002). Although the same participants expressed sentiments of being culturally taxed, they also expressed having support from either supervisors or mentors, which was, overall, conducive to their perception of their experiences. Having adequate support countered the isolation that some of the participants admitted experiencing. 


\section{Chapter Summary}

The study presents the experiences of the underrepresented faculty and administrators as they relate to cultural taxation in an inter-minority setting. The participants provided data based on a series of semi-structured, open-ended questions that were designed to gauge if and how the phenomenon played a role in their professional lives. Throughout the interview process, the participants presented a series of ways that one can experience what I argue is cultural taxation. Their experiences went beyond the explicitly presented forms of cultural taxation, as originally articulated by Amado Padilla. Some of the "six identifiable forms" surfaced but not frequently among the participants. When they did surface, however, they were among faculty, more specifically, Black, female faculty, and faculty that have been in higher education across several years; this is consistent with Amado Padilla's original presentation of the phenomenon and its focus of faculty of color.

Based on Amado Padilla's original definition of cultural taxation: "the obligation to show good citizenship towards the institution by serving its needs for ethnic representation... or to demonstrate knowledge and commitment to a cultural group" and the evolved definition of cultural taxation used for the study: "anything done, or expected of one outside of their job duties because of their cultural, racial, or ethnic differences," I was able to identify alternative ways that underrepresented members of the academy may experience cultural taxation to include: Managing communication and language barriers, coping with students' problematic behaviors, forced social integration, stereotype deconstruction, and differential emotional management. These taxing activities have been classified as such in relation to the energy and or time required or exerted in order to 
accomplish or deal with them as opposed to leaving the environment, which would no longer allow them to serve the institutions diversity needs; thereby, prevailing in spite of the circumstances. As a result of the data provided by the participants, I was able to develop a more refined definition of the phenomenon to capture its multidimensionality, including less visible aspects. Following the collection and analysis of the data, I propose defining cultural taxation as: "The additional physical and/ or mental demands imposed on underrepresented members of the academy irrespective of intentionality or origination."

Three overarching themes and six sub-themes arose during the study. The subthemes were: service, expectations, tokenism, obligations, coping, and integration. The three major overarching themes were in accordance with the origination of cultural taxation (or impositions- external, internal, and social). I felt it was important to distinguish between impositions, or sources, of the phenomenon because, in some instances, the visibility and or tangibility of some might make them easier to mediate and vice versa. Mediation is important because the time and energy spent dealing with these types of cultural taxation can take away from the time spent accomplishing tasks that are conducive to the advancement of their career and in some cases, influence one's mental well-being.

Some of the participants understood their experiences through the lenses of critical agency, while some understood their experiences as an imposed burden. The participants' understanding of their experiences ultimately influenced how they responded to them. Cultural taxation argued as a component of critical agency, tokenism, and/ or oppression was a part of many of the participants' accounts. Critical agency is 
being understood as one's act of critically assessing and making conscious efforts to reject existing norms (Poveda \& Robert, 2017); its link to cultural taxation is tied to ones' efforts in the workplace and its recognition as something that requires additional time and efforts negated from their job duties, which are rooted in their demographic difference. In some instances, one can build critical agency into their work; however, this is not always the case.

Tokenism is understood as a symbolic attempt to serve the institutions' need for ethnic representation, and one's mere presence, as an institution's attempt to satisfy diversity requirements, can be argued as a tax paid by a minority. Oppression is being linked to cultural taxation as it relates to a series of elements; The first being its understanding as a form of invisible labor, second, as it relates to one's demographic difference and the actions catalyzed by ones difference- specifically referencing Young's (2013) faces of oppression, and finally, the intentionality of the institution and its constituents can which can also influence the phenomenon's classification as oppressive.

Prior literature emphasized cultural taxation as an entity imposed by the institution which ignored other forces that, too, can induce cultural taxation. The absence of data supporting alternative applications and understanding of the phenomenon can limit exposure of more discrete forces that influence the experiences of underrepresented members of the academy. Typically, faculty members experienced externally imposed forms of cultural taxation across the board, while both faculty and administrators shared experiences that related to externally, internally, and socially imposed forms of cultural taxation. In addition to the newly surfaced forms and understandings of the phenomenon is the newly emerged consequence of cultural taxation: psyche transformation. Please 
refer to appendix B for a visual mapping of the phenomenon, its impositions, and how it can be experienced. I provide this to present a compressed version of the information provided in this chapter.

Overall, the data provided by the participants confirm prior literature regarding cultural taxation, namely in the existence of pressures imposed on underrepresented members of the academy. Where it extends the literature is in its application to an alternative demographic, its inclusion of administrators, and its investigation into more discrete forces that induce physical and mental demands. 


\section{CHAPTER VI}

\section{FINDINGS AND IMPLICATIONS}

This chapter recaps the purpose and design of this qualitative interview study. It then presents the study's findings as well as an exploration of cultural taxation using an economic framework; this was inspired by the understanding of cultural taxation as a form of invisible labor. Furthermore, this chapter breaks down and organizes cultural taxation into a series of categories/types. It then proceeds to present the implications and recommendations for practice and research and then concludes with my final thoughts and remarks.

\section{Summary of the Study}

The purpose of the study was to expand the application of the notion of cultural taxation outside of its traditional application to faculty of color in predominately White settings. The study sought to explore a more extensive application of cultural taxation to include faculty and university administrators at Florida International University (FIU), an MSI/HSI with a workforce demographic composition that consists of, predominately, members that would classify as minorities. While exploring the data presented by the participants, I was also able to expand the understanding of the phenomenon to encompass the source of the pressures underrepresented members of the academy may encounter- which were what I presented in the previous chapter as external, internal, and social impositions. Furthermore, I presented the phenomenon in accordance with alternative understandings to include being understood as: an obligation, a component or act of critical agency, a form of tokenism, and a form of oppression. Also, I formulated a definition that I understood as more appropriate considering my findings. 
This qualitative study utilized interviews to probe into the experiences and perceptions of cultural taxation among underrepresented faculty and administrators at FIU. The study aimed to answer the following questions: 1 . To what extent do faculty and administrators experience cultural taxation at Florida International University, Modesto Maidique campus? 2. How do the experiences of faculty and administrators compare with regard to cultural taxation? I asked the participants semi-structured, openended questions framed around the published components of cultural taxation in addition to broader questions designed to capture any component that may not have been addressed to allow participants to construct responses beyond the context of my questions.

Based on the accounts of 15 underrepresented faculty and administrators, I extended the understanding of cultural taxation in four ways. First, in its application to administrators in higher education as the understanding of cultural taxation has traditionally been aligned with faculty of color. Second, in the phenomenon's application to an institution that is not classified as a PWI. Third, I was able to present additional ways that the phenomenon can be experienced that were not previously identified in literature. Finally, I was also able to lean away from a more concrete, inflexible understanding of cultural taxation to a more fluid understanding that allows the phenomenon be understood beyond being a series of allocated or requested tasks, but also link it to a series of circumstances that attract certain behaviors and actions from underrepresented members of the academy that may lack the visibility to attract inquiry, i.e., what I present as internal and social impositions of the phenomenon. 
The participants generally experienced some form of cultural taxation or another; some experienced multiple forms of cultural taxation simultaneously, while some participants were "textbook cases" in relation to the phenomenon. Though the participant's experiences generally related to the previously published forms of cultural taxation, upon analysis of their accounts, I was able to identify and present other ways the phenomenon can be experienced. The major themes that arose and transcended into the development of alternative understandings of cultural taxation are service, expectations, obligations, coping, and integration, which could be categorized under one form of imposition or another.

Moving forward, I will illustrate how different forms of cultural taxation are imposed and then present the research finding in accordance with the original research questions. I will address the question regarding the extent to which faculty and administrators experience cultural taxation generally and then specifically by focusing on faculty and then administrators.

The following chart presents the impositions of cultural taxation and some of the ways it can be experienced.

Externally Imposed Forms of Cultural Taxation

- Being called upon to serve as the "expert" on diversity matters

- Being called upon to educate members of a majority group about diversity

- Serving on affirmative action committees without any observable changes

- Serving as the liaison between the institution and the ethnic community even though one may not agree with the institutions' policies

- Being called upon to serve as a translator or interpreter when the institution is presented with nonEnglish speakers

- Mentoring

- Differential Legitimacy

- Irregular Expectations 
Internally Imposed Forms of Cultural Taxation

- $\quad$ Taking responsibility for settling problems regarding sociocultural differences among the members of the institution

- Mentoring

- Coping with colleagues' problematic behavior

- Coping with students' problematic behaviors

- Stereotype deconstruction

- Differential emotional management

- Critical Agency Socially Imposed Forms of Cultural Taxation

- Acculturation

- Managing communication and language barriers

- Forced social integration

The chart illustrates the ways that participants in the study experience cultural taxation, in addition to Amado Padilla's original six identifiable forms and other extended forms as presented by other scholars.

\section{Extent Faculty and Administrators Experience Cultural Taxation}

The findings in the study present that cultural taxation is a phenomenon that is not exclusive to faculty of color in predominantly White spaces; it can exist among administrators as well. It also presents that the environments in which cultural taxation can exist are not exclusive to predominantly White spaces, but instead, any space where there is over- and under-representation. Overall, the experiences of both faculty and administrators in the study relate to cultural taxation to a certain extent; the extent was dependent on their respective spaces on campus, the quality of their relationships with their colleagues, their relation to the dominant group, and in some cases in which they fell on the institutional hierarchy; This was consistent across different races and ethnicities. All fifteen participants made references to being culturally taxed in some way, 
shape, or form. The extent, as well as the ways faculty and administrators experienced cultural taxation, did, however, vary among the participants.

I gauge "extent" based on three elements: occurrence, frequency, and the articulation of signs indicative of the impact of cultural taxation as presented by the participants as it relates to career progress, job satisfaction, and burnout (Mahoney et al., 2008; Hirshfield \& Joseph, 2011; Pololi et al., 2010; Hassouneh, et. al., 2014; Mahoney, et al., 2008; Kolade, 2016; Trower, 2003; Blackburn \& Lawrence, 1995). I base occurrence on an understanding that cultural taxation is relevant and influential whether an individual experiences one, multiple, or all forms of cultural taxation; Therefore, if any form of cultural taxation, single or multiple, occurred as a part of a participants experience, I argue that it counts as an influential component of their experience. I base frequency on how often specific forms of cultural taxation surfaced. Moving forward, I will present the findings of the second research question that serves to compare the experiences of faculty to administrators.

Underrepresented Minority Faculty, Administrator and Cultural Taxation at FIU

I interviewed eight faculty members: four Black, two White, and two Multi-race. They experienced cultural taxation in several ways. In reference to external impositions, six faculty members reported having culturally taxing experiences related to expectations tied to their cultural, racial, or ethnic association — six reported experiences related to service and three shared experiences related to tokenism. In relation to internally imposed forms of cultural taxation, seven faculty members shared experiences related to coping while all eight expressed having had experience some form or another of cultural taxation 
tied to feelings of obligation. Social impositions of cultural taxation arose as three faculty referenced integration as a part of their experience.

I interviewed seven administrators: six Black and one White. As their experiences relate to external impositions of cultural taxation, they all reported having had encountered expectations linked to their racial, cultural, or ethnic difference. Two administrators shared experiences associated with service and one reported experiences related to tokenism. As their experiences related to internal impositions of the phenomenon, three of the participants shared experiences involving coping with the situations they encounter, and three presented experiences tied to feelings of obligation. Five of the six participants shared experiences that fall under socially imposed forms of cultural taxation as they shared instances related to efforts to integrate themselves into the campus community socially.

The results of the study show that both faculty and administrators experienced some form of cultural taxation or another. Instances related to expectations were common among both faculty and administrators, while instances related to service were presented by faculty to a greater extent. This finding supports the idea that underrepresented faculty "are committed to service to the larger community and are intent on making a difference in society" (Laden \& Hagedorn, 2000, 64), and view "service [activities] as a way to give back... and effect positive change" (Stanley, 2006, p. 704). This is not to say that administrators do not share similar sentiments, but may not feel they have the platform or resources to induce change.

Coping was more common among faculty than administrators. Interestingly enough, coping was less common among the administrators in the study, and two have 
departed from FIU since the interview; this could be indicative of how coping can influence persistence. Given the data provided by the two participants that sought employment elsewhere, their departure aligns with studies that address burnout; although Maslach and Schaufeli (2001) argue against the problematizing of "individual-centered solutions" which would include ones' ability to cope in certain situations; they instead, place greater responsibility on the "situational and organizational factors" that influence burnout. Maslach and Goldberg (1998) explain that there is an underlying assumption that it is more cost efficient to have an employee replaced than it is to change an organization.

Faculty in the study also carried a greater sense of obligation than the administrators. Evans (2007) states that leaders in the academy "have a social and moral obligation to foster equitable... practices" (p. 250). Finally, as it relates to integrating oneself into the campus community, administrators expressed this more often than faculty. This is consistent with studies that address social-exchange theory which imply that people form relationships for their own benefit. The benefits in this case are resources; social resources which include social goods like "approval [and] esteem" (Blau, 1664, p.1233). Faculty in the study may have not felt the need to acquire further approval or esteem unlike their administrative counterparts.

External impositions were more frequently expressed by faculty, as were internal impositions, but social impositions were more frequently expressed by administrators; this could be associated to ranking which could, in some cases, demand a degree of tolerance from the dominant group, or it could be linked to status one's conceptualization 
of self which could make one feel as though they do not, or should not have to strive to be socially accepted.

The faculty participants in the study generally dealt with cultural taxation to a greater extent. This conclusion is based on the fact that all three impositions regularly surfaced among them; it shows that they not only faced externally imposed forms of cultural taxation, but they also faced internally and socially imposed forms of cultural taxation. The extent, however, does not presuppose the invisibility of the experiences of administrators which further highlights the importance of the study. Generally, underrepresented administrative participants expressed experiences that aligned with the externally and socially imposed forms of the phenomenon.

Some of the participants' experiences are consistent with studies that explore minority on minority prejudice and discrimination (Chen, 2010; Harbi, 2016). The overall social approval of behaviors by traditionally dominant groups can lend themselves to the adoption of such behaviors by other minorities in dominant rankings. Hispanics are the dominant minority in the United States (Makert, 2010). Miami, in particular, is a Hispanic-dominant city, making about 70\% of Miami-Dade County’s population according to the U.S. Census Bureau, and this is reflected in FIU's demographic makeup, which created an environment that is conducive to cultural taxation.

\section{The Economics of the Cultural Tax}

In this section I present cultural taxation through the lenses of actions "paid" out by those who experience it. I analyze the understanding by exploring who or which entities benefit from these cultural taxes, and by the "currency," or as I present them: 
"output "categories." I use this approach to highlight the "cost" and "beneficiaries" of the phenomenon.

The Allocation of the Cultural Tax: For the Culture and For the Institution

In understanding cultural taxation as services "paid" out to the institution, I critique the participants shared experiences through an economic/allocation framework, which was inspired by Karl Marx's evaluation of capitalism. I took this approach to flesh out how and who benefits from the services fulfilled by underrepresented faculty and administrators. A framework is essentially a way of seeing. Exploring cultural taxation through an economic lens is intended to provide additional perspective on social experiences. The study revealed a series of motivational factors that contributed to the experiences of the participants. Some of the culturally taxing activities the participants shared were imposed by the institution, while some were self-imposed for a variety of reasons, which were often centered on the betterment of their respective underrepresented groups or underrepresented groups they had a commitment to.

The interview protocol inquired about internal and external impositions of cultural taxation each participant faced throughout their professional career at FIU. The findings presented that the external impositions were often meant to serve the needs of the institution, while the self-impositions were often done in an effort to advance one's cultural or racial groups' standing or degree of representation within the institution in hopes of it serving as a domino effect for society as a whole. These two allocations are not mutually exclusive; they can intersect with one another as it relates to outcomes. This led me to reflect on who or what is the ultimate beneficiary of the allocations: the institution or "the people?" 
Furthermore, is the concept of invisible labor. In this instance, cultural taxation is an, often, unmeasurable workload that lacks compensation for the individuals completing the tasks at hand. While the labor is "invisible," it is still felt by institutions of higher education, thereby making it a tax allocated directly to the institution; this provides alternative means for understanding some forms of cultural taxation, particularly those that directly impact the institution, from invisible labor to free labor.

The evolution of the presence of underrepresented professionals in higher education can be viewed as a marathon. The allocation to the university can be seen as steadily progressive. In contrast, the allocation to "the culture" (or people) can be seen as somewhat stagnant because while it progresses the institution and the members of the underrepresented communities, the faculty and administrators in higher education experiencing cultural taxation often carry the cost by way of burnout, over-commitment, isolation, career progress impediments, and psyche transformation.

Regardless of the intentions or allocations of these activities, some forms of cultural taxation can be viewed as a form of control or discipline, be they internally or externally imposed. Foucault (1977) argues that:

Discipline... arranges a positive economy; it poses a principle of a theoretical ever-growing use of time...It is a question of extracting; from time, ever more available moments and, from each moment, ever more useful forces (p. 153).

Focusing on the "use of time," the institution can be seen as maximizing the time of the underrepresented faculty and administrators by utilizing them in ways that may be overextensive to meet specific needs or standards, thereby promoting a "positive economy" for the institution. Additionally, some underrepresented faculty and administrators could 
also be seen as maximizing their time and presence by intentionally participating in culturally taxing activities. Maximized use of time is obtained from the underrepresented faculty and administrators at hand to compensate for their shortage within the institution, which also has a connection to tokenism.

\section{Cultural Taxation as Workplace Oppression}

The notion of cultural taxation's understanding of being a form of invisible labor that oftentimes goes without compensation links itself to Young's (2013) understanding of exploitation as a form of oppression. In following Karl Marx's evaluation of capitalism as a system that creates "haves" and "have-nots," one might critique the relationship between the institution and underrepresented faculty and administrators as a "haves and the have-nots" dynamic; The institution as the "haves" as it benefits from the invisible labor, and the underrepresented faculty and administrators as potential "have-nots" as they are often not compensated for their acts of service while also, at times, being placed in somewhat of a deficit position in relation to promotion and tenure in conjunction with their additional workloads and efforts. This system perpetuates a hierarchy that could aid in the maintenance and progression of the institution while simultaneously serving as a setback for the underrepresented faculty and administrators.

Young (2013) explains that most often, people are marginalized based on race; this theory is consistent with the experiences of the participants of the study as one of my Afro-Cuban participants experiences aligned, mostly, with her non-Hispanic counterparts presenting that her race outweighed their cultural connection. Powerlessness, too, was a part of the participants' experiences. Cultural imperialism, as another face of oppression, involves taking the culture of the dominant group and establishing it as the norm. The 
dominant group that holds power in a particular space controls how individuals in that particular space interpret and communicate. Young refers to "society." For this analysis, FIU is the "society" or space. The ways and beliefs of the dominant group are widely disseminated, and individuals that are impacted by this form of oppression are often plagued by stereotypes and experience a sense of invisibility.

In reference to violence, the institution and/or the dominant group within the institution possess the power to impose or provoke cultural taxation on underrepresented members of the academy; this imposition or provocation could, in some instances, be understood as a form of "violence" by the institution. The institution serves as the workplace for faculty and staff; therefore, cultural taxation could be understood as workplace violence. I use the definition by Bowie (2002) to define workplace violence as "A perceived or actual verbal, emotional threat or physical attack on an individual's person or property by another individual, group or organization while undertaking workrelated duties." Therefore, underrepresented members of the academy who experience cultural taxation in addition to their work-related duties could be understood as being subjected to a form of violence.

Categories/Types of Cultural Tax

What I managed to do is to further expound on the understanding of cultural taxation in accordance to how it is experienced, who can experience it, and the multiple forces that serve as the source of the phenomenon. Following the analysis of the data, four output categories were developed. While the different forms of cultural taxation can be divided into subcategories, some can fall into multiple subcategories. The forms of cultural taxation explored in the study were broken down into the following categories: 
Emotional Cultural Taxation, Psychological Cultural Taxation, Financial Cultural Taxation, and Time Cultural Taxation.

Emotional (feelings), Psychological, Financial and Allotment Cultural Tax

In the realm of emotional cultural taxation, the phenomenon serves as a tax on one's emotional well-being. The tasks or encounters that take place take an emotional toll on underrepresented. Cultural taxation could be understood as a form of emotional violence. The imposition or provocation of actions and scenarios by dominant groups that evoke negative emotions from underrepresented groups would fall under this category of cultural taxation. I developed this understanding from some of the participants' feedback regarding how certain situations made them feel. If this aspect is also linked to burnout, it can support claims that "burnout...precipitates negative effects in terms of mental health, such as anxiety and depression" (Maslach \& Schaufeli, 2001, p. 406).

In this context of psychological cultural taxation, the phenomenon acts as a tax on one's psyche. This type of cultural taxation causes one to question themselves, their fit, abilities, and value. These types of cultural taxations lead to self-doubt, morale reduction, and insecurity in the workplace. I developed this understanding from some of the participants' accounts regarding self-reflection and how they assessed their value within their respective departments. In these cases, participants understood that their value was attributed to their cultural, racial, or ethnic identity instead of their abilities, which was linked to how they conceptualized themselves and existence in the spaces they occupied. Stanley (2006) states that when underrepresented members of the academy have certain encounters on campus "it can take a toll on their psyche, often forcing them to question whether it was due to race or not" (p. 711). 
Financial cultural taxation relates to any form of cultural taxation that has any financial implication. As a form of invisible labor, or exploitation, individuals that participate in culturally taxing activities often do not receive compensation for them. This kind of cultural taxation can also be applied to the long-term as it relates to issues concerning promotion and tenure (Blackburn \& Lawrence, 1995; Griffin et al., 2011; Rodriguez et al., 2015; Trower, 2008; Wijesingha \& Ramos, 2017). I developed this category from prior literature that addresses "invisible labor" and a correlation between cultural taxation and promotion and tenure which both have financial implications.

Allotment cultural taxation is a tax on one's time and is perhaps the most universally applied form of cultural taxation. This relates to any form of cultural taxation that negates time away from prescribed work duties. Here, individuals are burdened with more responsibilities outside of their professional work while being held to the same standard as their majority counterparts. This output can serve as the umbrella for the other outputs as they all impede on one's available time.

Something worth investigating is the intentionality of the imposition of cultural taxation. Are these purposeful acts or acts done unwittingly? The chart on the next page provides a visual illustration of how cultural taxation can be imposed and its allocations; it is not representative of a model but intended to paint a picture of the inputs and output of cultural taxation. 


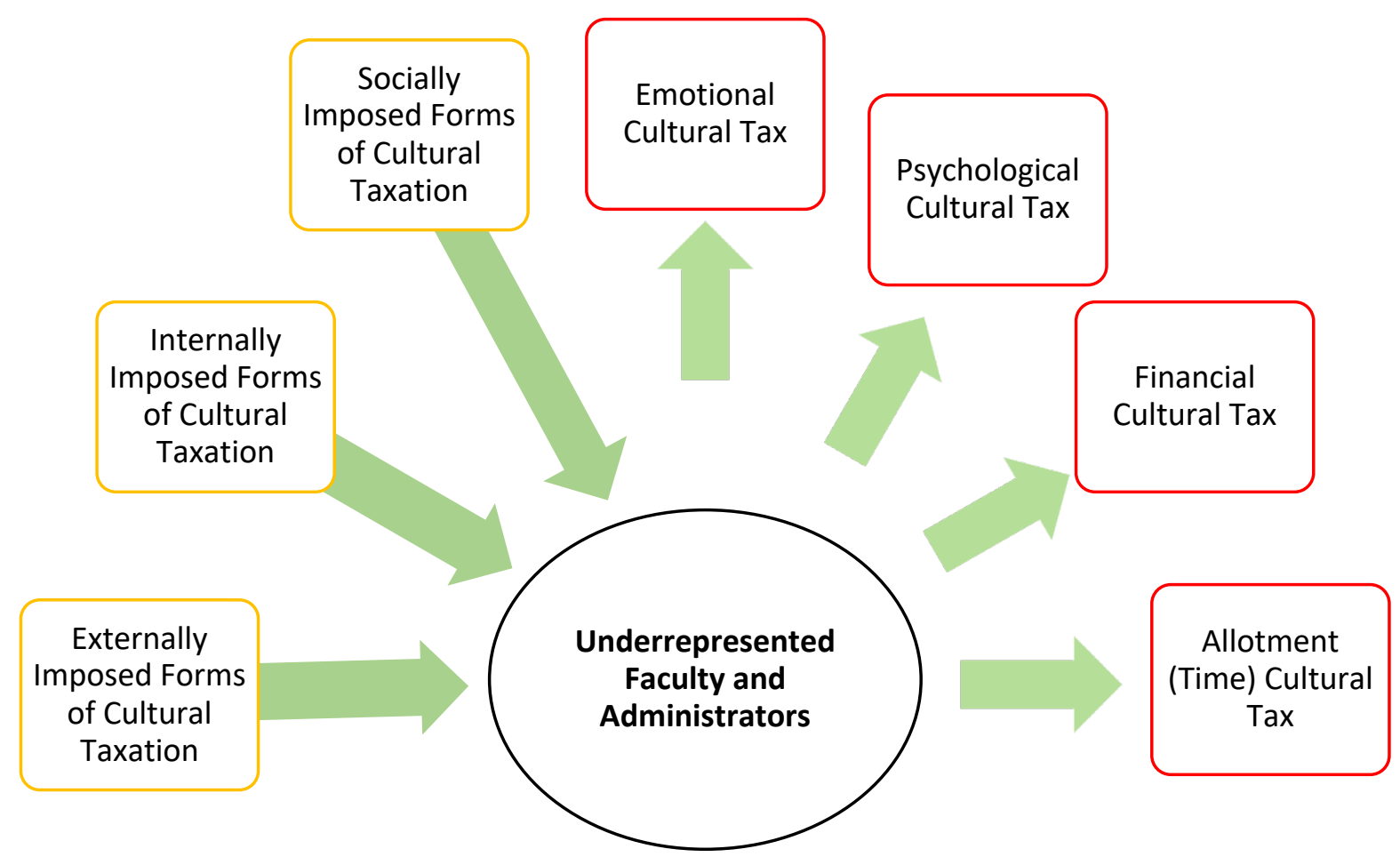

The chart is presented to compress the text in this dissertation to illustrate the several forces that underrepresented faculty and administrators can encounter in the academy as well as the several costs associated with the impositions. In acknowledgment of the inputs and outputs, I present a series of implications and recommendations for practice in the following section.

\section{Implications and Recommendations for Practice}

There are a series of implications for practice that surfaced during the study. At the institutional level, upper leadership could improve recruitment and hiring practices that support greater diversity in higher administrative roles; This could have a trickledown effect on the overall experience of the workforce in its entirety by providing exposure to different demographics thereby potentially breaking down stereotypes 
(Hassouneh et al., 2014), providing greater diversity in decision-making, and reducing a sense of isolation among underrepresented groups. Many participants mentioned a noticeable absence of underrepresented members in upper-level leadership roles, how they felt as though they did not have anyone to reach out to for mentorship, and how they did not feel as though they had the opportunity to advance to upper-level administrative roles within the institution.

Current policies can also undergo review to explore how they might perpetuate inequities among underrepresented faculty (in particular) and administrators. Some policies, as they relate to tenure and promotion, have the tendency to work against underrepresented faculty, especially when presented with disproportionate service loads (Blackburn \& Lawrence, 1995; Trower, 2003). This area of inquiry, however, does not take into account any psychological drains that may accompany one's experience as it relates to the time and energy that are necessary to cope and prevail, which, too, presents an area of exploration that is outside of my expertise.

At FIU, particularly, I would like to advocate for the incorporation of regular, mandatory diversity, inclusion, and sensitivity training that may work to alleviate some of the negative experiences that underrepresented members of the community express experiencing in their work environments. "Coping with colleagues' problematic behaviors" was one of the most referenced forms of cultural taxation in the study. Without inducement, many of the participants (including Afro-Cuban and Afro-LatinX) referenced the Cuban population (in particular) in Miami and FIU, and their influence on their experience within the institution. The most common issues revolved around being overcome by what felt like a non-inclusive culture, experiencing exclusion by way of 
language (i.e., speaking Spanish in work meetings and environments that are intended for everyone even though everyone didn't speak Spanish), and dealing with what could be classified as racist remarks and behaviors. This is not to generalize any specific population but to bring to light some of the experiences some of the participants shared. Exposure to different cultures can be a beautiful thing, and it is conducive to higher education to allow it to permeate the environment as we learn from one another, appreciate and celebrate our differences. However, equally, conducive is the practice of acceptance over tolerance as it relates to members of different racial and cultural groups. This component (Cuban culture) was so saturated within the participants' responses that it almost reshaped the dynamic of the interviews throughout the study. I would find myself having to redirect some interviews back to cultural taxation as my questions regarding coping with colleagues' problematic behaviors, acculturation, isolation, and career progress would ignite some mention of FIUs Cuban community.

Coping with colleagues' problematic behaviors was a concept explored by Hirshfield and Joseph (2011) and was a foundational component of this studies interview protocol, of which led to the abstraction of the reoccurring theme: Coping with students' problematic behaviors. Again, in the study, I defined problematic behaviors as any actions or statements that intentionally or unintentionally has an adverse mental or physical effect on the receiving party. Stanly (2006) addressed some of the challenges underrepresented members of the academy may face, to include dealing with students who question and undermined their intelligence in academic spaces, which supports the data provided by the participants in the study. The prevalence of this topic during the interviews suggest that there should be an implementation of some accountability for 
students who perpetuate toxic behaviors towards underrepresented faculty and administrators; This is particularly important because students are the future, and will one day, too, be working in environments that may mirror the demographics that they are educated in, and as leaders in higher education we should aim to avoid the reproduction of the behaviors addressed in the study. Additionally, as Brayboy (2013) suggests, it is essential to incorporate diversity-related issues into mainstream courses and that "[Relegating] the issues to special topics not only ghettorizes the material and the course but also sends a clear message to students that issues of diversity are tangential” (p. 83).

Finally, because some of the participants emphasized their gratitude for the support they receive from their bosses and/or mentors, and the impact that support has had on their professional development despite their experiences with cultural taxation, it may be valuable to provide resources for leadership on how to support underrepresented members of the academy.

\section{Recommendations for Future Research}

The findings in the study led me to want to explore the phenomenon from a series of angles. The first approach that surfaced as I was evaluating the dynamics of cultural taxation and critical agency was how the phenomenon might impact underrepresented faculty and administrators who work at institutions where their particular minority group predominates. In a case like that, some components of cultural taxation have the potential to become magnified because those institutions serve an increased number of minority students who may have unique needs associated with their minority status, potentially lower socioeconomic status, first-generation college attendee status, and lower academic preparation due to the likelihood of having had attended poorly funded k-12 schools (Niu 
\& Tienda, 2011; Falcon, 2015; Brock, 2010; Harmon, 2012). In such a case, a study would almost directly mirror this one; however, the participants would be the exact individuals that were excluded from my selection criteria.

Future studies could also explore cultural taxation at other MSIs such as Historically Black colleges and universities, Tribal colleges or universities, Alaska Native or Native Hawaiian serving-serving institutions, predominately Black institutions, Asian American and Native American Pacific Islander-serving institutions, and Native American-serving nontribal institutions. These types of institutions may be seen as the "Ivory Tower" of higher education institution types; however, some of the concepts explored in the study might provide valuable insight.

Another angle would be to explore cultural taxation, by exploring the extent to which underrepresented students experience cultural taxation. I believe this, too, has the potential to shed light on the dynamics of cultural taxation and how it may impact the minority students' collegiate experience. Furthermore, looking further into psyche transformation, it may be interesting to conduct a longitudinal study that investigates minority faculty and administrators' sense of self and confidence in higher education over the years.

Another recommendation is to further investigate the less tangible/visible forms of cultural taxation as I understand them; this would focus heavily on internally and socially imposed forms of the phenomena. I presented them as a new understanding of the phenomenon, and I think they are worth investigating because they are more discreet than other forms of cultural taxation, which could make them more difficult to mediate. 
I believe it would also be beneficial to explore perceptions and conceptualizations of diversity. This is important considering how often colleges and universities emphasize diversity and incorporate them into their missions and dialogues; however, what does diversity actually mean to the institution and its members? Understanding how people understand diversity could aid in understanding individual behaviors and institutional practices. Conflicting understandings of diversity are not conducive to progressive action. In addition, one of my participants had a physical disability, and the information shared sparked my interest. Much of the participants' experience with cultural taxation paralleled with her experience as an individual with a physical disability. Throughout the study of cultural taxation, the notion of difference weighed in heavily. The participants experience with cultural taxation were a result of their racial, ethnic, and/or cultural difference. Similarly, the participants' physical difference also attracted situations comparable to cultural taxation. The participant had a unique experience based on the intersectionality of her underrepresented ethnic and racial identity, as well as her disability status. I would recommend conducting a study that explores cases like this one and how individuals with physical disabilities might face additional external, internal and/ or social pressures in the academy.

My final recommendation for further research would be to explore cultural taxation from the dominate groups' perspective and to study their practice of imposing it on their counterparts. Too often, the experiences of underrepresented members of the academy are explored through their lenses but rarely through the lenses of the "perpetrators." I believe this would provide better insight on how to mediate some of the experiences related to the phenomenon. This, too could aid in understanding the 
intentionality of some of the culturally taxing activates and the awareness or lack thereof awareness of how certain behaviors, requests and or demands influence the experiences of their underrepresented counterparts.

\section{Final Thoughts and Concluding Remarks}

The notion of cultural taxation has evolved since its original understanding, presented by Amado Padilla (1994). It has transitioned from a being used to describe a phenomenon experienced by faculty of color to being used to describe the experience of faculty and administrators in collegiate spaces where they are underrepresented.

I found this process to be both therapeutic and violent at times. In some cases, it was refreshing to see that I was not alone; that others shared similar experiences as me. In some cases, however, some of the accounts from my participant began to make me question my own place and purpose within the institution and higher education as a

whole. I presume I fell into a phase of psyche transformation, ultimately paying a cultural tax in an effort to conduct this research.

Nevertheless, I hope the study illuminates the sometimes-hidden experiences of underrepresented members in spaces that may be assumed to be fair and just because of their racial and cultural demographics. Hopefully, it aids in helping senior-level administrators, and faculty acknowledge and understand some of the issues that underrepresented members of the academy may encounter in environments that are often understood and referred to as diverse, and "untarnished by the ugly inequalities that mar the "outside world" (Stockdill \& Danico, 2012, p.1). 


\section{References}

Ahmed, S. (2007). A phenomenology of Whiteness. Feminist theory, 8(2), 149-168.

Alfred, M. (1999). Expanding theories of career development: Adding the voices of African American women in the White academy. Adult Education Quarterly, 51, $108-127$.

Alleyne, A. (2005). Invisible injuries and silent witnesses: The shadow of racial oppression in workplace contexts. Psychodynamic Practice, 11(3), 283-299.

Ansari, S., Panhwar, A., \& Shah, A.. (2017). Retrieved from http://http://search.ebscohost.com/login.aspx?direct=true $\& \mathrm{db}=$ hus \&AN=1241400 $68 \&$ site $=$ eds-live

Austin, A. E., ERIC Clearinghouse on Higher Education, W. D., \& American Association of Univ. Administrators, W. D. (1984). The Work Experience of University and College Administrators. Administrator's Update, 6(1). Retrieved from http://http://search.ebscohost.com/login.aspx?direct=true \&db=eric\&AN=ED2596 90\&site $=$ eds-live

Baez, B. (2000). Race-related service and faculty of color: Conceptualizing critical agency in academe. Higher Education, 39, 363-391.

Barnett, R. (2010). Being a university. Routledge. Chicago

Bassnett, S. (2005). The importance of professional university administration: a perspective from a senior university manager. Perspectives: Policy \& Practice in Higher Education, 9(4), 98-102. https://doi.org/10.1080/13603100500357274

Bell, V. (1999). Performativity and belonging: An introduction.

Blackburn, R. T., \& Lawrence, J. H. (1995). Faculty at work: Motivation, expectation, satisfaction. Baltimore, MD, US: Johns Hopkins University Press.

Blake, D. (2018). Motivations and Paths to Becoming Faculty at Minority Serving Institutions. Education Sciences, Vol 8, Iss 1, p 30 (2018), (1), 30. https://doi.org/10.3390/educsci8010030

Blau, P. M. (1964). Exchange and power in social life. New York: Wiley. Bluedorn, A. C. (1982). A unified model of turnover from organizations. Human Relations, 35, $135-153$. 
Boddy, C. R. (2016). Sample size for qualitative research. Qualitative Market Research: An International Journal, 19(4), 426-432. https://doi org.ezproxy.fiu.edu/10.1108/QMR-06-2016-0053

Bowie, V. (2002). Workplace violence: A second look. In Crime Prevention Conference. University of Western Sydney.

Brayboy, J. McKinley, B. (2003). The Implementation of Diversity in Predominantly White Colleges and Universities. Journal of Black Studies, 34(1), 72-86. Retrieved from http://www.jstor.org/stable/3180858

Brislin, R. (2008) Working with Cultural Differences: Dealing Effectively with Diversity in the Workplace, Praeger Publishers, Westport, CT, USA, ISBN-13: 9780313352829 .

Brock, T. (2010). Young adults and higher education: barriers and breakthroughs to success. The Future of Children, 20(1), 109-132.

Brookfield, S. (2019). Why we need critical theory to survive adult life. The Higher Educations Program's Educational Inquiry Colloquium Series. Lecture. Florida International University.

Brubacher, J., \& Rudy, W. (1997). Higher education in transition: A history of American colleges and universities (4 ed.). New Brunswick: Transaction Publishers.

Canton, C. E., Cintrón, J., \& Jacobs, L. (2002). The politics of survival in academia: Narratives of inequity, resilience, and success. Lanham, Md: Rowman \& Littlefield.

Chang A., Martinez, M., \& Welton A.. (2017) Assistant professors of color confront the inequitable terrain of academia: a community cultural wealth perspective, Race Ethnicity and Education, 20:5, 696-710, DOI: 10.1080/13613324.2016.1150826

Chen, C. (2010). Minority on minority discrimination: Impact of majority social norm Perception. Carnegie Mellon University.

Comas-Diaz, L. (1996). Latinegra: Mental health issues of African Latinas. In M. Root (Ed.), The multiracial experience (p. 167-190). Thousand Oaks, CA: Sage

Collier, V. P. (1987). Age and rate of acquisition of second language for academic purposes.TESOL quarterly, 21(4), 617-641.

Connelly, L.M. ( 2008). Pilot studies. Medsurg Nursing, 17, 411-412 
Cooley, C. H. (1964). Human nature and the social order. 1902. New York: Charles Scribner's Sons.

Creswell, J. W. (2003). Research design: Qualitative, quantitative, and mixed methods approaches (2nd ed). Thousand Oaks, CA: Sage Publications.

Creswell, J. W. (2005). Educational research: Planning, conducting, and evaluating quantitative and qualitative research (2nd ed.). Upper Saddle River, NJ: Pearson

Creswell, J. W., \& Poth, C. N. (2018). Qualitative inquiry \& research design: Choosing among five approaches.

De Kadt, E. (1993). Language, power, and emancipation in South Africa. World Englishes, 12(2), 157-168.

Dulay, H., \& Burt, M. (1978). From research to method in bilingual education. In J.E. Alatis (Ed.), Georgetown University Round Table on Languages and Linguistics (p. 551-575). Washington, DC: Georgetown University Press.

Elliott, V. F. (2018). Thinking about the coding process in qualitative data analysis. Qualitative Report, 23(11).

Elmore, P. B., Green, J. L., \& Camilli, G. (2006). Handbook of complementary methods in education research. Mahwah, N.J. : Lawrence Erlbaum Associates ; Washington, D.C. : Published for the American Educational Research Association.

Evans, A. E. (2007). Horton, Highlander, and leadership education: Lessons for preparing educational leaders for social justice, Journal of School Leadership, 17, 250-275.

Falcon, L. (2015). Breaking down barriers: First-generation college students and college success. Innovation Showcase, 10(6).

Feagin, J. (2013). Systemic racism: A theory of oppression. Routledge.

Fernandez, C. (1996). Government classification of multiracial/multiethnic people. In M. Root (Ed.), The multiracial experience (pp. 15-36). Thousand Oaks, CA: Sage.

Foucault, M. (1977/2012). Discipline and punish: The birth of the prison. Vintage.

Foucault, M. (1980). Power/knowledge: Selected interviews and other writings, 19721977. Vintage.

Freire, P. (1970). Cultural Freedom in Latin America. Human rights and the liberation of man in the Americas, 162-179. 
Freire, P. (2005). Pedagogy of the oppressed. The Continuum International Publishing Group.

Geertz, C. (2008). Thick description: Toward an interpretive theory of culture. In The cultural geography reader (pp. 41-51). Routledge.

Grant, B. J. (2017). Tokenism. The SAGE Encyclopedia of Political Behavior.

Green, K. A., López, M., Wysocki, A., \& Kepner, K. (2002). Diversity in the workplace: Benefits, challenges, and the required managerial tools. University of Florida, 1(4), 1-3. Chicago

Gregory, S. (2002). Black faculty women in the academy: History, status, \& future. Journal of Negro Education, 70, 124-138.

Griffin, K. A., Bennett, J. C., \& Harris, J. (2011). Analyzing gender differences in black faculty marginalization through a sequential mixed-methods design. New Directions for Institutional Research, 2011(151), 45-61.

Grissom, A. R. 1. shoresml@miamioh. ed. (2018). Workplace Diversity and Inclusion. Reference \& User Services Quarterly, 57(4), 242-247. https://doi.org/10.5860/rusq.57.4.6700

Guba, E., \& Lincoln, Y. (2011). Paradigmatic controversies, contradictions and emerging confluences. (3rd ed.). Sage.

Guzmán, A. (2016). Founding a Historically Latino/Caribbean-serving Institution: An Archival Research Study on Florida International University. LSU Doctoral Dissertations. 2706. https://digitalcommons.lsu.edu/gradschool_dissertations/2706

Harbi, N. (2016). Inter-minority Racial Prejudice and Anti-White Bias: an Underestimated Phenomenon. Revue EXPRESSIONS n.

Herring, C. (2009). Does Diversity Pay?: Race, Gender, and the Business Case for Diversity. American Sociological Review, 74(2), 208-224. https://doi.org/10.1177/000312240907400203

Harmon, N. (2012). The role of minority-serving institutions in national college completion goals. Washington, DC: Institute for Higher Education Policy.

Hassouneh, D., Lutz, K. F., Beckett, A. K., Junkins, E. P., \& Horton, L. L. (2014). The experiences of underrepresented minority faculty in schools of medicine. Medical Education Online, 19, 10.3402/meo.v19.24768. http://doi.org/10.3402/meo.v19.24768 
Hehman, E., Mania, E. W., \& Gaertner, S. L. (2010). Where the division lies: Common ingroup identity moderates the cross-race facial-recognition effect. Journal of Experimental Social Psychology, 46(2), 445-448.

Hewege, C. R. (2011). Acculturation and Management Control-'Japanese Soul in Sri Lankan Physique'. Contemporary Management Research, 7(1).

Hochschild, Russell, A., (1983), The Managed Heart. Commercialization of Human Feeling, L.A., University of California Press.

Hirshfield, L. E., \& Joseph, T. D. (2011) 'Why don't you get somebody new to do it?' Race and cultural taxation in the academy, Ethnic and Racial Studies, 34:1, 121141,DOI:10.1080/01419870.2010.496489

Hurtado, S., Clayton-Pedersen, A., Allen, W., \& Milem, J.. (1998). Enhancing Campus Climates for Racial/Ethnic Diversity: Educational Policy and Practice. Review of Higher Education, 21(3), 279 302.https://doiorg.ezproxy.fiu.edu/10.1353/ rhe. 1998.0003

Jacques, R. (1997). The unbearable Whiteness of being: Reflections of a place, stale male. In P.Prasad, A. J. Mills, M. Elmes, \& A. Prasad (Eds.), Managing the organizational melting pot: Dilemmas of workplace diversity (pp. 80-106). Thousand Oaks, CA: Sage.

Johnson-Bailey, J., \& Cervero, R. M. (2002). Cross-Cultural Mentoring as a Context for Learning. New Directions For Adult \& Continuing Education, 2002(96), 15.

Johnsrud, Linda K., and Kathleen C. Sadao. 1998. "The Common Experience of 'Otherness: Ethnic and Racial Minority Faculty.” Review of Higher Education 21 (4): $315-42$

June, AW. (2016). The invisible labor of minority professors. The Chronical of Higher Education. 11-15.

Kelley, H. (1973). The process of causal attribution. American Psychologist. 28, $107-128$.

Kolade, F. The Lived Experience of Minority Nursing Faculty: A Phenomenological Study. Journal of Professional Nursing, Volume 32, Issue 2, 2016, Pages 107-114, ISSN 8755-7223, https://doi.org/10.1016/j.profnurs.2015.09.002. (http://www.sciencedirect.com/science/article/pii/S8755722315001155)

Konrad, A. M. (2005). Examining the contours of workplace diversity. Handbook of workplace diversity, 1. 
Krashen, S.D. (1982). Accounting for child-adult differences in second language rate and attainment. Child-adult differences in second language acquisition ( $p$ .202-226). Rowley, MA: Newbury House.

Kundu, S. C. (2001). Managing cross cultural diversity. Delhi Business Review, 2(2).

Kvale, S. (n.d.). Dominance through interviews and dialogues. Qualitative inquiry, 12(3), 480-500. https://doi-org.ezproxy.fiu.edu/10.1177/1077800406286235

Laden, B. V., \& Hagedorn, L. S. (2000). Job satisfaction among faculty of color in academe: Individual survivors or institutional transformers?. New directions for institutional research, 2000(105), 57-66.

Laden, B. (2004) Hispanic-Seerving Institutions: What are they? Where are they?, Community College Journal of Research and Practice, 28:3, 181-198, DOI: $10.1080 / 10668920490256381$

Lauring, J., \& Selmer, J. (2011). Multicultural organizations: common language, knowledge sharing and performance. Personnel Review, 40(3), 324-343.

Lechuga, V. M. (2012). Emotional Management and Motivation: A Case Study of Underrepresented Faculty. New Directions for Institutional Research, (155), 8598. Retrieved from http://ezproxy.fiu.edu/login?url=http://search.ebscohost .com/login.aspx?direct=tru\&db=eric\&AN=EJ984270\&site=eds-live

Li, X. (2007). Characteristics of minority-serving institutions and minority undergraduates enrolled in these institutions (NCES 2008-156). Washington, DC: National Center for Education Statistics, Institute of Education Sciences, U.S. Department of Education.

Lincoln, Y. \& Guba, E. (1985). Naturalistic inquiry. Beverly Hills, CA: Sage.

Lincoln, Y.S., Lynham, S.A., \& Guba, E.G. (2011). Paradigmatic controversies, contradictions, and emerging confluences, revisited. In N.K. Denzin \& Y.S. Lincoln (Eds.). The SAGE handbook of qualitative research (pp. 97 - 128). Thousand Oaks, CA: SAGE

Lucas, C. J. (2006). American higher education: A history. New York: Palgrave Macmillan.

Mahoney, M. R., Wilson, E., Odom, K. L., Flowers, L., \& Adler, S. R. (2008). Minority Faculty Voices on Diversity in Academic Medicine: Perspectives From One School. Academic Medicine : Journal of the Association of American Medical Colleges, 83(8), 781-786.http://doi.org/10.1097/ACM.0b013e31817ec002https ://www.ncbi.nlm.nih.gov/pmc/articles/PMC2868964/ 
Marschan-Piekkari, R., Welch, D., \& Welch, L. (1999). In the shadow: The impact of language on structure, power and communication in the multinational. International Business Review, 8(4), 421-440.

Martin, G. C. (2014). The effects of cultural diversity in the workplace. Journal of Diversity Management (JDM), 9(2), 89-92.

Maslach C, Goldberg J. 1998. Prevention of burnout: new perspectives. Appl. Prev. Psychol. 7:63-74

Maslach, C., Schaufeli, W. B., \& Leiter, M. P. (2001). Job burnout. Annual review of psychology, 52(1), 397-422.

McKinley, B. (2003). The implementation of diversity in predominantly White colleges and universities. Journal of Black Studies, 34(1), 72-86.

Meyers, B. (2016). Where Are the Minority Professors?. The Chronical of Higher Education.

Minow, M. (1990). Making all the difference: Inclusion, exclusion, and American law. Ithaca: Cornell University Press.

Moustakas, C. E. (1994). Phenomenological research methods. Thousand Oaks, Calif. : Sage, c1994. Retrieved from http://http://search.ebscohost.com/login.aspx?direct= true $\& d b=$ cat $06026 a \& A N=$ fiu. $020249033 \&$ site $=$ eds-live

Niu, S., \& Tienda, M. (2013). High school economic composition and college persistence. Research in higher education, 54(1), 30-62.

Ogbonna E and Harris LC (2006) The dynamics of employee relationships in a diverse workforce. Human Relations 59(3): 379-407.

O’Reilly III, C. A., Caldwell, D. F., \& Barnett, W. P. (1989). Work group demography, social integration, and turnover. Administrative science quarterly, 21-37.

Padilla, A. M. (1994). Ethnic minority scholars, research, and mentoring: Current and future issues. Educational Researcher, 23(4), 24-27.

Peters, B. J., Overall, N. C., \& Jamieson, J. P. (2014). Physiological and cognitive consequences of suppressing and expressing emotion in dyadic interactions. International Journal of Psychophysiology, 94(1), 100-107. http://doi.org/10.1016/j.ijpsycho.2014.07.015

Pezdek, K., Blandon-Gitlin, I., \& Moore, C. (2003). Children's face recognition memory: More evidence for the cross-race effect. Journal of Applied Psychology, 88(4), 760 . 
Pololi, L., Cooper, L. A., \& Carr, P. (2010). Race, disadvantage and faculty experiences in academic medicine. Journal of General Internal Medicine, 25(12), 1363-1369.

Poveda, S. \& Roberts, T.. (2017) Critical agency and development: applying Freire and Sen to ICT4D in Zambia and Brazil, Information Technology for Development, 24:1, 119-137, DOI: 10.1080/02681102.2017.1328656

Rodríguez, J. E., Campbell, K. M., \& Pololi, L. H. (2015). Addressing disparities in academic medicine: what of the minority tax? BMC Medical Education, 1(15), 6.

Said, E. W. (2012). Culture and imperialism. Vintage.

Salinas C. \& Lozano, A. (2017) Mapping and recontextualizing the evolution of the term Latinx: An environmental scanning in higher education, Journal of Latinos and Education, DOI: 10.1080/15348431.2017.1390464

Saldaña, J. (2009). The coding manual for qualitative researchers. London; Thousand Oaks, Calif. : Sage, 2009. Retrieved from http://http://search.ebscohost.com/ login.aspx?direct $=$ true $\& \mathrm{db}=$ cat $06026 \mathrm{a} \& \mathrm{AN}=$ fiu $.020334783 \&$ site $=$ eds-live

Sandelowski, M. (1995), "Sample size in qualitative research," Research in nursing \& health, Vol. 18 No. 2, pp. 179-183

Scarborough, W. J., Lambouths III, D. L., \& Holbrook, A. L. (2019). Support of workplace diversity policies: The role of race, gender, and beliefs about inequality. Social Science Research, 79, 194-210. https://doi.org/10.1016/j.ssresearch.2019.01.002

Schaefer, R. T. (2012). Racial and ethnic groups (13th ed.). Upper Saddle River, NJ: Pearson Prentice Hall.

Seidman, I. (2013). Interviewing as qualitative research : a guide for researchers in education and the social sciences. New York: Teachers College Press, c2013.

Shavelson, R. J., Hubner, J. J., \& Stanton, G. C. (1976). Self-concept: Validation of construct interpretations. Review of educational research, 46(3), 407-441.

Shavers, M. C., Butler, J., \& Moore, III, J. L. (2014). Cultural taxation and the over commitment of service at predominantly White institutions. In F. A. Bonner et al. (Eds.), Black faculty in the academy: Narratives for negotiating identity and achieving career success. 41-52. New York: Routledge.

Simon, M.K. (2011). Assumptions, limitations and delimitations. From Dissertation and scholarly research: Recipes for success. Seattle, WA: Dissertation Success, LLC. 
Skidmore, T. E., Smith, P. H., \& Green, J. N. (2010). Modern Latin America (7th ed.). Oxford, UK: Oxford University Press.

Smith, D. G. (1989). The Challenge of Diversity. Involvement or Alienation in the Academy? ASHE-ERIC Higher Education Report No. 5, 1989. ASHE-ERIC Higher Education Reports, The George Washington University, One Dupont Circle, Suite 630, Dept. ES, Washington, DC 20036-1181.

Snyder, M. (1982). "Self-fulling Stereotypes." In Rothenburg, P. (Ed.). Race, class, and gender in the United States - An integrated study (10th ed.) 541-546. New York: Worth Publishers.

Sporer, S. L. (2001). The cross-race effect: Beyond recognition of faces in the laboratory. Psychology, Public Policy, and Law, 7(1), 170.

Stahl, G. K., Maznevski, M. L., Voigt, A., \& Jonsen, K. (2010). Unraveling the effects of cultural diversity in teams: A meta-analysis of research on multicultural work groups. Journal of international business studies, 41(4), 690-709.

Stalin, J. V. (2008). Marxism and problems of linguistics. Wildside Press LLC.

Stanley, C. A. 2006. "Coloring the Academic Landscape: Faculty of Color Breaking the Silence in Predominantly White Colleges and Universities." American Educational Research Journal 43 (4): 701-36

Stockdill, B. \& Danico, M. (2012). Transforming the ivory tower: Challenging racism, sexism, and homophobia in the academy.

Syed, M., Santos, C., Yoo, H. C., \& Juang, L. P. (n.d.). Invisibility of Racial/Ethnic Minorities in Developmental Science: Implications for Research and Institutional Practices. AMERICAN PSYCHOLOGIST, 73(6), 812-826. https://doi.org/10.1037/amp0000294

Trower, C. A. (2008). Amending higher education's constitution. Academe, 94(5), 1618,3. Retrieved from http://ezproxy.fiu.edu/login?url=https://search.proquest.com/ docview/232296149?accountid=10901

Trower, C. (2003). Leveling the field. The Academic Workplace, 14(2), 1-15.

Turner, C. V., \& Gonzalez, J. C. (2008). Faculty of Color in Academe: What 20 Years of Literature Tells Us. Journal Of Diversity In Higher Education, 1(3), 139-168. 
Utsey, S. O., Ponterotto, J. G., Reynolds, A. L., \& Cancelli, A. A. (2000). Racial discrimination, coping, life satisfaction, and self-esteem among African Americans. Journal of counseling and development, winter 2000(78), 72-80.

Vaismoradi, M., Jones, J., Turunen, H., \& Snelgrove, S. (2016). Theme development in qualitative content analysis and thematic analysis.

Van Laer, K., \& Janssens, M. (2011). Ethnic minority professionals' experiences with subtle discrimination in the workplace. Human Relations, 64(9), 1203-1227.

Van Manen, M. (2016). Phenomenology of practice : meaning-giving methods in phenomenological research and writing. London : Routledge, 2016. Retrieved from http://http://search.ebscohost.com/login.aspx?direct=true \&db=cat06026 a\&AN=fiu.003764356\&site=eds-live

Weiss, R. S. (1994). Learning from strangers : the art and method of qualitative interview studies. New York : Free Press; Toronto : Maxwell Macmillan Canada; New York: Maxwell Macmillan International.

Wijesingha, R., \& Ramos, H. (2017). Human capital or cultural taxation: What accounts for differences in tenure and promotion of racialized and female faculty?. Canadian Journal of Higher Education/Revue canadienne d'enseignement supérieur, 47(3), 54-75.

Young, I. M. (2013). Five faces of oppression. In The community development reader (pp. 346-355). Routledge.

Young, Iris M. (2014). The five faces of oppression. In Rethinking power, ed. T. Wartenberg. (pp. 1-22) Albany: SUNY Press.

https://nces.ed.gov/fastfacts/display.asp?id=61 


\section{Appendix A}

\section{Qualitative Interview Study Research Question:}

To what extent are the experiences of faculty and administrators related to cultural taxation?

\section{Interview Questions}

1. How do you identify racially and ethnically?

2. Describe your experience as an underrepresented minority at FIU?

a. What were your initial feelings about the environment?

3. Can you tell me about anything you felt you have had to do, or were expected to do outside of your job duties because of your cultural, racial, or ethnic difference (Cultural Taxation)?

a. Critical Agency

i. Do you feel like you have different responsibilities based on your racial or ethnic background?

1. What do you perceive are some positive and or negative impacts of these responsibilities?

b. Expectations

a. Why did you didn't you complete these acts?

i. Tell me about (Internal and/or external) expectations as a member of the underrepresented community?

1. Can you give me an example?

a. How do you feel about these acts and/or expectations?

c. Coping with problematic colleagues' behavior

i. What (positively and negatively) impacts your relationship with your colleagues?

1. Can you give me an example of either or both?

d. Differential legitimacy

i. Can you tell me about a time you felt as though you had to prove yourself or abilities because of your cultural, racial, or ethnic difference?

e. Acculturation

i. How did you navigate the campus culture as a new member of the community?

f. Over-commitment

i. Have you ever felt that you were overused or overly called on because of your racial or ethnic background?

g. Isolation

i. Do you feel like an integrated part of the community?

1. Why or why not?

a. How do you develop a sense of belonging?

h. Career progress 
i. Tell me about your career progress. What do you attribute it to? Why?

1. Do you feel as though being a member of an underrepresented group impacts your career progress?

ii. What role did or didn't mentoring or mentorship play as a part of your experience?

4. What challenges exist for underrepresented minority faculty and/or administration at FIU?

5. What factors contributed to your success at FIU?

6. What are your future aspirations within FIU?

7. What advice would you give any underrepresented minorities aspiring to work at FIU?

8. Are there any additional comments you wish to share regarding your experience at FIU 


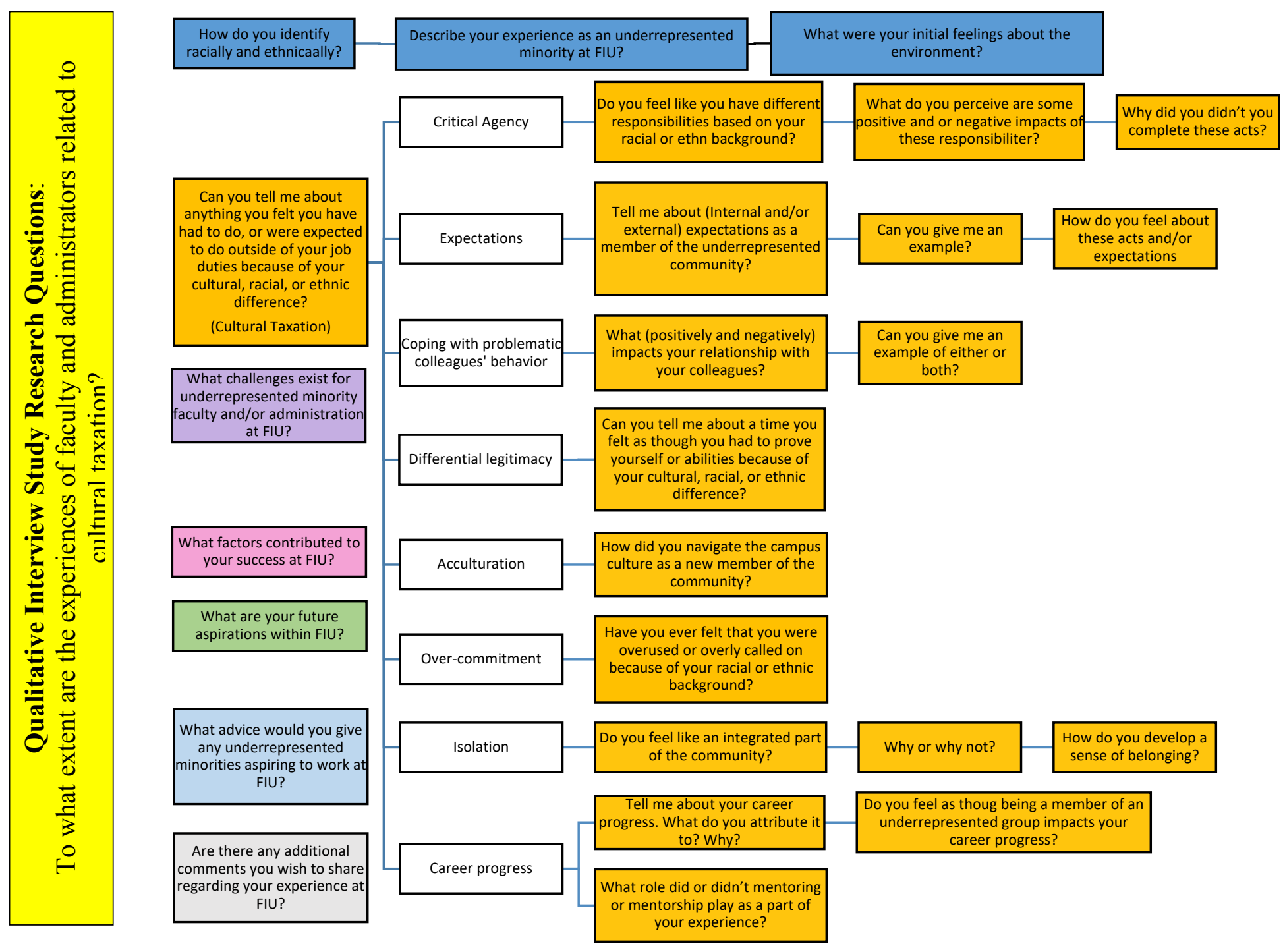




\section{Appendix B}

- Internal Imposition

\section{Cultural Taxation Coding Breakdown}

○ Obligation

- Taking responsibility for settling problems regarding sociocultural differences among the members of the institution

- Mentoring

- Stereotype Deconstruction

- Differential emotional management

- Coping Tactics

- Coping with colleagues' problematic behavior

- External Imposition

- Coping with students' problematic behaviors

o Service

- Serving on AA committees without any observable changes

- Serving as the liaison between the institution and the ethnic community even though one may not agree with the institutions' policies

- Being called upon to serve as a translator when the institution is presented with non-English speakers

- Mentoring

- Expectations

- Irregular Expectations

- Being called upon to serve as the "expert" on diversity matters

- Being called upon to educate members of the majority group about diversity

- Differential Legitimacy

- Social Imposition

○ Integration

- Acculturation

- Managing communication and language barriers

- Forced social integration 


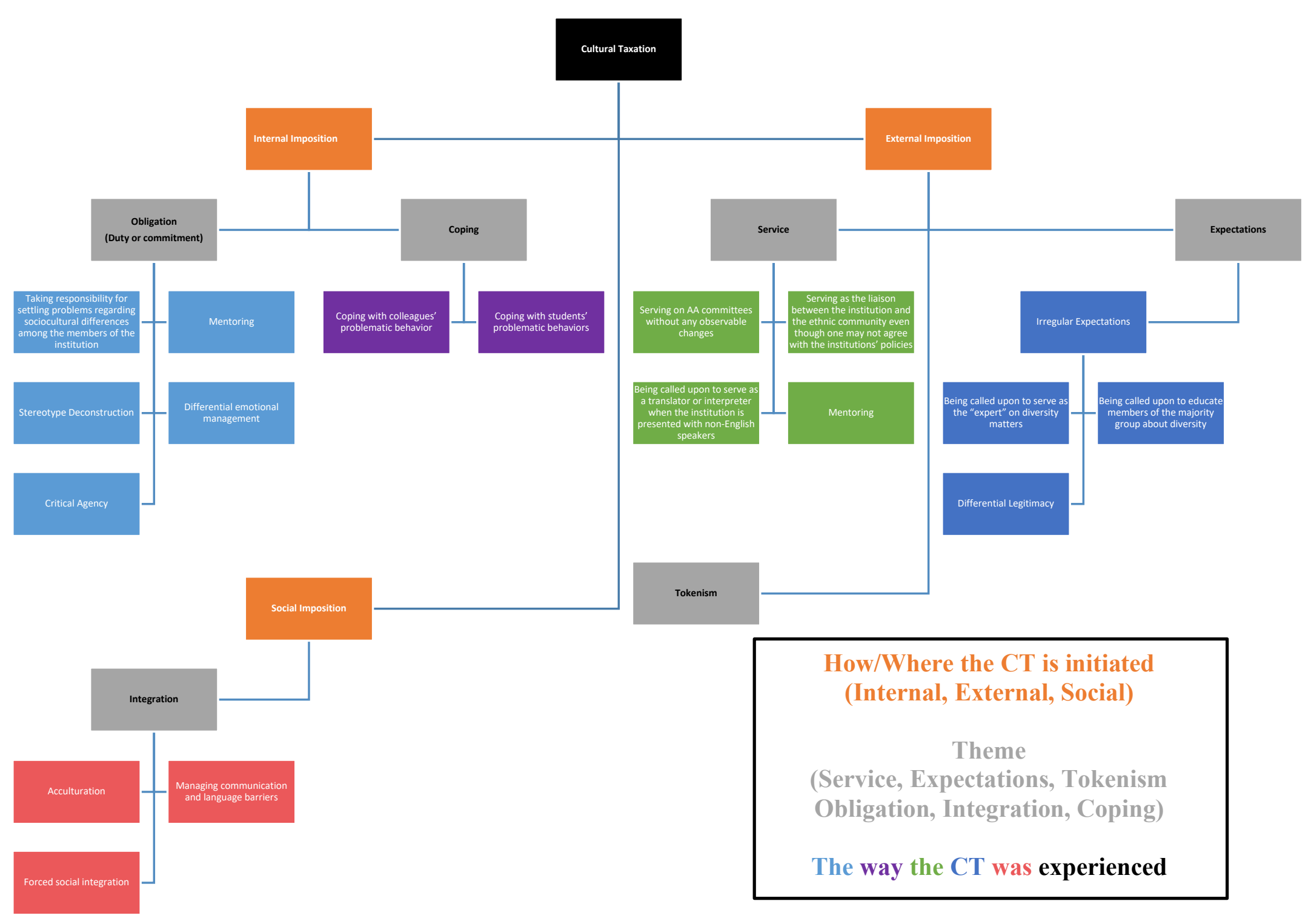


VITA

KATRINA NGOZI AMIE

2008-2010

2009-2010

2010-2011

2010-2012

2011-2014

2012-2014

2014-2015

2015 - Present
Associate of Arts, General Studies

Florida State University

Tallahassee, Florida

University Housing Conduct Board

Florida State University

Tallahassee, Florida

VP, SDES Student Advisory Council

Member

University of Central Florida

Orlando, FL

Bachelor of Science, Health Services

Administration

University of Central Florida

Orlando, Florida

Federal S.C.E.P (Pathways) Intern

Orlando V.A. Medical Center

Orlando, Florida

Master of Arts, Educational Leadership

University of Central Florida

Orlando, Florida

Program Assistant - Data Management

Orlando V.A. Medical Center

Orlando, Florida

Coordinator, Academic Support Services Florida International University

Miami, Florida 